\title{
WestVirginiaUniversity
}

THE RESEARCH REPOSITORY @ WVU

Graduate Theses, Dissertations, and Problem Reports

2018

\section{Satellite Based Predictability Of Water Sensitive Infectious Diseases}

Md. Rakibul Hassan Khan

Follow this and additional works at: https://researchrepository.wvu.edu/etd

\section{Recommended Citation}

Khan, Md. Rakibul Hassan, "Satellite Based Predictability Of Water Sensitive Infectious Diseases" (2018). Graduate Theses, Dissertations, and Problem Reports. 7196.

https://researchrepository.wvu.edu/etd/7196

This Dissertation is protected by copyright and/or related rights. It has been brought to you by the The Research Repository @ WVU with permission from the rights-holder(s). You are free to use this Dissertation in any way that is permitted by the copyright and related rights legislation that applies to your use. For other uses you must obtain permission from the rights-holder(s) directly, unless additional rights are indicated by a Creative Commons license in the record and/ or on the work itself. This Dissertation has been accepted for inclusion in WVU Graduate Theses, Dissertations, and Problem Reports collection by an authorized administrator of The Research Repository @ WVU.

For more information, please contact researchrepository@mail.wvu.edu. 


\title{
SATELLITE BASED PREDICTABILITY OF WATER SENSITIVE INFECTIOUS DISEASES
}

\author{
Md. Rakibul Hassan Khan
}

A DISSERTATION

Submitted to the Benjamin M. Statler College of Engineering and Mineral Resources at West Virginia University in partial fulfillment of the requirements for the

Degree of Doctor of Philosophy in

Civil and Environmental Engineering

Antarpreet Jutla, PhD, Chair

Andrew Monaghan, PhD

Avinash Unnikrishnan, $\mathrm{PhD}$

Lian-Shin Lin, PhD

Radhey Sharma, PhD

\section{Department of Civil and Environmental Engineering}

\section{Morgantown, West Virginia}

2018

Keywords: Water Related Diseases, Cholera, Vibrio, Natural Disaster, Predictability, Satellite, Remote Sensing, Risk, Precipitation, Seasonality

Copyright 2018 Md. Rakibul Hassan Khan 


\section{ABSTRACT \\ SATELLITE BASED PREDICTABILITY OF WATER SENSITIVE INFECTIOUS DISEASES}

\section{Md. Rakibul Hassan Khan}

The global human population remains at heightened risk of diarrheal diseases after natural disasters, such as hurricanes, earthquakes, floods or droughts. The uncertainties in timing and magnitude of natural disasters impact the hydroclimatic baseline, and/or access to safe drinking water and sanitation infrastructure (WASH). Also, data on disease prevalence and infectious pathogens is sparingly available in the region(s) where climatic variability and extreme natural events intersect with population vulnerability. Therefore, traditional time series modeling approach of calibration and validation of a model is inadequate and predictions of diarrheal infections remain a challenge. From this context, it is pivotal to understand the role of hydroclimatic processes in creating seasonality and inter-annual variability in environmental conditions favorable for exposure to pathogenic agents (e.g. bacteria) that lead to outbreaks of environmentally modulated water-related diseases. Here, using cholera as one of the signature diarrheal diseases, a framework is proposed that can be used to assess the impact of natural disasters with response to an outbreak of cholera, providing an assessment of short-term and longterm influence of climatic processes on disease outbreaks is human. Cholera, a deadly waterborne diarrheal disease is transmitted by drinking water contaminated with Vibrio cholerae, an autochthonous bacterium. Prediction of cholera, using earth observations, especially for regions where hydroclimatic and disease surveillance data are not routinely collected, is a critical tool to prioritize prevention and mitigation strategies, such as the distribution of oral rehydration solutions, strengthening WASH infrastructure, and increasing the availability of antibiotics and 
vaccines. A new algorithm was developed that integrates satellite derived data on several hydroclimatic and ecological processes into a framework that can determine high resolution cholera risk on global scales. Using satellite-derived hydroclimatic data and information on WASH, the algorithm tracks the changing environmental conditions conducive to the growth of pathogenic vibrios. The algorithm was applied following hurricane Matthew in 2016 in Haiti, two consecutive earthquakes in 2015 in Nepal and recent civil unrest in Yemen with realistic accuracy in forecasting the risk of a cholera outbreak in the human population. A software version (KJ Cholera Forecast, V 1.0) of the algorithm with a user-friendly graphical user interface (GUI) was also developed to generate near real time cholera forecast on a global scale. Also, the abundance, distribution and environmental linkages of vibrio species were explored and modeled to understand the potential risk of emergence in the coast. Prediction systems when incorporated with vaccine protocols and long term strategies for development of civil infrastructure, can provide the capacity needed to eradicate the burden of cholera in a human population, if not the disease itself. 


\section{DEDICATION}

I dedicate this dissertation to my daughter Tazmeen Khan, the greatest inspiration of my life. 


\section{ACKNOWLEDGEMENTS}

This research was funded by NASA Health and Air Quality program and NSF. I gratefully acknowledge the guidance, encouragement and support of Dr. Antar Jutla, my thesis advisor. I am also grateful to him for his insightful comments, suggestions and definitely his intellectual support.

I would also like to thank Dr. Andrew Monaghan, Dr. Avinash Unnikrishnan, Dr. Lian-Shin Lin and Dr. Radhey Sharma, for serving on my $\mathrm{PhD}$ committee and addressing specific concerns, providing suggestions and assistance regarding my dissertation. I would specially thank Dr. Andrew Monaghan for providing me the opportunity to work as an ASP doctoral fellow at the National Center for Atmospheric Research during my $\mathrm{PhD}$ and gain valuable research experience. I would like to take the opportunity to thank Dr. Fariborz Nasr, Haidar Aaldach, Moiz Usmani, Rifat Anwar and Ashish Kondal for their valuable time and interest in my research.

I extend my deep appreciation to my wife, Tania Rahman and parents, Md. Sharafat Ali Khan \& Rahima Khanam for their unconditional moral support and encouragement. 


\section{TABLE OF CONTENT}

CHAPTER 1: INTRODUCTION. .1

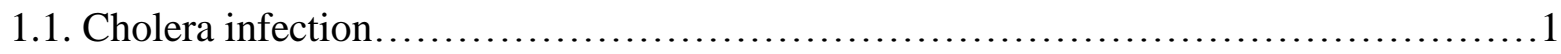

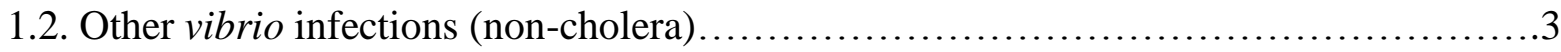

1.3. Natural disaster and cholera epidemics......................................... 4

1.4. Remote sensing as emerging technology for predictability of disease outbreak............6

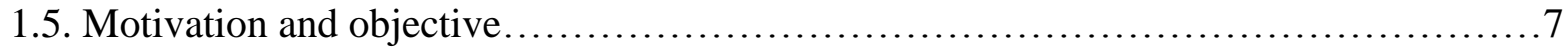

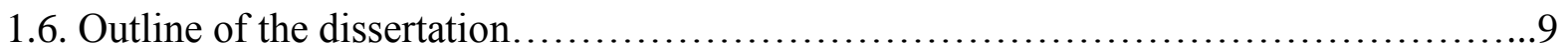

CHAPTER 2: NATURAL DISASTERS AND CHOLERA OUTBREAKS:

CURRENT UNDERSTANDING AND FUTURE OUTLOOK.................11

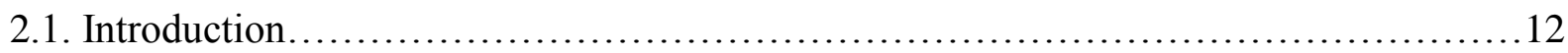

2.2. Natural disasters and cholera epidemics....................................... 15

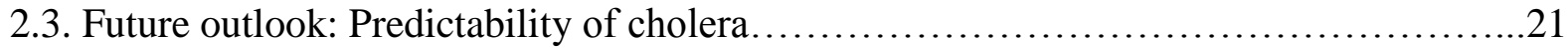

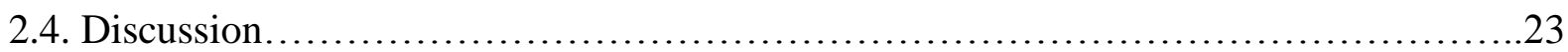

CHAPTER 03: ASSESSMENT OF RISK OF CHOLERA IN HAITI

FOLLOWING HURRICANE MATTHEW ................................26

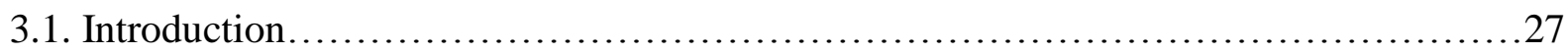

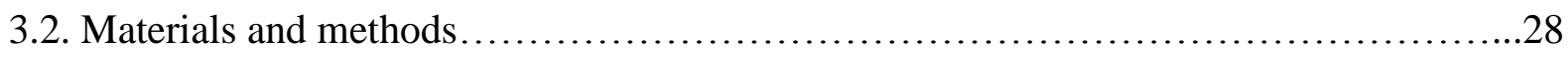

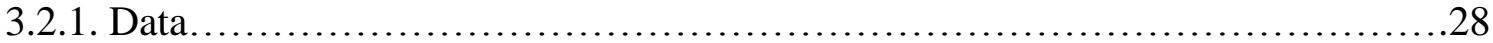

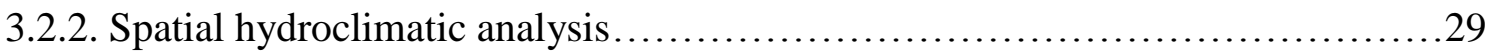

3.2.3. Composite weighted raster overlay for cholera risk mapping ....................30

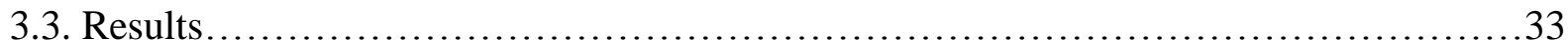

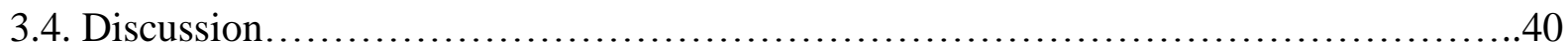

CHAPTER 04: EVALUATION OF RISK OF CHOLERA AFTER A NATURAL DISASTER: LESSONS LEARNED FROM THE 2015 NEPAL

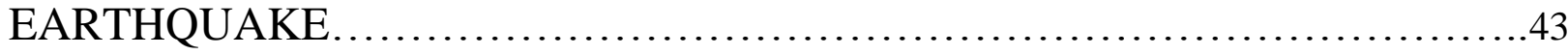

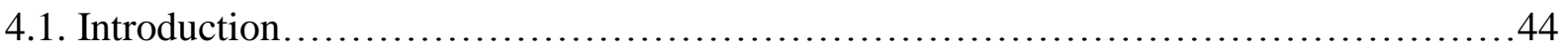




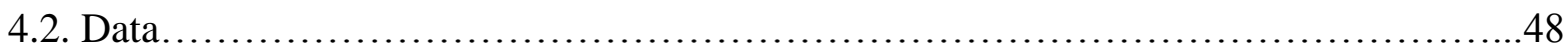

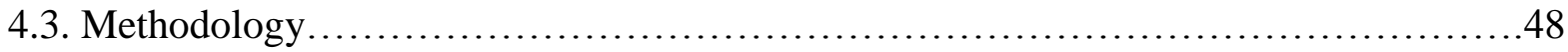

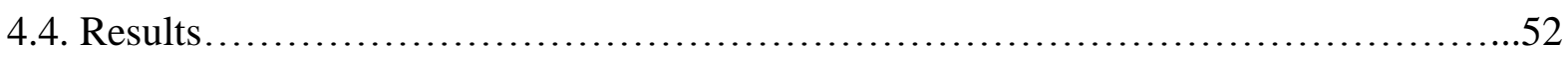

4.4.1. Hydroclimatological risk of cholera .....................................53

4.4.2. Integrated WASH and hydroclimatological risk...........................55

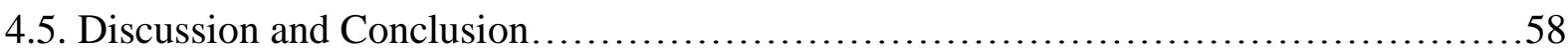

\section{CHAPTER 05: EXPLORATORY ANALYSIS OF PATHOGENIC VIBRIOS IN COASTAL WATER ........................................................61}

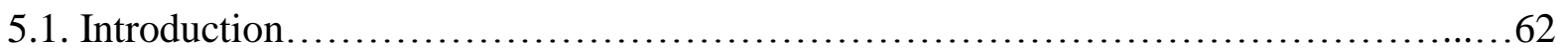

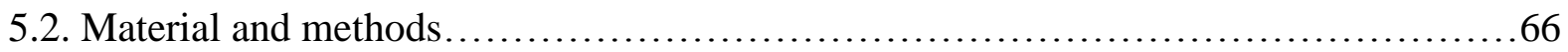

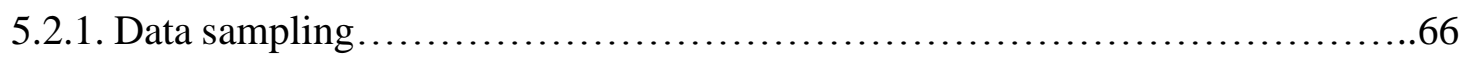

5.2 .2 . Water quality analysis ...................................................

5.2.3. Vibrio presence data..................................................68

5.2.4. Remote Sensing data used for coastal vibrio niches.........................69

5.2.5. Statistical exploratory analysis methods ................................. 70

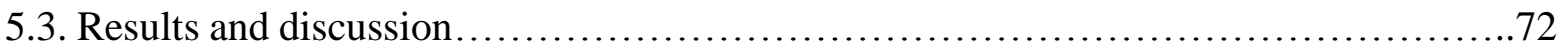

5.3.1. Distribution and abundance of selected Vibrio genes across samples...........72

5.3.2. Seasonality of Vibrio genes............................................. 74

5.3.3. Variability of Vibrio seasonality across samples, genes and locations...........76

5.3.4. Ecological linkages of Vibrio spp. and emergence modeling ...................77

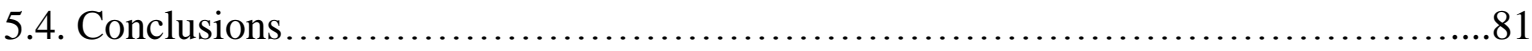

CHAPTER 6: CONCLUSION ........................................... 83

6.1. Towards an operational near real time cholera forecasting system...................83

6.2. Development of cholera risk forecasting software (Khan-Jutla Cholera Modeling

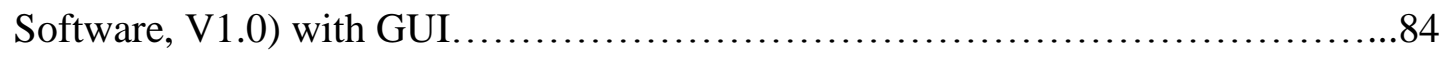

6.3. Operational near real time cholera forecasting in Yemen: An application..............87

6.4. Conclusion and key contributions............................................ 94 


\section{LIST OF TABLES}

Table 2.1: Common effects of natural disasters on societal services (Source: PAHO)...........17

Table 2.2: Diarrheal diseases modeling around the globe using remotely sensed data...........23

Table 2.3: Results of web search using SCOPUS on cholera and several related keywords in

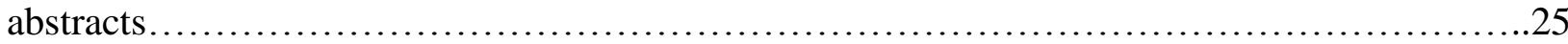

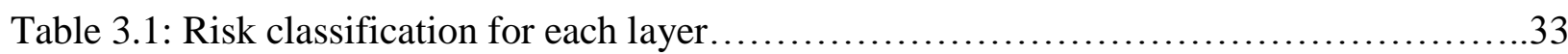

Table 4.1: Hydro-climatological risk weights used in cholera outbreak risk clustering..........50

Table 4.2: Socio-hydro-climatological risk weights used in cholera outbreak risk

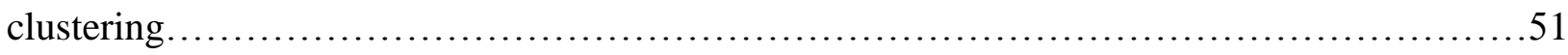

Table 5.1: Data used for coastal vibrio niche modeling................................70

\section{LIST OF FIGURES}

Figure 1.1: Cholera hotspot globally based on prevalence data from 1950 to 2015 (Source:

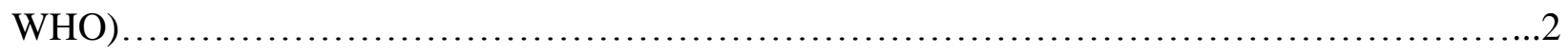

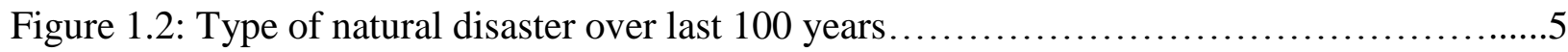

Figure 1.3: Remote sensing aided framework for predictability of water related infectious

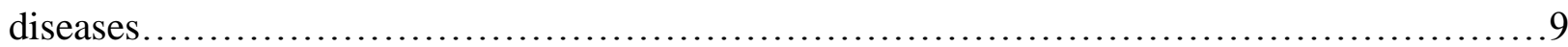

Figure 2.1: Cholera map showing number of years of reported cholera patients in WHO

database....

Figure 2.2: Framework to understand environmental pathways for an outbreak of cholera after

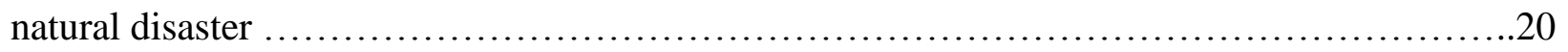

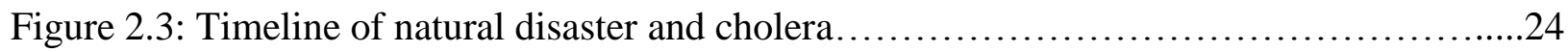


Figure 3.1: Epidemic cholera risk hypothesis

Figure 3.2: Composite weighted raster overlay algorithm.

Figure 3.3: Hurricane Matthew tracking over Haiti.

Figure 3.4a: August air temperature anomalies

Figure 3.4b: September air temperature anomalies................................... 34

Figure 3.5a: September (1st through 30th) precipitation anomalies.........................35

Figure 3.5b: (Sept7-Oct6) precipitation anomalies.................................. 35

Figure 3.5c: (Sept14-Oct13) precipitation anomalies................................... 36

Figure 3.6a: Cholera risk map based on pre hurricane hydro-climatic conditions for the month of

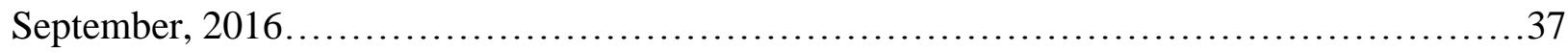

Figure 3.6b: Cholera risk map for the post hurricane period October 07 to November 06, 2016.

Figure 3.6c: Cholera risk map for the post hurricane period October 14 to November 13, 2016

Figure 3.7: Cholera in Haiti up to October 10, 2016

Figure 3.8: Predictive risk map for cholera post hurricane period November 01 to November 30 .

Figure 4.1: Weighted raster overlay, with blue dotted box representing layers used to generate the hydroclimatic risks map and solid red box incorporating water resources into the risk calculations.

Figure 4.2: Hydroclimatological anomalies for May through August, 2015. .53 
Figure 4.3. Cholera risk map based on hydroclimatology of the region.

Figure 4.4. Status of water resources (a) sanitation accessibility and (b) safe water accessibility in Nepal .55

Figure 4.5. Cholera risk map post-earthquake with resilient (present or intact) water resources and hydroclimatology .56

Figure 4.6. Cholera risk map post-earthquake including a devastated water supply after the earthquake (circles show extent of the earthquake).

Figure 5.1. Map of Chesapeake Bay showing sampling sites in the Chester River and Tangier

Sound. .68

Figure 5.2. Violin plot of vibrio genes detected in water, oyster and sediment samples....

Figure 5.3. Ternary densities of Vibrio detected in water, oyster, and sediment .74

Figure 5.4. Seasonality of Vibrio genes detected in water, oyster, and sediment samples.... .75

Figure 5.5. Ecological niches of Vibrio genes detected in water, oyster, and sediment samples .79

Figure 5.6: Emergence risk of V.cholerae based on ensemble model.

Figure 6.1: Khan-Jutla Cholera Modeling Software (Version 1.0)........................86

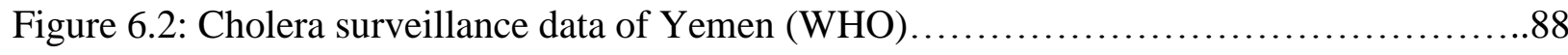

Figure 6.3: Cholera risk map for October 2016. .88 
Figure 6.4: Cholera risk prediction in Yemen with one month lead time: a) April cholera risk prediction b) May cholera risk prediction c) June cholera risk prediction d) June cholera

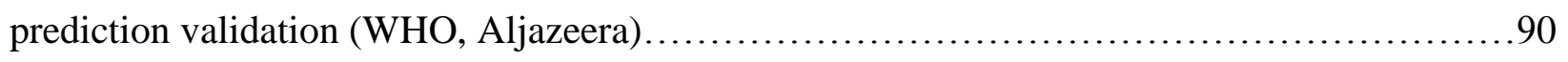

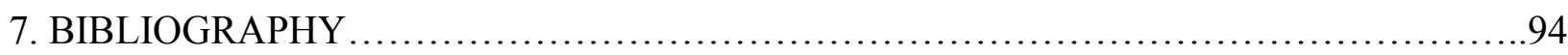




\section{CHAPTER 1: INTRODUCTION}

\subsection{Cholera}

Water-related diarrheal diseases, such as cholera, remain second leading cause of child death worldwide, killing over 1.5 million children annually and infecting millions more (Bartram, 2008). Linking satellite based earth observations with ecology of vibrios remains a challenge, primarily due to spatio-temporal scale mismatch that further limits development of predictive algorithms for understanding physical pathways of disease trigger and transmission in human population. For most waterborne pathogens, surveillance is spotty, diagnoses are not uniform, and understanding of the effects of climate related drivers remains limited. Diarrheal diseases are unlikely to be eradicated (Figure 1.1) since the disease causing agents are always present and adapt to environment (Constantin de Magny et al., 2008). Cholera, the focus disease of this dissertation, occurs in two major forms-epidemic (sudden outbreaks) and endemic (recurrence and persistence of the disease for several consecutive years) with significant spatial and temporal heterogeneity. While significant research on hydroclimatic drivers (trigger of conditions) and cholera is available in literature (Jutla et al., 2013), yet we are not able to predict how transmission of this disease occurs in human population?

Cholera transmits via drinking water contaminated with Vibrio cholerae bacteria, which is the main causative agent of cholera (Bentivoglio and Pacini, 1995). For an epidemic cholera outbreak to occur in a region, broadly three criterions are required to be fulfilled: 1) presence of $V$. cholerae in waterbodies, the causative agent of cholera, near to high population settlement 2) triggering hydroclimatic condition for the growth and proliferation of vibrios, 3) a fragile or broken water and sanitation accessibility or in other words WASH infrastructure for the transmission of 
vibrios to human population (Khan et al., 2017, Jutla et al., 2013). V. cholerae is known for its ability to cause pandemics, thereby it is considered as unique among the diarrheal pathogens (Austin, 2010 and Faruque et al., 2004). There are around 200 serogroups of this bacteria denoted by $\mathrm{O} 1, \mathrm{O} 2$, etc. Two strains of cholera are associated with the infection: O1 and O139; however, some other strains can cause diarrhea (Penrose et al., 2010; Faruque et al., 2004). The epidemic form of cholera can be defined as the sudden occurrence of cholera at a particular time and place. However, if the disease occurs frequently in a region for a period of at least 10 years, a region may be defined as an endemic one.

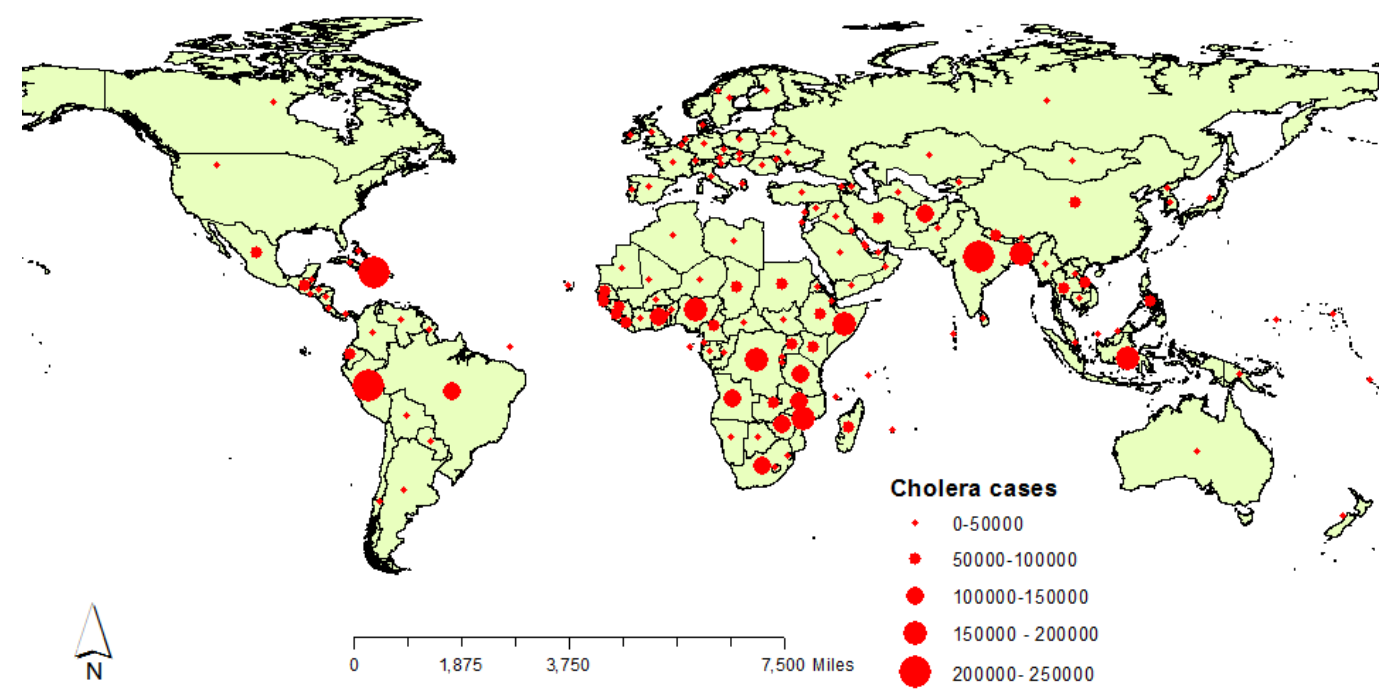

Figure 1.1: Global cholera hotspots based on prevalence data from 1950 to 2015 (Data: WHO)

With limited efficacy of cholera vaccines and the health threat posed by this disease, it is very important to understand the disease dynamics with environment and society (Emch et al., 2008). Changes in intensity and seasonality of hydro-climatological processes is likely to affect ecological niches of vibrios that may result in disease outbreak in new regions. In addition, natural disasters, such as earthquake, flooding or droughts that may expose human population to natural 
pathways of bacteria. Once humans are exposed to infective doses of cholera bacteria, transmission of disease occurs via shedding of pathogens in water systems, leading to an outbreak.

The growth of $V$. cholera is hypothesized to be related to episodic variability in various eco-hydrological processes such as precipitation (Hashizume et al., 2008), floods (Koelle et al., 2005), peak river level (Schwartz et al., 2006), sea surface temperature (SST) (Lobitz et al., 2000; Pascual, 2000) and sea height (Lobitz et al., 2000), water temperature (Huq et al., 2005), plankton abundance (Worden et al., 2006; Jutla et al., 2013) and fecal contamination (Islam et al., 2009). Changes in intensity and seasonality of hydrological processes is likely to affect ecological niches of vibrios, which may result in disease outbreak in new regions. In addition, acceleration in hydrological cycles may lead to natural disasters, such as flooding or droughts that may expose human population to natural pathways of bacteria. Once humans are exposed to infective doses of cholera bacteria, transmission of disease occurs via shedding of pathogens in water systems, leading to an outbreak.

\subsection{Other vibrio infections (non-cholera)}

Vibrio vulnificus and Vibrio parahaemolyticus are human pathogens that are responsible for thousands water-borne cases in the United States each year. Vibrio spp. are autochthonous in marine and estuarine environments, and, therefore the abundance and distribution of Vibrio spp. are critical for disease prevention. Vibrio pathogens have long been established as native to the aquatic environment and present in the water column, sediment, and in filter feeders, e.g., shellfish (Vezzulli et al., 2010; Colwell, 1996). The occurrence of Vibrio spp. in rivers, estuarine, coastal waters, and the deep sea is significantly influenced by p revailing environmental conditions and, therefore, can pose a significant public health threat to various countries and cultures. Both $V$. vulnificus and $V$. parahaemolyticus can cause mild to severe health conditions, including 
gastroenteritis and septicemia (Wright et al., 1996; Oliver, 1989). V. vulnificus can cause primary septicemia associated with raw oyster consumption and also severe wound infections when individuals are exposed to contaminated seafood or seawater. The mortality rate of $V$. vulnificus induced septicemia can exceed 50\% and death typically occurs within 48 hours. Mortality of individuals suffering $V$. vulnificus infected wounds is $25 \%$.

\subsection{Natural disaster and cholera epidemics}

Natural disasters jeopardize the societal structure (e.g. safe drinking water accessibility and adequate sanitation services) and thus indorse the spread of many serious water-borne diseases. Natural disasters such as earthquakes, hurricane, landslides, tsunamis, floods, drought etc. can prove catastrophic to surge water borne disease burden (Watson et al., 2007). Cholera, an acute diarrheal illness caused by infection of the intestine with $V$. cholerae and acquired by drinking water or eating food contaminated with the cholera bacterium can spread rapidly in areas with inadequate treatment of sanitation and drinking water (Ligon et al., 2006). In the days and weeks following a natural disaster, the threat of water borne disease outbreaks is extremely high.

Disrupted societal structures form a combination of stress in safe drinking water, inadequacy in sanitation services, enforced crowding of human population, and consequently propagate diarrheal disease outbreak condition via drinking water contaminated with bacteria. During a natural disaster, a large numbers of people are forced to seek temporary shelter in crowded conditions with inadequate sanitation access, improper waste management, compromised sources of water, and a low level of immunity, all factors that play a key role in compounding the devastation of an outbreak (Stephen et al., 2005). According to WHO, cholera continues to be a major global threat in many developing regions of the world, and the threat of an epidemic is constant throughout any given year. 
A natural disaster may occur at any time in any part of the world. The aftermath of natural disasters is no less serious than the immediate destruction they cause. After most catastrophes great numbers of people are left homeless, deprived of good sanitation services and safe drinking and consequently exposed to adverse climatic conditions and to the spread of disease (Lorna et al., 2005). Floods (Figure 1.2) are perhaps one of the largest subgroups amongst all the disasters (EM DAT, 2016). Overall, an increasing trend (Figure 1.2) can be observed in the total number of natural disasters post 1960, arising from enhanced anthropogenic activities e.g. rapid urbanization, deforestation, environmental degradation(Leaning and Guha-Sapir, 2013).

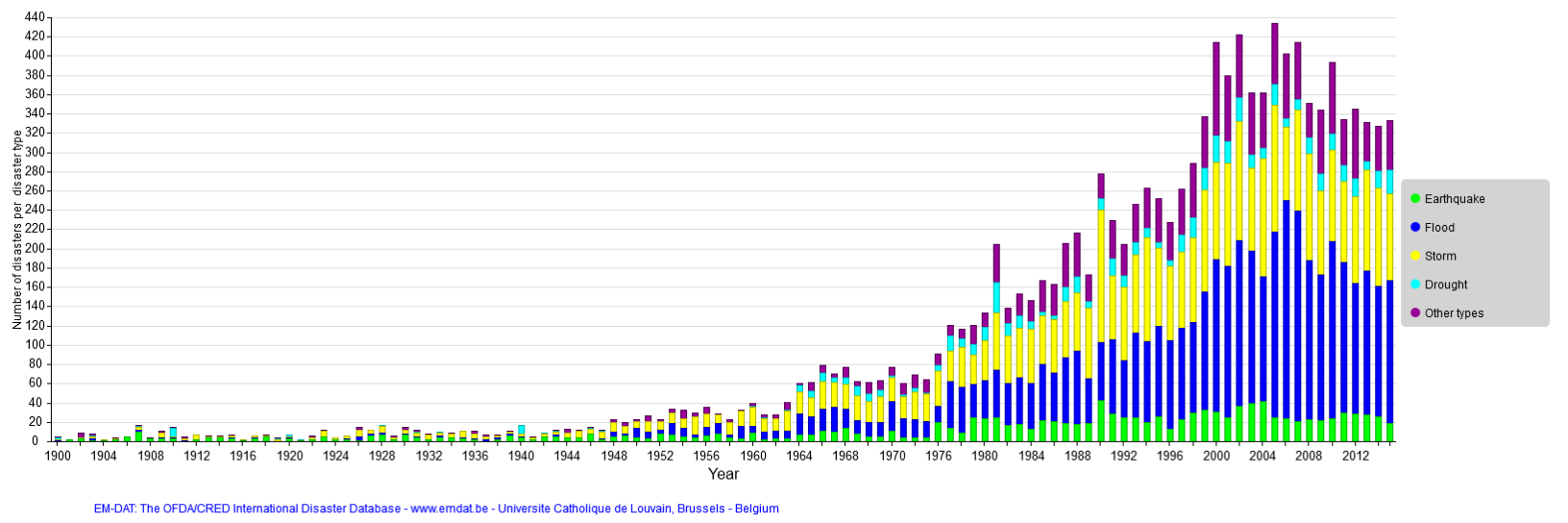

Figure 1.2: Type of natural disaster over last 100 years.

Rapid and sustained diarrheal transmission after introduction of the disease is not surprising (WHO, 2008). The earthquake in Pakistan resulted 23,405 cases of acute watery diarrhea in 2005 (Ligon et al., 2006). Diarrhea has been the leading cause of death among Haitian children $<5$ years of age and given the limited access to clean water and sanitation after 2010 earthquake. The lack of water and sanitation services caused the severe spread of the cholera epidemic in Haiti and resulted in 658,563 reported cases with 8,111 reported deaths from 2010 to 2013 (Gelting et al., 2013). The 2015 earthquakes in Nepal endangered the cholera outbreak as water and sanitation 
infrastructure were collapsed. Earthquakes have left around 1.1 million people without access to protected water sources. Significant damage to latrines has also left another 1.1 million people without safe, hygienic and dignified means to access sanitation facilities (UN, 2015). The deteriorated societal structures along with favorable hydro-climatic condition for bacterial growth

aggravated the spread of cholera in capital Katmandu. A large (>16,000 cases) cholera epidemic in West Bengal in 1998 was attributed to preceding floods (Sur et al., 2000). More than 350,000 cases of diarrhea resulted from the July 2004 flood in Bangladesh. In the United States, after the Katrina hurricane approximately 1000 cases of diarrhea and vomiting among adults and children were reported in Mississippi and Texas. Diarrheal illness was noted after Hurricanes Allison in Texas (Waring et al., 2002).

\subsection{Remote sensing as emerging technology for predictability of disease outbreak}

High resolution remote sensing data are becoming increasingly accurate through calibration with real-time information on location. Availability of high resolution and accurate satellite data provides tremendous promise for prediction of diarrheal diseases, weeks and even months in advance of an epidemic (Ford et al., 2009). Linking satellite based earth observations with ecology of bacteria is getting easier, primarily due to access of satellite data and thus understanding physical pathways of disease outbreak in human population. Increased computing power and spatial modeling capabilities of geographic information systems could extend the use of remote sensing beyond the research community into operational disease surveillance and control. An increasing number of health studies have used remotely sensed data for monitoring, surveillance, or risk mapping (Emch et al., 2008; Kelly-Hope et al., 2008; Pascual et al., 2000; Reyburn et al., 2011, Jutla et al., 2013). Heavy rainfall, river level, blooms of plankton, floods, coastal salinity, dissolved organic material, and increased sea surface temperatures have been 
identified as the main environmental risk factors that could increase the risk of cholera (Bompangue et al., 2009). Endemic cholera was linked with intrusion of vibrios from coastal regions to inland water bodies (Akanda et al., 2009). Hydro-climatological conditions such as increased rainfall, high temperature along with societal constrains such as inadequate water and sanitation services play a very effective role in making diarrhea more common by enhancing bacterial growth and survival and increases the likelihood of cholera outbreak (Mboera et al., 2012). High resolution environmental satellite data linked with diarrheal disease or other water borne disease are becoming increasingly available and accurate through calibration with real-time information on location (Ford et al., 2009). Availability of high resolution and accurate satellite data provides tremendous promise for prediction of water infectious diseases, weeks and even months in advance of an epidemic. In near future, increased computing power and spatial modeling capabilities of geographic information systems could extend the use of remote sensing beyond the research community into operational disease surveillance and control.

\subsection{Motivation and objective}

The motivation of my research is hinged upon the fact that bacteria (e.g. Vibrio cholerae, Vibrio vulnificus and Vibrio parahaemolyticus) are sensitive to water and several other climatic processes. Variability of hydroclimatic processes will dictate pathways of infectious diseases (e.g. cholera, vibrio infection) outbreak in human population. In addition, natural disasters often changes the variability of hydroclimatic processes or water use behaviors in human population and thus risking infectious diseases epidemics by multiple folds. As field observed data are expensive and unavailable in most places, hence satellite remote sensing based high resolution, near real time hydroclimatic estimations are extremely powerful for predictability of infectious diseases. Although the associations of several diarrheal diseases (e.g., cholera, shigellosis etc.) with climatic 
processes have been documented, the global human population remains at heightened risk of outbreak of diseases after natural disasters, such as hurricanes, earthquakes, floods or droughts. The uncertainties in timing and magnitude of natural disasters impact the hydroclimatic baseline, and/or access to safe drinking water and sanitation infrastructure (WASH). Also, data on disease prevalence and infectious pathogens is sparingly available in the region(s) where climatic variability and extreme natural events intersect with population vulnerability. Therefore, traditional time series modeling approach of calibration and validation of a model is inadequate and predictions of diarrheal infections remain a challenge. From this context, it is pivotal to understand the role of hydroclimatic processes in creating seasonality and inter-annual variability in environmental conditions favorable for exposure to pathogenic agents (e.g. bacteria) that lead to outbreaks of environmentally modulated water-related diseases. This study is motivated by the above noted concerns, and explores on how natural disasters expose the human population to natural pathways of bacteria by changing the variability of hydroclimatic processes or water use behaviors in human population, and thereby, risking infectious diseases epidemics by multiple folds? This research efforts to decode remotely sensed hydroclimatic big data and knowledge of large scale processes to help understand and predict infectious disease outbreaks.

The key goal of this research is to develop a remote sensing aided hydroclimatology driven cholera risk prediction on a global scale. Within this context, I focus on achieving the following three objectives

1. Development of a conceptual framework on assessing the impact of natural disasters with response to outbreak of water sensitive infectious diseases

2. Development of an algorithm capable of predicting cholera outbreak risk following a water sensitive natural disaster 
3. Exploration of the distribution, seasonality and proliferation risk of vibrios in aquatic environment

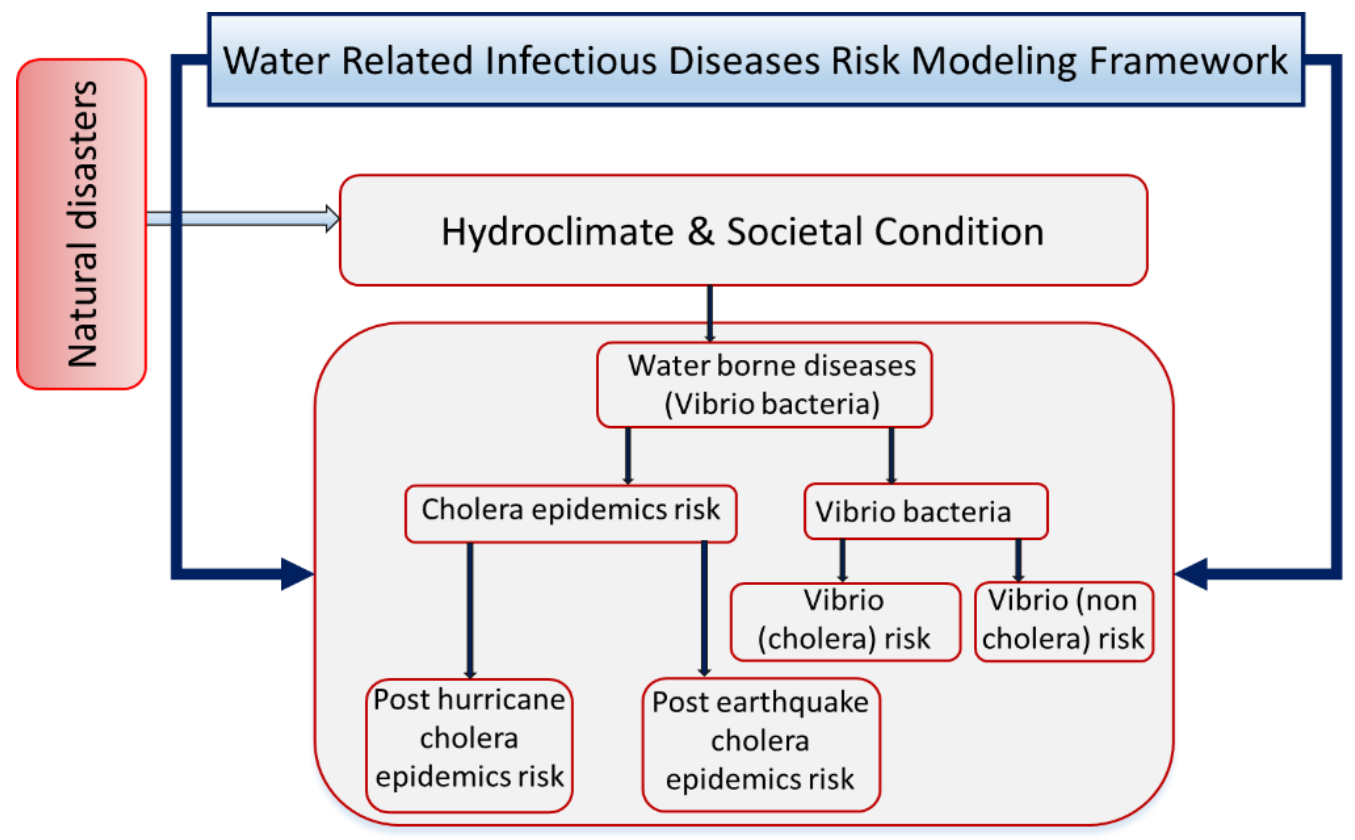

Figure 1.3: Remote sensing aided framework for predictability of water related infectious diseases in human population

\subsection{Outline of the dissertation}

The dissertation consists of six chapters including "Introduction" as chapter 1 and "Conclusion" as chapter 6. Chapter 2 to Chapter 5 are predominantly collections of published and in review papers as follows.

Chapter 1: Introduction

Chapter 2: A review paper on natural disaster and cholera epidemics, published in the "Journal of Environmental Health Reports" (doi: 10.1007/s40572-017-0132-5) 
Chapter 3: A published paper in "The American Journal of Tropical Medicine and Hygiene" that developed and applied a geospatial algorithm for real time cholera forecasting using NRT GRM precipitation following hurricane Matthew. (doi:10.4269/ajtmh.17-0048)

Chapter 4: A published paper in "Journal of Water Resources Planning and Management" that highlighted the role of resilient WASH infrastructure in preventing a cholera outbreak even when there were favorable hydroclimatic conditions for the survival of pathogenic cholera bacteria in natural water systems in the aftermath of the earthquake in Nepal in 2015. (doi.org/10.1061/(ASCE)WR.1943-5452.0000929)

Chapter 5: This paper is currently revised for "Journal of Environmental Engineering" that performs a detection and exploratory analysis of pathogenic vibrios across water, oyster and sediment in the Chesapeake Bay, Maryland

Chapter 6: Conclusion. A perspective article on real time choler monitoring in Yemen is currently in review in the "Journal of npj Clean Water" 


\title{
CHAPTER 2: NATURAL DISASTERS AND CHOLERA OUTBREAKS: CURRENT UNDERSTANDING AND FUTURE OUTLOOK
}

\begin{abstract}
Diarrheal diseases remain a serious global public health threat, especially for those populations lacking access to safe water and sanitation infrastructure. Although association of several diarrheal diseases, e.g., cholera, shigellosis etc, with climatic processes has been documented, the global human population remains at heightened risk of outbreak of diseases after natural disasters, such as earthquakes, floods or droughts. In this review, cholera was selected as a signature diarrheal disease and the role of natural disasters in triggering and transmitting cholera was analyzed. Key observations include identification of an inherent feedback loop that includes societal structure, prevailing climatic processes, and spatio-temporal seasonal variability of natural disasters. Data obtained from satellite based remote sensing are concluded to have application, although limited, in predicting risks of a cholera outbreak(s). This study argues that with the advent of new high spectral and spatial resolution data, earth observation systems should be seamlessly integrated in a decision support mechanism to be mobilized when a region suffers a natural disaster. A framework is proposed that can be used to assess the impact of natural disasters with response to outbreak of cholera, providing assessment of short -and long-term influence of climatic processes on disease outbreaks is human.
\end{abstract}




\subsection{Introduction}

The World Health Organization (WHO) defines natural disasters as "catastrophic events with atmospheric, geologic and hydrologic origins"(World Health Organization, 2006). These disasters include earthquakes, volcanic eruptions, storm surges, extreme temperatures, landslides, tsunamis, wildfires, floods, and droughts(EM DAT, 2016). An estimate derived from the International Disaster Database(EM DAT, 2016) suggests that about 270 million people are affected, annually, by natural disasters. Assessment of economic losses by continent, from 1960 to 2014, shows that Asia and the Americas suffer heavy monetary losses from natural disasters. Similarly, a disproportionate number within the human population is affected in Asia, followed by Africa.

Water-borne diarrheal diseases ,e.g., cholera, shigellosis and rotavirus infection, remain a leading cause of human mortality worldwide (World Meteorological Organization, 2012). Diarrheal infections are unlikely to be eradicated since many of the disease causing agents are either autochthonous to, or contaminants in, the aquatic environment (Constantin de Magny et al., 2008) and the infections, in turn, are linked to climatic processes. For example, cholera, a signature diarrheal disease and focus of this review, is transmitted via drinking water contaminated with Vibrio cholerae (Reidl and Klose, 2002). Cholera, an ancient disease, continues to be a major public health threat affecting 3 to 5 million people and causing 100,000 deaths annually(Zuckerman et al., 2007). Cholera has been classified to occur in three forms: epidemic, endemic and mixed-mode epidemics are defined as the sudden occurrence of cholera, generally observed in regions with limited connection to coastal waters. A persistent presence of human cholera cases with seasonal recurrent outbreaks, is termed endemic cholera. Mixed-mode is 
observed in regions that fall within the boundary of epidemic and endemic cholera (such as Dhaka, Bangladesh). Cholera is treatable, including oral rehydration therapy to replace water and electrolytes lost (Alexander et al., 2013). Unfortunately, mortality related to cholera in regions affected by natural disasters can be as high as $6 \%$ (Enserink, 2010a), compared to $0.1 \%$ in regions where a sanitation infrastructure exists.

Environmental factors, including precipitation, salinity, temperature, and nutrients are associated with persistence and growth of cholera bacteria in the aquatic environment(Alam et al., 2006a; Epstein, 1993; Singleton et al., 1982). Cholera bacteria comprise a component of the commensal flora of zooplankton, and form biofilms(Colwell, 1996a; Reidl and Klose, 2002). Copepods, often a dominant component of Zooplankton in the aquatic environment, feed on phytoplankton and high correlation has been reported between occurrence of copepods and phytoplankton blooms, with observed abundance in phytoplankton followed by Zooplankton blooms, and subsequent increase in cholera bacteria in nutrient rich water. Outbreaks of cholera over the last several decades in South Asia, Africa, and South America have occurred mostly along coastal areas (Colwell, 1996a; Constantin de Magny et al., 2008; Jutla et al., 2010). While the coastal regions remain the largest natural reservoirs of vibrios, epidemiological evidence suggests an increase in cholera incidence inland as well(Rebaudet et al., 2013). Analysis of the World Health Organization cholera reporting database (Figure 2.1) indicates that almost the entire African continent has reported cholera over the past 20 years, with inland regions experiencing outbreaks of the disease. For example, Zimbabwe experienced a massive cholera outbreak in 2008-2009, with more than 100000 victims("WHO | Cholera in Zimbabwe - update," n.d.) seeking treatment at medical facilities. Similarly, non-coastal regions of Mozambique, Rwanda, Cameroon and South Sudan reported significant number of cholera cases in recent decades(Jutla et al., 2015). 


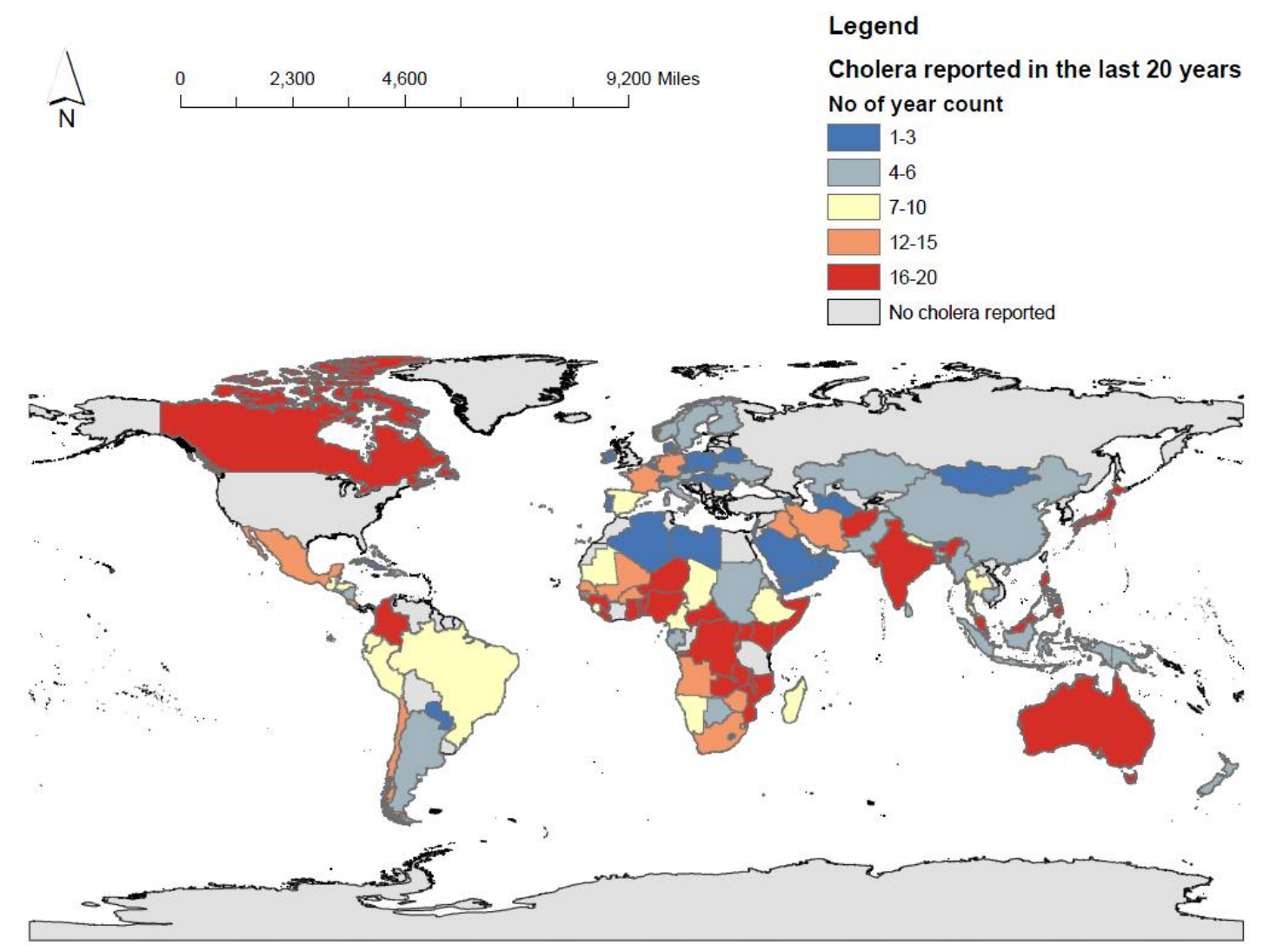

Figure 2.1: Cholera map showing number of years of reported cholera patients in WHO database.

Three empirical observations motivate the exploration of possible connections of natural disasters with the ecology of vibrios in the aquatic environment: (i) laboratory and field studies suggest significant correlation between plankton abundance and pathogenic cholera bacteria (Alam et al., 2006b; Huq et al., 2005; Singleton et al., 1982); (ii) changing climate has now been linked to increased incidence of natural disasters, resulting in stress on water availability in regions where population vulnerability interacts with hydroclimatic extremes(Leaning and Guha-Sapir, 2013; McMichael, 2009) and (iii) changes in intensity and seasonality of hydroclimatological processes can be expected to affect ecological niches of vibrios. Association of a natural disaster with diarrheal disease, notably cholera, is the subject of debate since not all disasters result in 
outbreaks of cholera(Brown and Murray, 2013). With a somewhat limited efficacy of vaccines and the overall health threat posed by cholera, it is important to understand the dynamics of the disease, both its reservoir in the environment and the detrimental effects of cholera epidemics on society (Emch et al., 2008). Therefore, the goal of this review is to document known relationships between natural disasters and cholera. Within this context, a schematic futuristic framework is provided for estimating the likelihood of an outbreak of cholera in a region suffering a natural disaster.

\subsection{Natural disasters and cholera epidemics}

Natural disasters affect the ecology of pathogens, mainly by increasing the risk of exposure to pathogens (Bartlett, 2008). Congested human communities and refugee camps have greater risk of cholera, primarily from the exposure of the human population to vibrios naturally occurring in the aquatic ecosystem and poor sanitation infrastructure, and a greater probability of unsafe drinking water (World Health Organization, 2006).

The current pathway for potential outbreaks of diarrheal diseases in human populations following a natural disaster is often misinterpreted, leading to chaos in the population (PanAmerican Health Organization, 2000). For example, following a deadly earthquake in Nepal in 2015, there were widespread reports of an impending cholera outbreak in the region. However, except for few cases, the region remained free of cholera epidemics. Yet, a massive cholera outbreak occurred in Haiti following the devastating earthquake of 2010 (Enserink, 2010b). In terms of economics, Haiti has higher per capita income (\$819) than Nepal (\$694), yet Haiti suffered severely from the earthquake compared to Nepal, essentially a paradox with respect to environmental understanding of the role of natural disasters related to outbreaks of cholera. The predominant view of spread of diarrheal diseases after a natural disaster is that disrupted or destroyed infrastructure, e.g., water purification and sanitation infrastructure, will result in disease 
outbreaks (Bartlett, 2008). However, not all disasters are associated with cholera epidemics. In fact, the conclusion of several studies (Noji, 1997) is that disease outbreaks are less frequently reported in disaster affected regions and are more likely to occur in conflict zones, where the majority of infections result are caused by various forms of communicable disease agents. Yet, relatively few natural disasters shock an otherwise normal societal system sufficiently catastrophic to result in a surge in the diarrheal disease burden (Watson et al., 2007).

The threat of disease immediately after a natural disaster in a region is, in fact, low, but if the societal structure is not sufficiently resilient to bounce back from the shock of the disaster, a massive diarrheal disease outbreak may occur. For example, the Pan American Health Organization has categorized the effects of disasters on human health when disruption of societal services also occurs (Table 1). What was concluded is that the risk of damage to water sources following an earthquake is very high. However, if the infrastructure is quickly rebuilt, then the chances of a diarrheal disease outbreak diminishes significantly. Human populations in those areas where access to electricity, clean water, and sanitary facilities is limited are especially susceptible to acute diarrheal disease that can spread rapidly, with very high mortality rates across all age groups. After a natural disaster, people are often forced to seek temporary shelter where conditions are crowded and sanitation and waste management is inadequate. Compromised sources of water result and when coupled with low levels of immunity in children and the elderly, outbreaks of cholera can be catastrophic (Cash et al., 2013). All of the above arguments are valid under the assumption that the infectious dose of a pathogen, such as $V$. cholerae occurs in the drinking water supply system. 
Table 2.1: Common effects of natural disasters on societal services (Source: PAHO)

\begin{tabular}{|c|c|c|c|c|c|c|}
\hline \multirow{7}{*}{$\begin{array}{l}\text { Water supply and } \\
\text { wastewater disposal }\end{array}$} & & 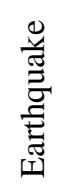 & 苞 & $\frac{\overrightarrow{0}}{\overline{0}}$ & $\begin{array}{c}\vec{\Xi} \\
\stackrel{\Xi}{\Xi} \\
\stackrel{\Xi}{\bullet}\end{array}$ & 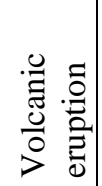 \\
\hline & Damage to civil engineering structures & 1 & 1 & 1 & 3 & 1 \\
\hline & Broken mains & 1 & 2 & 2 & 1 & 1 \\
\hline & Damage to water sources & 1 & 2 & 2 & 3 & 1 \\
\hline & Power outages & 1 & 1 & 2 & 2 & 1 \\
\hline & Contamination (biological) & 2 & 1 & 1 & 1 & 1 \\
\hline & Transportation failures & 1 & 1 & 1 & 2 & 1 \\
\hline \multirow{5}{*}{ Solid waste handling } & Damage to civil engineering structures & 1 & 2 & 2 & 3 & 1 \\
\hline & Transportation failures & 1 & 1 & 1 & 2 & 1 \\
\hline & Equipment shortages & 1 & 1 & 1 & 2 & 1 \\
\hline & Personnel shortages & 1 & 1 & 1 & 3 & 1 \\
\hline & Water, soil, and air pollution & 1 & 1 & 1 & 2 & 1 \\
\hline \multirow{4}{*}{ Home sanitation } & Destruction or damage to structures & 1 & 1 & 1 & 1 & 1 \\
\hline & Contamination of water & 2 & 2 & 1 & 2 & 1 \\
\hline & Disruption of water supply system & 1 & 1 & 1 & 2 & 1 \\
\hline & Overcrowding & 3 & 3 & 3 & 3 & 2 \\
\hline
\end{tabular}

1 - Severe possible effect

2- Less severe possible effect

3- Least or no possible effect

Outbreaks of cholera (epidemic, endemic, or mixed mode) can be limited to two components: the triggering and the transmission of the disease in a human population (Joh et al., 2009; Koelle et al., 2005). Consumption of water containing an infective dose of the cholera bacteria can trigger cholera outbreak in a population. However, growth of the pathogen is linked to climatic factors with strong seasonality (Akanda, 2011; Colwell, 1996b; Faruque et al., 2005; Finger et al., 2014) that further affects the ecological niche of the vibrio. Therefore, following a natural disaster, an outbreak may not occur unless environmental conditions are favorable to rapid growth and massive proliferation in the aquatic water system. Once the trigger of disease is initiated, transmission of cholera is accelerated by fecal-oral transmission, and ultimately, a disease 
outbreak. For example, hydroclimatological conditions namely, warm temperatures preceding a heavy rainfall, and subsequent inadequate safe water supply and poor sanitation will increase the likelihood of a cholera outbreak (Faruque et al., 2005; Jutla et al., 2015; Rinaldo et al., 2012). A natural disaster can act as a catalyst in the interaction of contaminated water and the human population. The lack of safe water coupled with the breakdown in sanitation services in a country already challenged by an inadequate safe water supply and poor sanitation infrastructure resulted in the massive cholera epidemic in Haiti, with 658,563 human cases and 8,111 reported deaths during the time period of 2010 to 2013 (Gelting et al., 2013).

A massive cholera epidemic occurred in West Bengal (with more than 16,000 human cases) in 1998 it was attributed to the flooding that occurred prior to the outbreak (Sur et al., 2000). Floods in Mozambique occurred in 2000 and was followed by an increase in the incidence of diarrhea (Kondo et al., 2002). More than 350,000 cases of diarrhea were reported after the July 2004, floods in Bangladesh (Watson et al., 2007). In the United States, after hurricane Allison, approximately 1,000 cases of diarrhea and vomiting among adults and children were reported in Mississippi and Texas (Waring et al., 2002). Clearly, with an increasing population, safe drinking water sources and effective sanitation infrastructure are important and effective means of preventing outbreaks of cholera and diarrheal diseases in general (Cairncross et al., 2010).

Although the cholera epidemic that occurred in Haiti can be linked to the destruction of water and sanitation infrastructure after the earthquake and the continued unhygienic living conditions in refugee camps, a similar disaster that occurred in Pakistan in 2005 reportedly did not lead to a massive cholera outbreak. In contrast, following flooding in Pakistan during 2010, more than 600,000 people sought treatment for diarrheal disease. A key difference between the 2005 and 2010 Pakistan disasters was that environmental conditions favorable to bacterial growth and 
proliferation were present in 2010 and not in 2005. The 2005 Pakistan flooding occurred just before the winter and in a mountainous region where it was extremely cold and not conducive to growth of the cholera bacteria in the water, hence spread of V. cholera was raised. Conversely, in August, 2010, floods occurred in the flatlands of Pakistan where conditions were optimal for growth and dissemination of cholera bacteria. However, a major cholera epidemic did not occur after the severe hurricane that battered the Gulf Coast of the United States in 2005, raising a question that, despite Hurricane Katrina, a cholera did not arise in Louisiana, a state severely impacted by the event. Over the past five years, a total of 44 cases of cholera have been reported in the United States, but none of these cases were followed by an outbreak of cholera. It is concluded that the robust and resilient water and sanitation infrastructure coupled with effective prevention methods benefited spread of the disease. The 2015 Nepal earthquake affected a significant portion of the human population, with extensive damage to the sanitation infrastructure and possible diarrheal disease (Nelson et al., 2015).

The question remains why are some regions of the world subject to cholera epidemics after a natural disaster and other such regions not. A possible explanation, apart from favorable environmental conditions for growth of the cholera bacteria, is the time scale related to resilience of some human communities to resist an outbreak. There are two time-based pathways (Figure 2.2), short (black arrows) and long (red arrows) term, by which environmental conditions favorable for diarrheal disease following a disaster can be assessed. The short term pathway is initiated immediately after a disaster and is a function of prevailing weather patterns associated with growth of the pathogen in the aquatic ecosystem. $V$. cholerae interacts with humans through a link function, termed societal response, which includes safe water and sanitation access, hygiene practices, and water security in a given population. Should the societal response function be 
compromised, a trigger of cholera may be imminent. However, the disease may not spread in a human population, unless a human to environment (through pathogen shedding) link is established. Once transmission of the disease begins, a feedback loop between pathogen and humans is activated, resulting in a massive outbreak of disease. The short term pathway has been explored in several recent studies (Codeco et al., 2008; Jutla et al., 2015; Mari et al., 2012; Mukandavire et al., 2013; Pascual et al., 2002), where it was shown that an outbreak of cholera is not possible unless there are sufficient infected human cases in the population. Within the long-term pathway trigger of disease (Figure 2.2), there is the function of how rapidly the human population can be resettled after the disaster and before human interactions occur, notably with unsafe water containing pathogenic vibrios and an association with seasonal climatic processes.

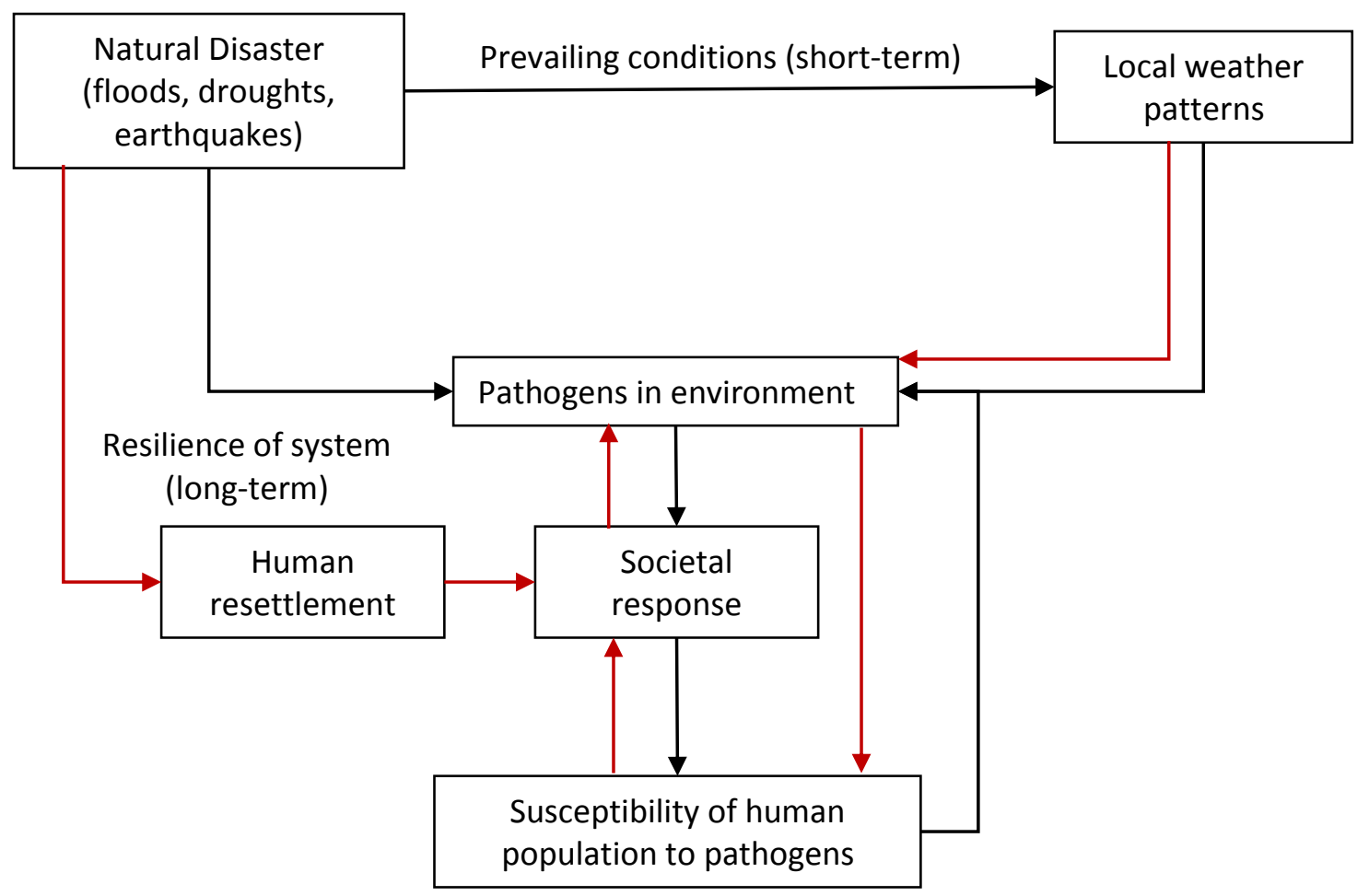

Figure 2.2: Framework to understand environmental pathways for an outbreak of cholera after natural disaster 
A seminal study published by the WHO (Pan-American Health Organization, 2000) reported that a short burst of relief effort in a disaster hit population will have limited effect, since the post-effects of disaster last for a long time. The study also concluded that the local population almost always covered itself with lifesaving needs on a short term basis. Therefore, environmental conditions under which the human population can interact with vibrios over time must be closely monitored. A relevant example is the October, 2010, cholera outbreak in Haiti that occurred after the earthquake hit the region in January of 2010. A curious observation is that there was a gap of almost ten months between the earthquake and outbreak of cholera. The month of January is cold in Haiti, limiting growth of vibrios in the aquatic system. However, as the weather warmed in September ( with an anomalous increase in temperature), growth of pathogenic vibrios was enhanced (A. Jutla et al., 2013a). Societal response did not improve during the ten preceding months following the earthquake, with the consequence of increased interaction of human population with water containing vibrios. Based on previous studies, providing safe water and sanitation to the human population following a disaster is critical (Fuller et al., 2014; Wutich and Ragsdale, 2008). If the water infrastructure is resilient or repaired effectively after a natural disaster, the risk of cholera will be low.

\subsection{Future outlook: Predictability of cholera}

A very important challenge is to be able to determine the risk of an outbreak following a natural disaster. Since the effectiveness and availability of cholera vaccines are not absolute, accurate prediction of cholera epidemics will permit effective application of public health measures to restrict both initiative and spread of the disease. Conditions favorable to growth of the cholera vibrio based on climatic processes, offers the possibility of monitoring short- and longterm pathways triggering cholera. Several statistical models, e.g., correlation and simple 
regression (A. S. Jutla et al., 2013; Masuda and Holme, 2013), logistic regression (Bingham et al., 2004; Nguyen et al., 2014), multivariate regression (Colombara et al., 2013; Harris et al., 2009), Poisson regression (Huq et al., 2005), artificial neural network (Penna, 2004), and Bayesian regression (Osei et al., 2012) offer promising results for prediction and simulation of cholera outbreaks. Spatial scale prediction of the probability of disease risk can be achieved using the Geographical Information Systems (GIS) (Chiang et al., 2013).

Given the many available tools, the continuing challenge is obtaining data on the hydroclimatology of a region affected by natural disaster. Earth observing satellites offer a novel solution for monitoring, at different spatial and temporal scales, the ecological abundance of pathogenic vibrios by associating abundance with climatic processes. The task of linking satellite based earth observations with the ecology of the cholera bacteria is an emerging area of research proving to be immensely useful when a serious natural disaster hits a region. Previous studies (Bompangue et al., 2008; Emch et al., 2008; Griffith et al., 2006; A. Jutla et al., 2013b; Pascual et al., 2002; Reyburn et al., 2011) reported that rainfall, water level, plankton abundance, inundation, coastal salinity, dissolved organic material, and sea surface temperatures, are potential factors influencing the ecology of the cholera bacteria, hence cholera. Most hydroclimatic and ecological processes influencing the growth of $V$. cholerae can be estimated using satellite earth observation sensors with reliable accuracy. Table 2.2 provides a summary of the key studies that have quantitatively used satellite remote sensing data for quantitative analysis to understand the trigger of diarrheal disease (including cholera). Most of the studies were conducted during the past decade, perhaps an indication that as more data become available from satellite sensors, predictive linkage of diarrheal disease with hydroclimatic processes will be strengthened. 
Table 2.2: Diarrheal diseases modeling around the globe using remotely sensed data

\begin{tabular}{|c|c|c|c|c|}
\hline Disease & Variable & Satellite & Author & Study area \\
\hline Cholera & $\begin{array}{l}\text { Ocean chlorophyll } \\
\text { concentration, Sea surface } \\
\text { height, Sea surface } \\
\text { temperature }\end{array}$ & $\begin{array}{l}\text { SeaWiFS, } \\
\text { TOPEX/Poseid } \\
\text { on ,AVHRR }\end{array}$ & Xu M. et al 2016 & China \\
\hline Cholera & Precipitation & TRMM & Jutla A.S. et al 2015 & Zimbabwe \\
\hline Cholera & Precipitation & TRMM & Kirpich et al. 2015 & Haiti \\
\hline Diarrheal & $\begin{array}{l}\text { Total water storage (TWS) } \\
\text { anomalies }\end{array}$ & GRACE & Jutla A.S. et al 2015 & $\begin{array}{l}\text { Ganges } \\
\text { Brahmaput } \\
\text { ra Meghna } \\
\text { basin }\end{array}$ \\
\hline Cholera & Ocean color L1A data & MODIS aqua & Knox A. et al 2014 & East Africa \\
\hline Diarrheal & NDVI & $\begin{array}{l}\text { AVHRR, } \\
\text { MODIS }\end{array}$ & $\begin{array}{l}\text { Brown M. E. et al } \\
2014\end{array}$ & Nepal \\
\hline Cholera & Precipitation & TRMM & Eisenberg et al 2013 & Haiti \\
\hline Cholera & $\begin{array}{l}\text { Chlorophyll Concentration, } \\
\text { CDOM }\end{array}$ & SeaWiFS & Jutla A.S. et al 2013 & $\begin{array}{l}\text { Bengal } \\
\text { delta }\end{array}$ \\
\hline Diarrheal & Images & Radarsat-2 & Yomwan P. et al 2012 & $\begin{array}{l}\text { South east } \\
\text { Africa }\end{array}$ \\
\hline Rotavirus & NDVI & MODIS & Jagai J.S. et 12012 & South Asia \\
\hline $\begin{array}{l}\text { Cryptospori } \\
\text { diosis }\end{array}$ & NDVI & MODIS & Jagai J.S. et 12008 & $\begin{array}{l}\text { Sub- } \\
\text { Saharan } \\
\text { Africa }\end{array}$ \\
\hline Cholera & $\begin{array}{l}\text { Sea surface temperature } \\
\text { Sea surface height, } \\
\text { Chlorophyll Concentration, }\end{array}$ & $\begin{array}{l}\text { AVHRR, } \\
\text { TOPEX/Poseid } \\
\text { on, } \\
\text { SeaWiFS }\end{array}$ & Emch M. et al 2008 & $\begin{array}{l}\text { Bengal } \\
\text { delta }\end{array}$ \\
\hline Cholera & Chlorophyll Concentration & SeaWiFS & De Magny et al 2008 & $\begin{array}{l}\text { Bengal } \\
\text { delta }\end{array}$ \\
\hline Cholera & Images & SPOT & Ali M. et al 2002 & $\begin{array}{l}\text { Bengal } \\
\text { delta }\end{array}$ \\
\hline Cholera & $\begin{array}{l}\text { Sea surface temperature, } \\
\text { Sea surface height }\end{array}$ & $\begin{array}{l}\text { AVHRR, } \\
\text { TOPEX/Poseid } \\
\text { on }\end{array}$ & Lobitz et al 2000 & $\begin{array}{l}\text { Bengal } \\
\text { delta }\end{array}$ \\
\hline
\end{tabular}

\subsection{Discussion}

In a seminal study, it was argued that quantitative understanding of environmental changes impacting human health is significantly understudied (McMichael, 2009), evident from the fact that very few reports link availability of safe water with infectious diseases. The focus of this 
review article, highlighting the role of natural disasters on triggering and transmission of diarrheal diseases, emphasized cholera a global problem. Two of the conclusions of this study are: (i) linkages between natural disasters and cholera are highly non-linear and not yet well understood; and (ii) prediction of cholera employing satellite sensors can be useful in mitigating the effects of such disasters on the human population. As complementary evidence, a search of scientific, peerreviewed journal articles on cholera, employing relevant keywords (sustainability, disaster, natural disaster, floods, drought, earthquake, hazard) was done using SCOPUS (search engine). Results are presented Table 3, showing that very few studies have been done. "Cholera and Disaster" as a keyword, yielded the highest search return (96 articles). However, the majority of these articles were published only very recently (within the last decade). In addition, they did not elaborate a physical hypothesis concerning the impact of natural disasters on cholera outbreaks, indicating that our understanding of the impact of natural disasters on cholera is emerging. With new satellite data observations becoming available, this research will provide valuable insight on climate and human disease.

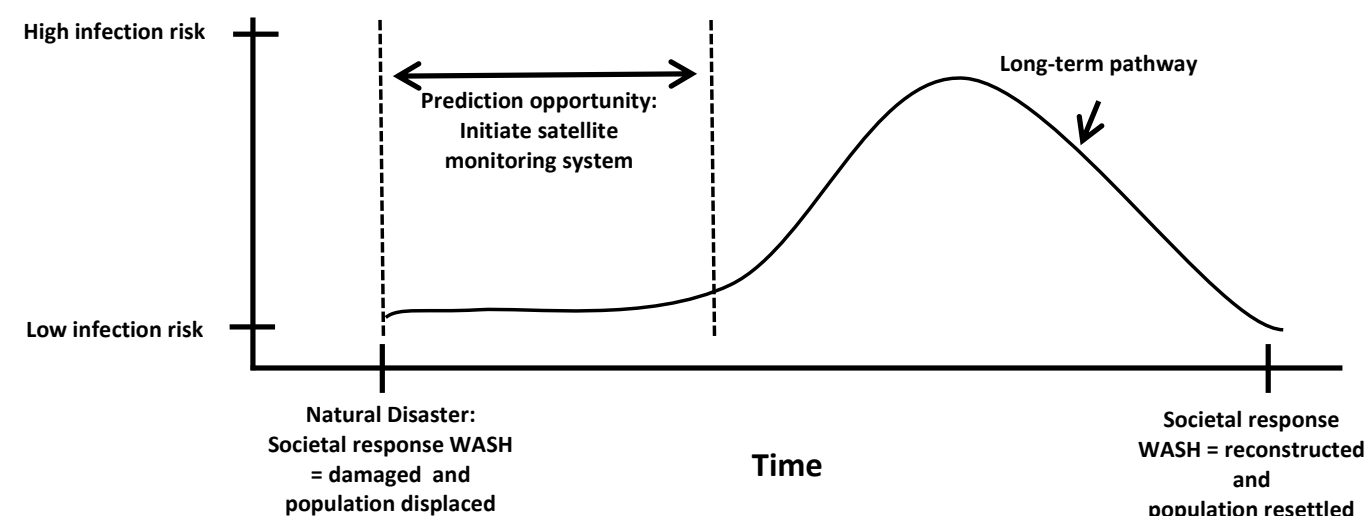

Figure 2.3: Timeline of natural disaster and cholera 
Table 2.3: Results of web search using SCOPUS on cholera and several related keywords in abstracts

\begin{tabular}{|l|c|}
\hline Cholera and & Articles \\
\hline Sustainability & 19 \\
\hline Disaster & 96 \\
\hline Natural disaster & 34 \\
\hline Floods & 43 \\
\hline Drought & 31 \\
\hline Earthquake & 50 \\
\hline Hazard & 62 \\
\hline
\end{tabular}

Results in all published literature suggest that the risk of cholera (or diarrheal disease) occurring immediately after a natural disaster is low in general. However, the risk increases if the appropriate societal response function (availability of WASH: water, sanitation and hygiene) is not improved over time. Without an effective vaccine, and lack of safe water and sanitation predictability of risk of cholera assumes greater importance. In Figure 2.3, summary timeline for occurrence of cholera occurrence after a natural disaster is shown. The long-term pathway of cholera is indicated and showing opportunity time with accurate prediction of disease, if an appropriate satellite monitoring system (data collection and analysis) is engaged immediately after the disaster. The assumption is that models or relationships of cholera with hydroclimatic processes exist. In any case, it is clear that the primary effort should be on providing safe water and sanitation infrastructure to the human population on a sustained basis to prevent (or ameliorate) an outbreak of cholera. In summary, based on past and present experience, both epidemiological surveys and prediction models for waterborne disease effectively enhance public health monitoring and provide the opportunity for enhanced response to infectious disease outbreaks, most effectively when the natural disasters disrupt infrastructure of the social system. 


\title{
CHAPTER 03: ASSESSMENT OF RISK OF CHOLERA IN HAITI FOLLOWING HURRICANE MATTHEW
}

\begin{abstract}
Damage to the inferior and fragile water and sanitation infrastructure of Haiti after Hurricane Matthew has created an urgent public health emergency in terms of likelihood of cholera occurring in the human population of Haiti. Using satellite derived data on precipitation, air temperature, and hurricane path, and, with information on water and sanitation (WASH) infrastructure, this research $\mathrm{h}$ tracked changing environmental conditions conducive for growth of pathogenic vibrios. Based on these data the likelihood of cholera cases occurring past hurricane were predicted and validated. The risk of cholera occurring in the South western part of Haiti has remained relatively high since November 2016 to the present. Findings of this study provide a contemporary process for monitoring ground conditions that can guide public health intervention to control cholera such as provision of vaccines, safe water and sanitation (WASH). Assuming current social and behavioral patterns will remain constant, it is recommended that WASH infrastructure should be improved and considered a priority prior to the especially 2017 rainy season.
\end{abstract}




\subsection{Introduction}

Prediction of an outbreak of diarrheal disease, specifically cholera, following a natural disaster remains a challenge, especially in regions lacking safe water, sanitation and hygiene (WASH) infrastructure. The underlying mechanism of a cholera outbreak is linked to disruption in the WASH infrastructure that results in the human population lacking safe water free of pathogens. Previous research (Alam et al., 2006; Ali M et al 2002;Cash B et al., 2014; Faruque S et al., 2005; Hashizume M et al., 2009; Huq A et al., 2005; Jutla A et al., 2015; Rinaldo A et al., 2014; Singleton F et al., 1982) showed that the presence and abundance of vibrio cholera, the causative agent of cholera, are related to modalities of the environment and regional weather variability as well as the climate systems (Pascual M 2000; Jutla A et al., 2013). Major cholera outbreaks occur in two dominant forms: (a) epidemic, characterized by a sudden and sporadic occurrence of a large number of cholera cases and (b) endemic, in which human cholera cases occur on annual scales with distinct and characteristic seasonality. Our dominant hypothesis for epidemic cholera is that an elevated temperature followed by heavy precipitation, in concatenation with an insufficient and damaged WASH infrastructure, places the human population at high risk of interaction with cholera bacteria and subsequent by the outbreak of the disease (Jutla A et al., 2015; Akanda A et al., 2013).

Natural disasters characteristically leave a trail of destruction, the result of which may be a human population deprived of access to WASH (Barlett J, 2008). For example, under normal circumstances, the likelihood of a cholera outbreak is low, since the human population adapts to it's specific behavioral pattern of water use. However, following a natural disaster, human behavior will change, if the availability, use pattern, and storage capacity of drinking water are altered as a result of the WASH infrastructure having been severely damaged and/or rendered 
unusable (Watson J et al., 2007). Case in point, Hurricane Matthew lashed Haiti with heavy rainfall for three days, from October 2 to 4, 2016. The question that arises is whether the particular region hit by hurricane will be at risk for a cholera epidemic. In addition, can appropriate intervention and mitigation be identified and communicated to relevant policy-makers. Forecasting a cholera outbreak is challenging because of the lack of data on pathogen abundance in local water systems, weather and climate patterns and existing WASH infrastructure. Vibrios, including V. cholerae are autochthonous to the natural aquatic ecosystem, hence eradication is not feasible ${ }^{16}$. Therefore, the objective of this research was to employ our current hypothesis of the epidemic form of cholera to forecast probabilistic risk of the disease occurrence in Haiti after Hurricane Matthew. The utility of high spatial and temporal resolution satellite products to identify regions requiring attention of policy-makers to institute cholera mitigation strategies was also explored.

\subsection{Materials and Methods}

\subsubsection{Data}

Monthly air temperature data at spatial resolution of $2.5^{0} \times 2.5^{0}$ space were obtained from the National Center for Environmental Prediction and daily precipitation data from the Global Precipitation Mission (GPM), accumulated to create a monthly dataset at $0.1^{0} \times 0.1^{0}$ resolution. Long term average precipitation was obtained using monthly precipitation data (3B43 product) from the Tropical Rainfall Measuring Mission (TRMM) at spatial resolution of $0.25^{0} \mathrm{x}$ $0.25^{0}$.Thereafter, the TRMM dataset was resampled to $0.1^{0} \mathrm{x} 0.1^{0}$ to align with the GPM dataset. Data on track points and wind swath of Hurricane Matthew were collected from the National Hurricane Center (NHC). Administrative boundaries, roads, and river data for Haiti were obtained from the Haiti- Grand Valley State University Program of Safe and Sustainable Water for Haiti (http://www.gvsu.edu/haitiwater/links-to-gis-data-for-haiti- 9.htm). Population density data 
(GPWv4) were acquired from the NASA Socioeconomic Data and Applications Center (SEDAC).Analysis was done using the R-programming language platform (v3.2.3) and ArcMap (v10.2.1)

\subsubsection{Spatial hydroclimatic analysis}

A theoretical pathway (Figure 3.1) was used to evaluate hydroclimatic control of the risk of cholera in Haiti. This pathway argues that the probability of epidemic cholera in a human population increases if the region experiences a two month above average air temperature, followed by one month of above average precipitation. These two conditions must be accompanied by damage to WASH infrastructure, further facilitating interaction of cholera bacteria with humans and thereby leading to an outbreak. We estimated monthly anomalies (defined as departure from an average condition) for both air temperature and satellite precipitation data, using the equation

1.

Monthly anomaly $=$ Monthly value Precipitation $/$ Temperature $_{\text {- Long term average value }}$ Precipitation/Temperature

(Eq 1)

For example, to calculate air temperature anomalies for a given month (e.g., September), an average for monthly data from 1981 to 2016 was determined and subtracted from the value for the corresponding month of year 2016. The resulting positive value (anomaly) implies that the month is warmer, based on the historical mean condition and vice versa. For estimation of a precipitation anomaly for a given month, an average monthly data value was estimated using TRMM data and subtracting it from the corresponding monthly GPM value for year 2016. As was determined for air temperature, a positive precipitation anomaly implied a wetter month, compared to the historical mean condition and vice versa. These anomalies were calculated for both monthly total precipitation and monthly mean air temperature, covering all pixels over Haiti. 


\subsubsection{Composite weighted raster overlay for cholera risk mapping}

We used a weighted raster overlay (WRO) algorithm to produce a risk map for cholera, risk being defined as the probability of occurrence of cholera in a region. The WRO algorithm is a geospatial method that applies a common measurement scale of values to generate an integrated composite from several dissimilar raster layers. Figure 3.2 shows simplified steps to apply this algorithm to develop cholera risk maps. The algorithm begins by selecting appropriate hydroclimatic and societal variables associated with the triggering of epidemic cholera. The dataset includes precipitation and air temperature anomalies, human population parameters, and hurricane wind-swath and track. Each raster layer was classified as a risk cluster and assigned an integer value based on a predefined evaluation scale (Table 3.1).

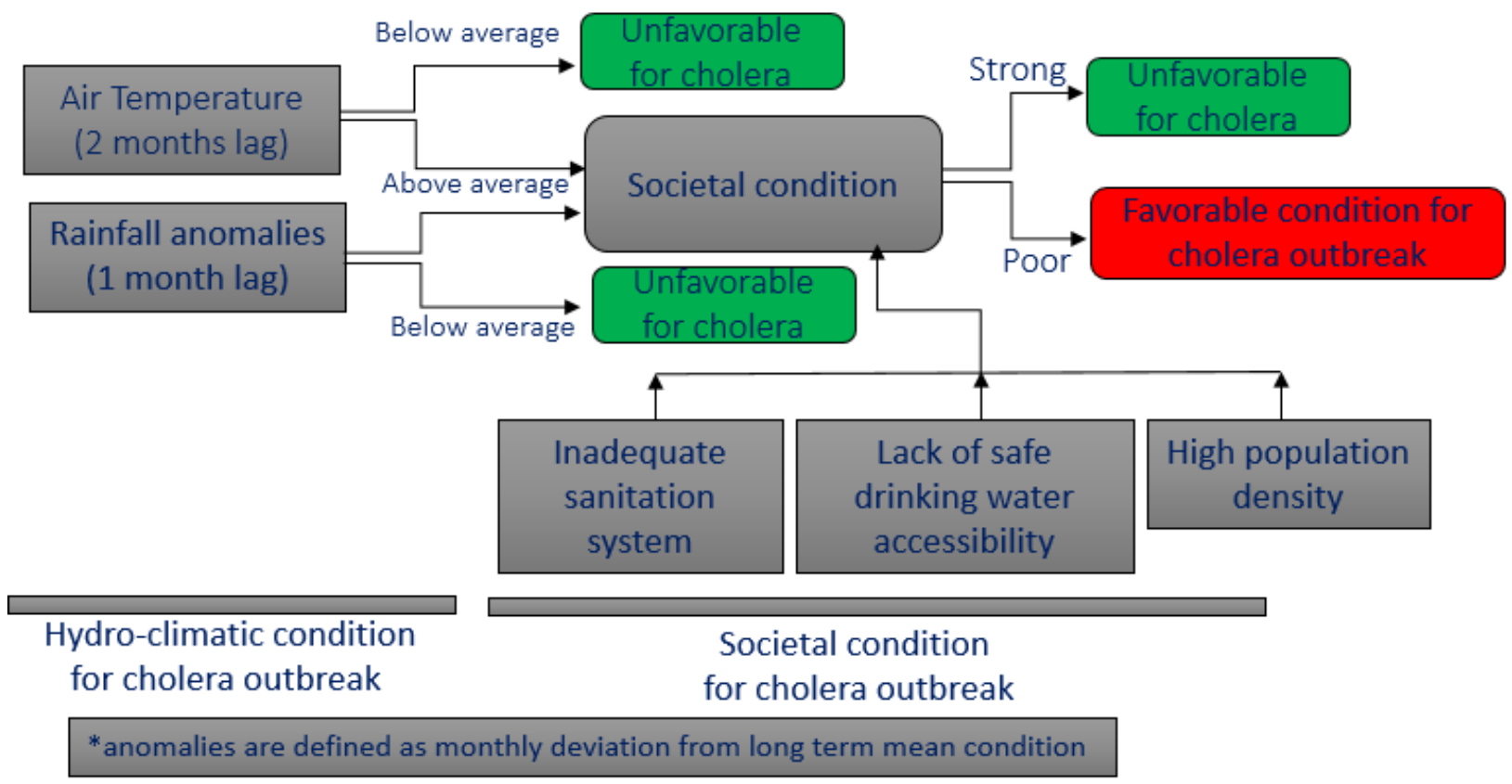

Figure 3.1: Epidemic cholera risk hypothesis 


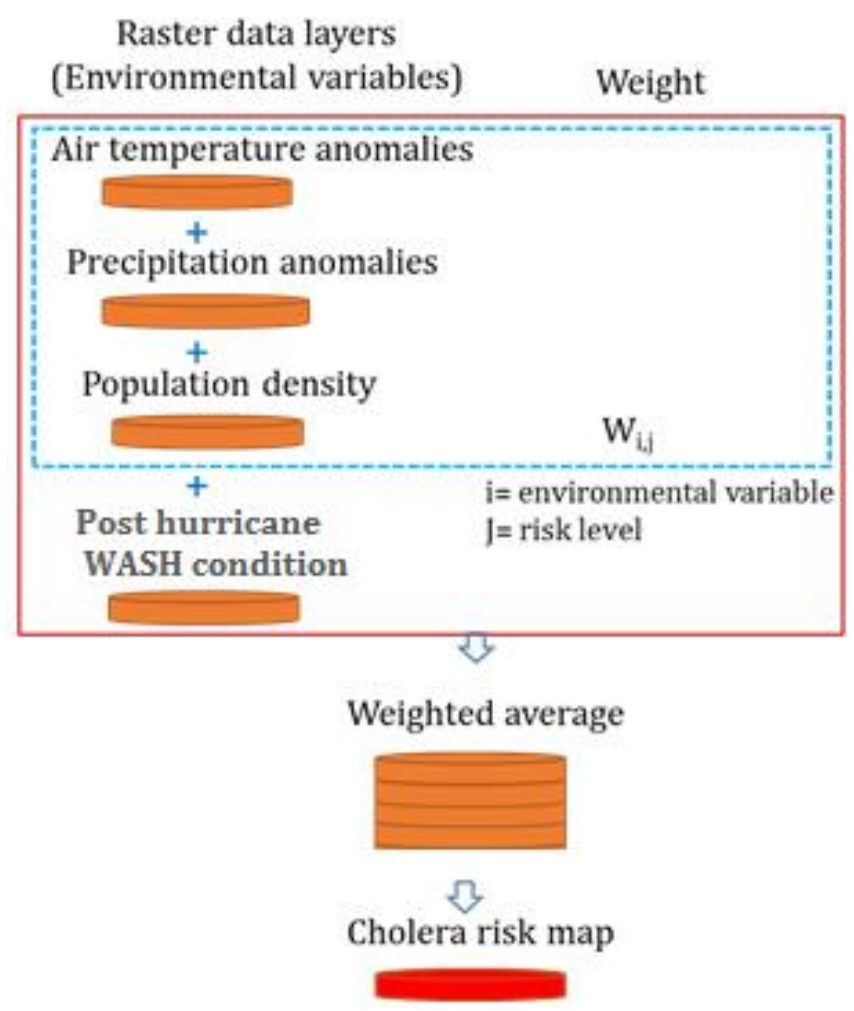

Figure 3.2: Composite weighted raster overlay algorithm.

For each variable, the maximum and minimum value of the evaluation scale represents two extreme risk conditions, e.g. very high or very low risk. The intermediate values of the evaluation scale represent in-between risk conditions for the variable of interest. That is, the newly generated values represent reclassification of the original raster values. Each raster layer was classified according to an evaluation scale of 0 (very low risk) to 4(very high risk). For precipitation, an anomalous condition greater than one standard deviation (66\% confidence interval based on analysis of historical data) from the mean (greater than $5 \mathrm{~mm} /$ day) was considered high risk for cholera and assigned a risk level value of 3 . An extreme of anomalous condition (greater than 15mm/day: six standard deviations representing $99 \%$ confidence interval) was categorized as a very high risk of cholera occurring and was assigned the risk level value of 4 . Similarly, for air temperature, an anomalous value greater than one standard deviation from the mean (greater than 
$1^{0} \mathrm{C}$ ) was to be a moderately high risk of cholera cases occurring and was assigned a risk level value of 2 . Extremely anomalous temperature values greater than $2^{0} \mathrm{C}$ were considered very high risk of a cholera outbreak occurring and was assigned a risk level value of 4. For wind swath, first fifty miles of the radius from the nearest path of a hurricane was considered nearest in proximity i.e 'closest'. Therefore it was considered to provide the highest risk of damage to WASH infrastructure and was assigned the risk level value of 4. Similarly, inland regions located within 50 to 100 miles, and 100 to 150 miles and 150 to 200 miles radius were assigned the risk levels of 3,2 and 1 respectively.

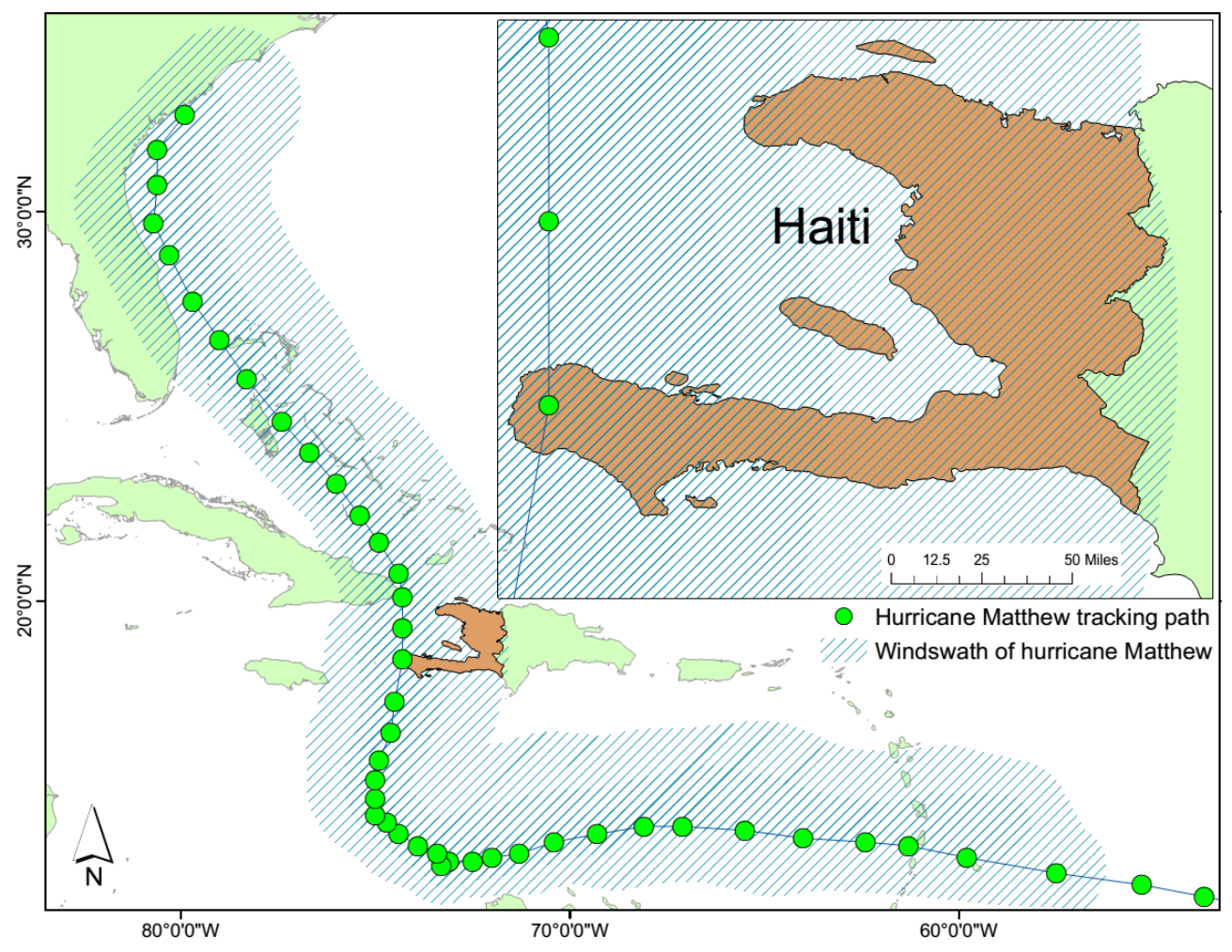

Figure 3.3: Hurricane Matthew tracking over Haiti.

Each of the reclassified variables was given equal weight and a multiplier to emphasize the relative importance of the process with respect to triggering a cholera outbreak. All weighted and reclassified raster layers were added to create a risk composite, representing the influence of each 
of the variables. To estimate risk for a given region, the composite was spread across the lowest possible administrative area polygon, e.g. level 3. The mean of the pixels within a polygon was assigned a risk value. The risk raster layer was rescaled between two values of the composite (e.g., above which the region was at very high and very low risk). The maps were then rescaled between 0 and1, an important step for future comparison of all the regional risk maps to the same scale.

Table 3.1: Risk classification for each layer

\begin{tabular}{lcccc}
\hline Risk level & $\begin{array}{l}\text { Precipitation } \\
\text { anomalies } \\
(\mathbf{m m} / \mathbf{d a y})\end{array}$ & $\begin{array}{l}\text { Temperature } \\
\text { anomalies (degree } \\
\mathbf{C})\end{array}$ & $\begin{array}{l}\text { Population } \\
\text { densities (count } \\
\left.\text { per } \mathbf{k m}^{2}\right)\end{array}$ & $\begin{array}{l}\text { Wind swath of } \\
\text { hurricane }\end{array}$ \\
\hline Very High & $4(>15)$ & $4(>2)$ & $4(>1000)$ & $\begin{array}{c}4 \text { (closest to track } \\
\text { point) }\end{array}$ \\
\hline High & $3(>5)$ & $3(>1)$ & $3(>500)$ & 3 \\
\hline Moderate High & $2(>3)$ & $2(>0.5)$ & $2(>250)$ & 2 \\
\hline Low & $1(>1)$ & $1(0.25)$ & $1(>25)$ & 1 \\
\hline Very Low & & 0 & 0 & 0 \\
\hline
\end{tabular}

\subsection{Results}

The wind swath of Hurricane Matthew covered almost the entire country of Haiti. However, the most intense precipitation occurred in the south western part of the region (Figure 3.3). The epidemic cholera hypothesis suggested that a period of warm temperatures, followed by heavy precipitation, would provide conditions favorable for growth of vibrios in the aquatic environment. Therefore, anomalies of air temperature were calculated. Figure 3.3 shows that the entire region experienced warm temperatures (positive anomalies) during the months of August (Figure 3.4a) and September (Figure 3.4b). Average temperatures for the entire country ranged between $32^{\circ} \mathrm{C}$ to $39^{\circ} \mathrm{C}$, with the north significantly warmer than the southern part of the region. 


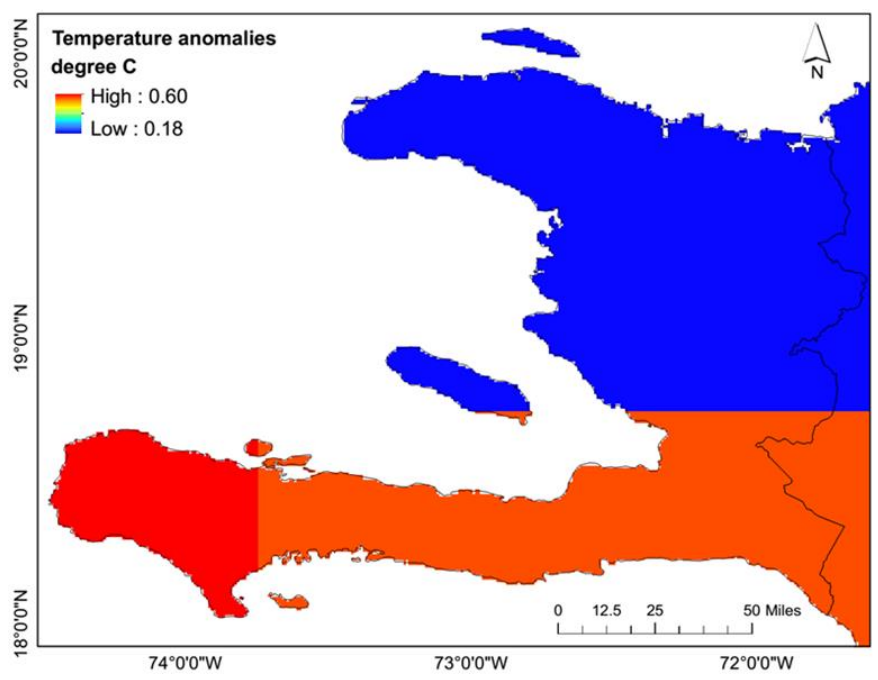

Figure 3.4a: August air temperature anomalies

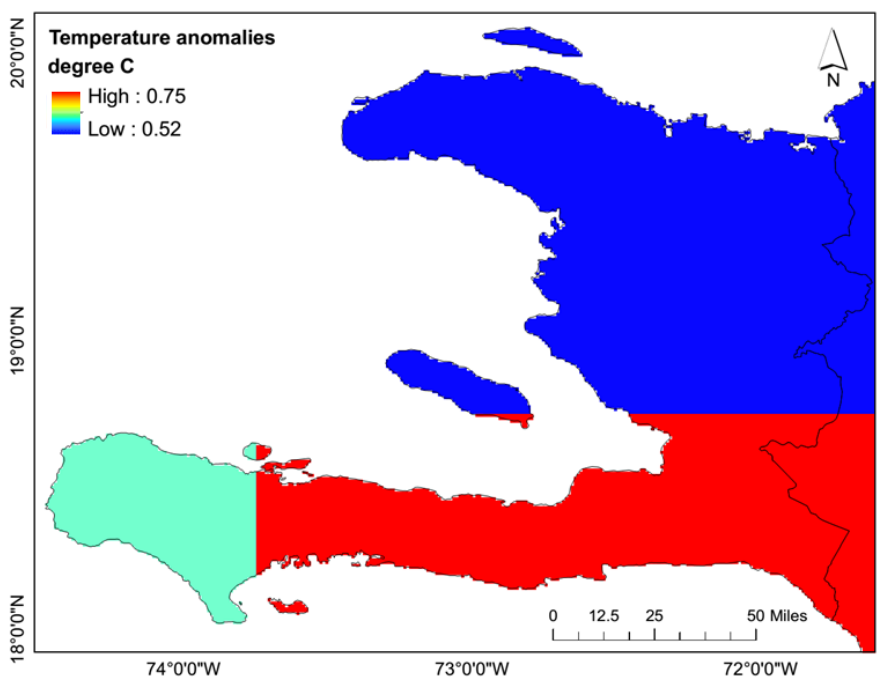

Figure 3.4b: September air temperature anomalies

With prevailing warm temperatures, the region provided conditions optimum for growth of vibrios. Figure 3.5 shows anomalies for precipitation in Haiti during the months of September and October, 2016. Anomalies for the entire month of September (Figure 3.5a) indicate, as shown by the red color, drier conditions (precipitation lower than average), while blue colored regions experienced wetter than the long-term average for the month of September. 


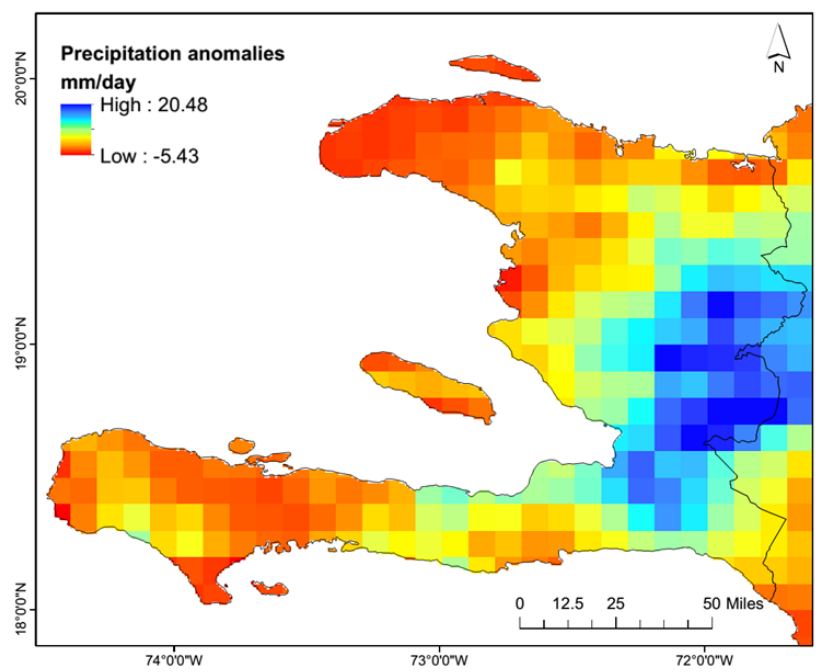

Figure 3.5a: September (1st through 30th) precipitation anomalies.

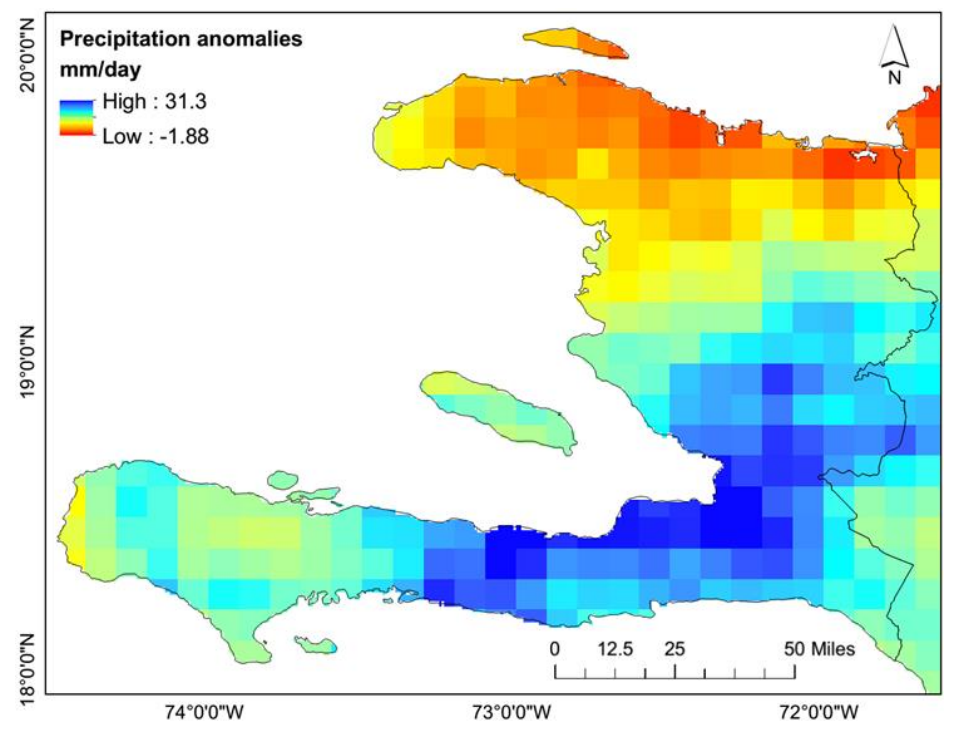

Figure 3.5b: (Sept7-Oct6) precipitation anomalies. 


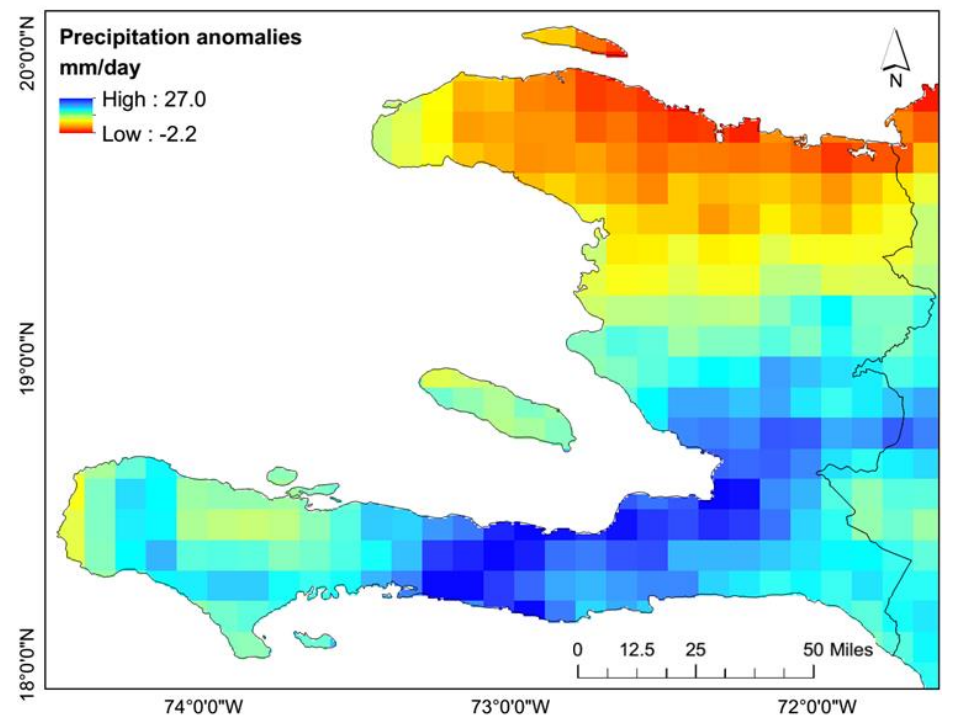

Figure 3.5c: (Sept14-Oct13) precipitation anomalies.

Most of Haiti was dry, except for the middle part of the country. Figure $3.5 b$ shows the month anomaly from September 7 to October 6, 2016, during which time Hurricane Matthew struck the region. The northern parts of Haiti remained dry, while the southern parts were wetter than average for the same duration of time over the last seventeen years. A similar observation was made for the month starting from September 14 and ending on October 13, 2016 (Figure 3.5c).

We used the WRO algorithm to develop cholera risk maps for several months following the hurricane, identifying regions at risk for a cholera outbreak. A pre-hurricane scenario, using August air temperature September precipitation, and normal WASH infrastructure, showed relatively little to no risk of a significant cholera outbreak(risk value less than 0.5 ) for the entire country, except for some regions in the central part of country, where risk of cholera was high (Figure 3.6a). Using the same logic, a cholera risk map for the one-week post hurricane period was developed (Figure 3.6b), showing that the risk of cholera was very high in regions where there was heavy precipitation and high winds of the hurricane damaging an already insufficient WASH infrastructure. 


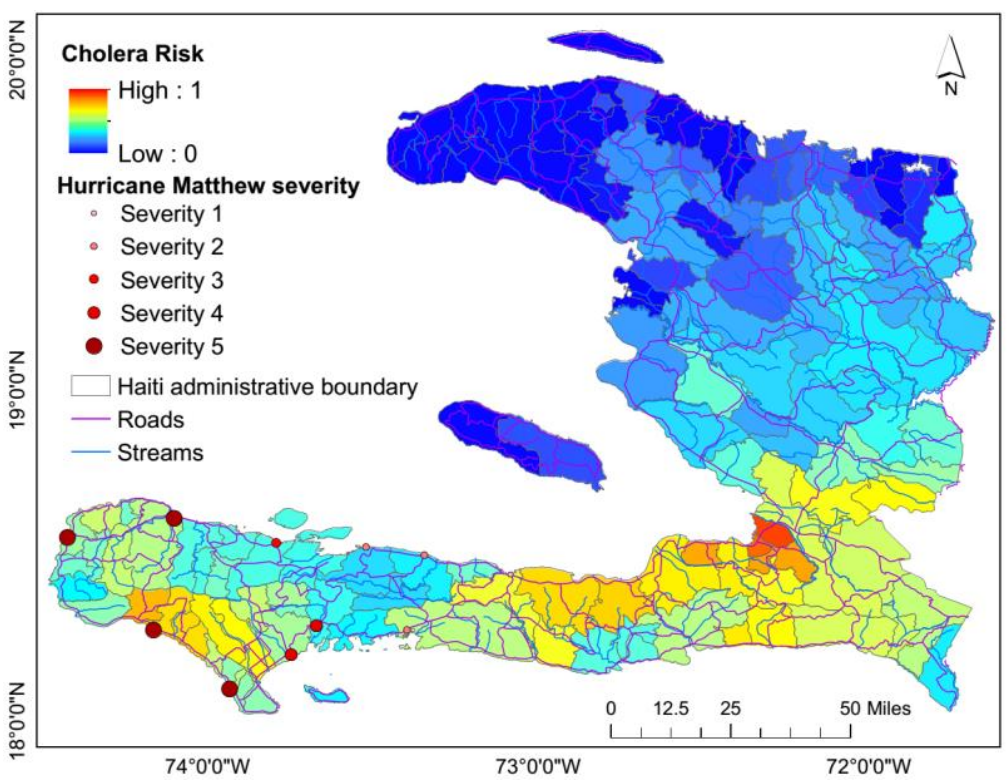

Figure 3.6a: Cholera risk map based on pre hurricane hydro-climatic conditions for the month of September, 2016.

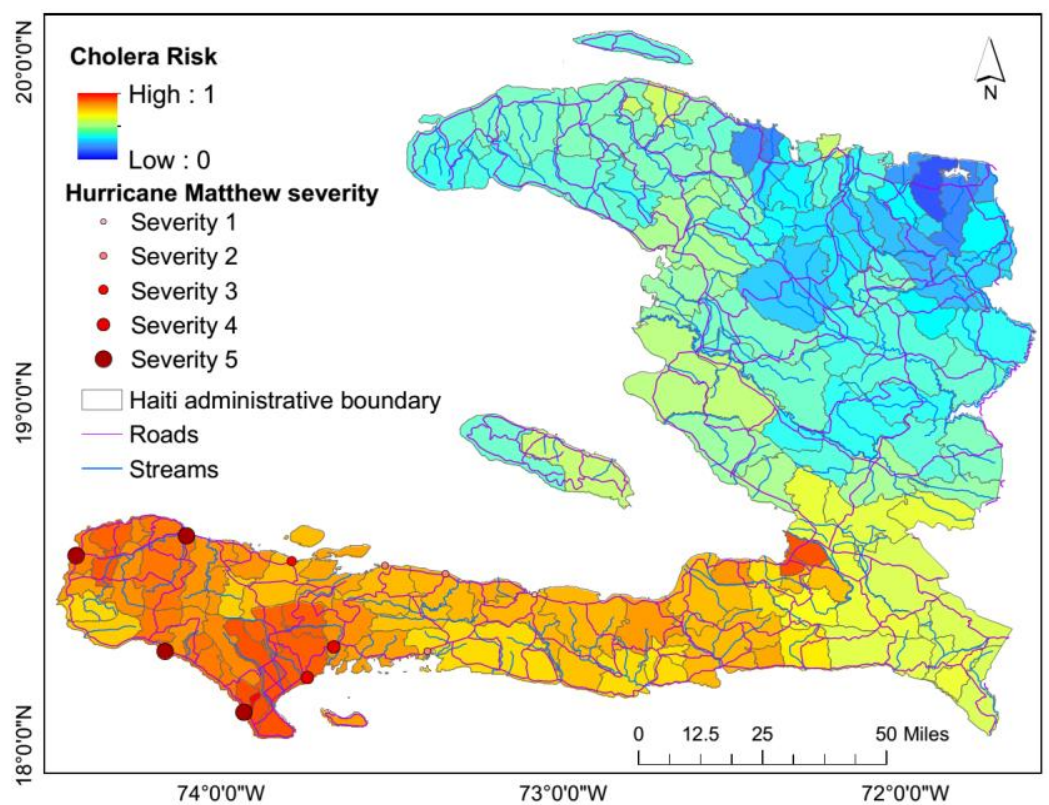

Figure 3.6b: Cholera risk map for the post hurricane period October 07 to November 06, 2016. 


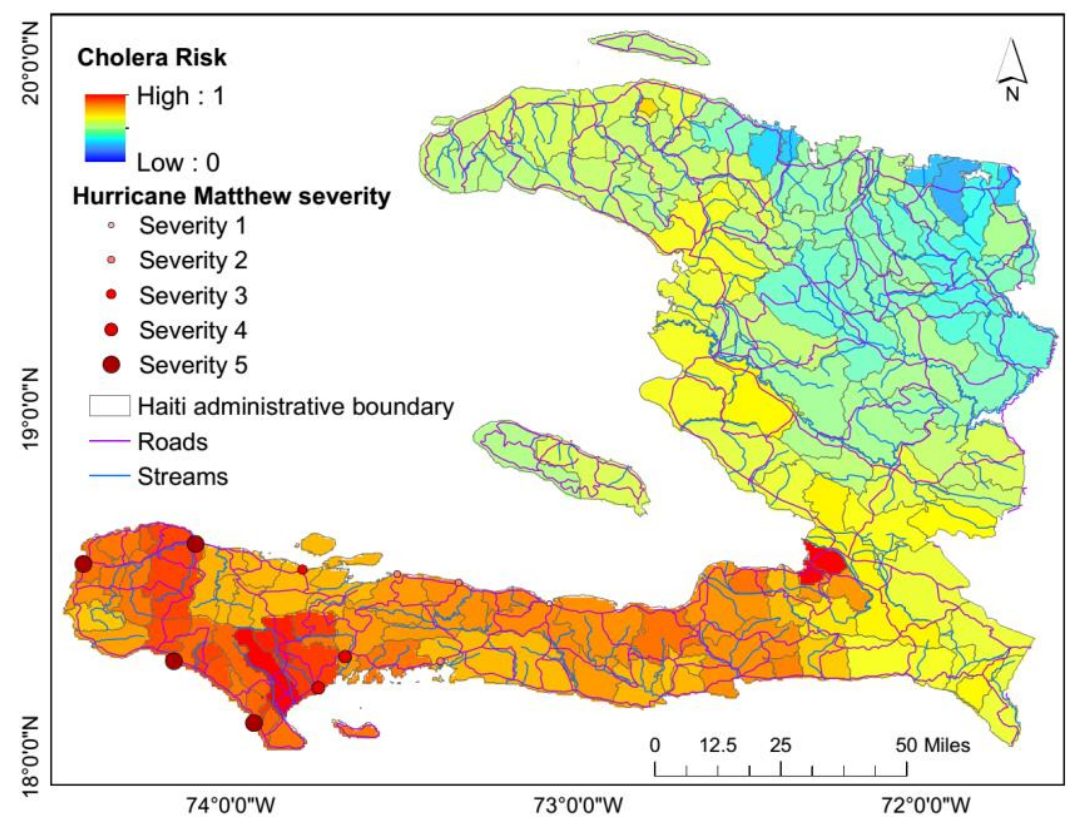

Figure 3.6c: Cholera risk map for the post hurricane period October 14 to November 13, 2016

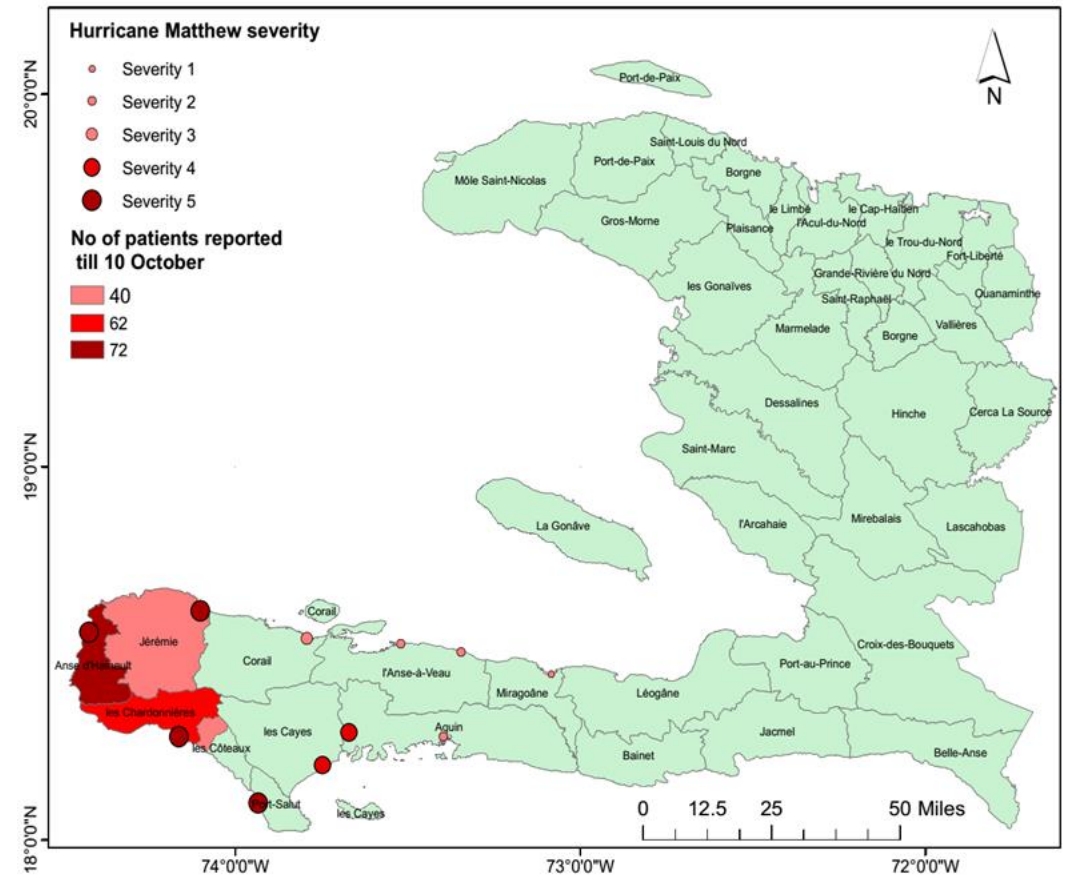

Figure 3.7: Cholera in Haiti up to October 10, 2016. 
The expectation therefore, was that cholera cases could occur within two to four weeks, if the WASH infrastructure remain damaged and insufficient. Dots on the map indicate severity of the hurricane (data extracted from http://resiliencesystem.org/). Further, continuous monitoring of the region, using hydroclimatic parameters allowed production of a risk map for the month of October 14 to November 13, 2016, (Figure 3.6c) the interpretation of which was similar to that taken from Figure 3.6b. As a preliminary validation of the prediction of cholera, Figure 3.7 shows that cholera cases indeed, had begun to occur in the region (up to October $10^{\text {th }}$ ), matching well with regions identified as being within high risk cholera zones (Figure 3.6b). Figure 3.8 shows prediction of cholera for the month of November 2016, for these regions where Hurricane Matthew had struck remaining at risk of cholera, if the WASH infrastructure remained unchanged, i.e. insufficient and damaged.

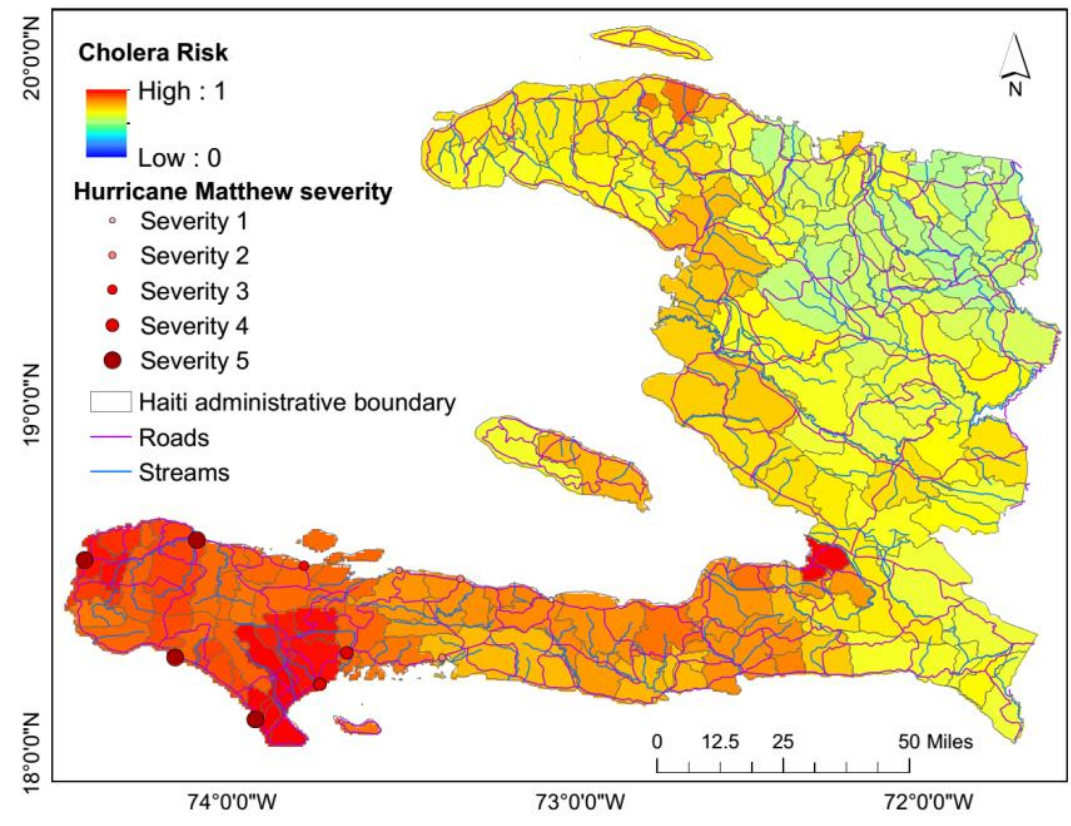

Figure 3.8: Predictive risk map for cholera post hurricane period November 01 to November 30 


\subsection{Discussion}

The advantage of predicting conditions optimal for cholera to occur is an improved capability for the public health system to act in time to prevent cholera, that is, to provide appropriate and timely WASH infrastructure and introduce vaccination for the most vulnerable of the population. There is overwhelming evidence that cholera bacteria are native to the aquatic environment (Colwell $\mathrm{R}$ 1996; Huq et al., 1983; Bartram J, 2008), therefore the probability of weather and regional climatic conditions influencing exposure of the population to pathogenic vibrios in their drinking water is high if WASH conditions are insufficient and/or damaged. Cholera occurs when humans drink water containing the causative agent, V. cholerae, in sufficient concentration to cause illness. There are two schools of thought concerning the route of infection of cholera in human communities. One promotes the hypothesis that the trigger of cholera in the human population occurs only by introduction of infected individual(s) to an unaffected population (Eppinger M et al., 2014). This hypothesis assumes that the bacteria are carried by asymptomatic human(s) capable of contaminating regional water systems, resulting in an explosive prevalence of cholera. The second school of thought divides the cholera outbreak mechanism into two distinct sub-routes. The trigger of the infection is usually through interaction of human populations with the aquatic ecosystem (ponds, rivers etc) containing autochthonous pathogenic vibrios. Transmission of the disease occurs through human-environment-human route, where infected individuals reintroduce the bacteria to the water resource (Codeco $\mathrm{C}$ et al 2008), so that new infections occur from existing water sources and also from person-to-person, when the cases of cholera expand to an outbreak. Our previous results, based on microbiological studies (Hasan N et al., 2012) supported by climatic

analysis of epidemic cholera in several regions of the world (Bangladesh, India, South America and Africa) argue that a cholera epidemic is a complex process, where initial interaction of humans 
with contaminated water from the natural environment triggers the first step of the process, which then leads to an outbreak of disease, supported by secondary transmission routes. Results of this study strengthen the hypothesis that environmental conditions conducive to growth of cholera bacteria in the aquatic environment allow prediction of cholera. Interaction of the human population with the pathogen likely may occur approximately one month in advance of an outbreak or epidemic. Satellite based estimates of precipitation and gridded air temperature data analysis suggest that Haiti is at continued high risk of cholera (Figure 3.7). A few cholera cases have already begun to occur in the south western part of Haiti, validating prediction of cholera in that region based on a "Haiti ministry of public health and population" data (.http://mspp.gouv.ht/site/downloads/Texte\%20cholera\%2010\%20octobre\%202016\%202.pdf). With respect to public health policy, the vulnerable human population of Haiti should be provided with safe water and adequate sanitation facilities. Therefore, the probability of weather and regional climatic conditions enhancing exposure of the population to pathogenic vibrios in the water used as a source of drinking water is high if WASH conditions are insignificant and/or damaged. If there had been strong emphasis on providing WASH infrastructure as a short-term relief to the communities, it would have had a markedly preventative effect following initiation of the cholera epidemic in Haiti during October 2010. In the current scenario, Hurricane Matthew lashed Haiti in October of 2016, and climatic conditions were again favorable for cholera bacteria to become abundant in the aquatic environment, with the greater likelihood of exposure of the population to the disease since both water and sanitation were insufficient. However, as the risk of cholera lessens in the coming months, a sustained effort to rebuild the WASH infrastructure should be undertaken before cholera occurs, especially during the rainy season in 2017 and thereafter. 
While results of our prediction algorithm are promising, some limitations remain, normally the coarse grid representation of the data for air temperature and some latency in availability of precipitation dataset from the Global Precipitation Mission. These limitations do not impact the algorithm directly since contextual interpretation of pixels is employed in the analysis. Lack of field data collection protocols to test for vibrios in the aquatic environment immediately after a natural disaster remain an impediment. Water resources (ponds, rivers etc) should be monitored immediately after a natural disaster in regions of the world where there is insufficient WASH infrastructure. Finally, satellites provide a valuable proxy channel for the development and validation of heuristic prediction algorithms. 


\title{
CHAPTER 04: EVALUATION OF RISK OF CHOLERA AFTER A NATURAL DISASTER: LESSONS LEARNED FROM THE 2015 NEPAL EARTHQUAKE
}

\begin{abstract}
Uncertainty of timing of occurrence and magnitude of natural disasters (such as floods, droughts, earthquakes) affect water resources planning and management in terms of supply of safe drinking water and access to sanitation infrastructure. This in turn has a profound impact on human health. Drinking contaminated water often result in outbreak of diarrheal infections (such as cholera, Shigella etc). Infectious pathogens (such as $V$. cholerae) can survive in aquatic environment under appropriate hydroclimatic conditions. Therefore, the challenge is to estimate the risk of an outbreak of disease after a natural disaster occurs. Using cholera as a signature diarrheal disease and employing the weighted raster overlay method, this paper present a framework for assessing role of water resources, particularly water and sanitation infrastructure (WASH), in determining likelihood of an outbreak of a disease in the human population. The results of this paper indicate that there were favorable hydroclimatic conditions for survival of pathogenic cholera bacteria in natural water systems in the aftermath of the earthquake in Nepal in 2015. However, few cholera patients were reported in the country, indicating that the prevailing resilient WASH infrastructure played a pivotal role in deterring to a disease outbreak.
\end{abstract}




\subsection{Introduction}

Natural disasters can have a significant impact on water resources that include access to and availability of a safe drinking water supply in developing countries. The World Health Organization (WHO) defines natural disaster(s) as "catastrophic events with atmospheric, geologic, and hydrologic origins" (World Health Organization, 2006). Such disasters include earthquakes, volcanic eruptions, storm surges, extreme temperatures, landslides, tsunamis, wildfires, floods, and droughts (EM DAT, 2016). An estimate derived from the International Disaster Database (EM DAT, 2016) suggests that approximately 270 million people are affected annually by natural disasters. Assessment of economic losses by continent, from 1960 to 2014, shows that Asia and the Americas suffer heavy monetary losses from disasters. (EM DAT, 2016). Similarly, a disproportionate number within the human population is affected in Asia, followed by Africa. Floods are perhaps one of the largest subgroups amongst all the disasters (EM DAT, 2016). Overall, an increasing trend is observed in the total number of natural disasters post 1960 arising from enhanced anthropogenic activities, e.g., rapid urbanization, deforestation, and environmental degradation (Leaning and Guha-Sapir, 2013).

Earthquakes, particularly those that are followed by extreme hydroclimatic events such as heavy precipitation, are known to cause massive disruption in the water supply for the human consumption. These often result in outbreak(s) of diarrheal (mainly cholera) infections (Bartlett 2008). From the standpoint of water resource planning and management, it is important to determine how humans interact with water in the immediate aftermath of a disaster and particularly in regions with fragile water, sanitation and hygiene (WASH) infrastructure. Two earthquakes hit Nepal: one on April 25, 2015 with a magnitude of 7.8 and one on May 12, 2015 with a magnitude of 7.3. A massive cholera outbreak following the 2010 earthquake in Haiti made a compelling 
argument for a similar possible situation for Nepal in 2015 (Nelson et al., 2015). However, except for few cases, widespread disease outbreak was not reported in Nepal. The dominant concern of enhanced risk of the cholera in Nepal was associated with devastation of the WASH infrastructure, leading to subsequent exposure of human population to pathogenic bacteria. Once humans are exposed to infective doses of cholera bacteria, transmission of the disease occurs via shedding of pathogens back into the water system, resulting in a widespread outbreak.

Cholera has been classified as occurring in three forms: epidemic, endemic, and mixedmode (Jutla et al., 2015, Jutla et al., 2017). Epidemics are defined as the sudden occurrence of cholera, generally observed in regions distant from the coast. Persistent presence of cholera cases with predictable seasonal outbreak, is termed endemic cholera. Mixed-mode cholera is observed in regions located at the boundary of epidemic and endemic cholera (such as Dhaka, Bangladesh). Human mortality rates of cholera, a fully treatable disease primarily through antibiotics and oral rehydration therapy (Alexander et al., 2013), can be as high as 6\% (Enserink, 2010) in regions affected by natural disasters. Two schools of thought concerning route of infection in human communities have been presented in the literature (details in Jutla et al., 2017). One promotes the trigger of infection in the human population as the introduction of a few infected individual(s) into an unaffected population (Eppinger et al., 2014; Frerichs et al., 2012). This hypothesis assumes that the cholera bacteria are carried by a few asymptomatic individual(s) capable of contaminating the water system (ponds, rivers etc.) such that an explosive prevalence of cholera cases will occur. The second school of thought divides the cholera outbreak mechanism into two distinct sub-routes. The trigger of infection is usually through interaction of the human population with water system containing autochthonous pathogenic vibrios (Alam et al., 2006a; Huq et al., 1983; Jutla et al., 2013; Singleton et al., 1982). Transmission of infection then occurs by a human-environment- 
human route, where infected individuals transmit the bacteria back to the water sources (Codeco et al., 2008; Rinaldo et al., 2014) resulting in new infections. Natural disasters affect the ecology of vibrios, mainly by increasing risk of exposure of the pathogens (Bartlett, 2008) to human population. Congested human communities and refugee camps have a greater risk of cholera, primarily from exposure of that population to naturally occurring vibrios in the aquatic ecosystem (World Health Organization, 2006). It is now well established that a robust water resource, including safe drinking water and appropriate sanitation infrastructure, reduces the risk of cholera (Cairncross et al., 2010).

Environmental factors e.g., precipitation, salinity, temperature, and nutrients have been shown to be associated with presence and growth of cholera bacteria ( $V$. cholerae) in the aquatic environment(Alam et al. 2006b; Epstein 1993; Singleton et al. 1982). Cholera bacteria comprise a component of the commensal flora of zooplankton, and form biofilms on its surface (Colwell 1996; Reidl and Klose, 2002). Copepods, often a dominant component of zooplankton population in the aquatic environment, feed on phytoplankton and a high correlation has been reported between occurrence of copepods and phytoplankton blooms (Huq et al., 1983). With abundance of phytoplankton followed by zooplankton blooms, subsequent increase in the numbers of cholera bacteria in nutrient rich water has been observed in field studies (Alam et al., 2006b) . Outbreaks of cholera over the last several decades in South Asia, Africa, and South America have occurred mostly along coastal areas (Colwell 1996; Constantin de Magny et al., 2008; Jutla et al., 2010). While coastal regions remain the largest natural reservoirs of vibrio bacteria, including $V$. cholerae, epidemiological evidence suggest an increase in cholera incidence in inland regions (Rebaudet et al., 2013). Analysis of the World Health Organization cholera reporting database indicates that almost the entire African continent has reported cholera over the past 20 years, with 
inland regions experiencing massive outbreaks (Jutla et al., 2017). For example, Zimbabwe suffered a cholera outbreak in 2008-2009, with more than 100,000 victims ("WHO | Cholera in Zimbabwe - update" n.d.) seeking medical treatment. Similarly, non-coastal regions of Mozambique, Rwanda, Cameroon and South Sudan reported significant cholera cases in recent decades (Jutla et al. 2015). While there is growing evidence of a relationship between extreme weather events and waterborne infections (Jutla et al., 2017, Jutla et al., 2015, Jutla et al., 2010), prediction of such diseases in the aftermath of a natural disaster have not been rigorously tested.

The goal of this study was to understand impact of natural disaster on occurrence of cholera. Therefore, a key objective was to develop a prediction framework for public health decision-making, relative to reliable safe water supply and access to sanitation infrastructure. To achieve the goal, a hydroclimatological-epidemic cholera hypothesis (Jutla et al., 2013) was employed in Nepal to compare the importance of water resources (WASH infrastructure) during the post-monsoon season following the earthquake in 2015, with respect to the risk of occurrence of cholera. The hydroclimatological-epidemic cholera hypothesis states that a particular region is at high risk of cholera if there is a two month period of warm temperatures (defined as above climatological average air temperature) followed by one month of heavy precipitation (defined as above climatological average monthly precipitation). If these two conditions of warm temperature and heavy precipitation are satisfied and if the integrity of the water resources (WASH infrastructure) is not maintained (having been destroyed in an earthquake), the risk of cholera is predicted as imminent in the region ( Jutla et al., 2013). The working hypothesis of this research, therefore, is that a cholera outbreak did not occur in Nepal in 2015 because one of the conditions of the hydroclimatological-epidemic cholera hypothesis was not satisfied, resulting in limited or no contact by the human population with contaminated water following the earthquake. 


\subsection{Data}

Global Precipitation Climatology Centre (GPCC) gridded monthly precipitation data were obtained from National Oceanic and Atmospheric Administration (NOAA). The precipitation data extend from 1951 to 2016 with a spatial resolution of $0.5^{0} \times 0.5^{0}$. Air temperature data were obtained from NOAA-National Center for Environmental Prediction (NOAA/NCEP). The data were available as monthly mean data from 1948 to 2016 with a spatial resolution of $2.5^{0} \times 2.5^{0}$. Precipitation dataset were regridded to match air temperature dataset. Nepal district boundary, safe drinking water accessibility (\% of people in a district with access to safe drinking water) and accessibility to sanitation facilities (\% of people in a district with access to sanitation) were obtained from "Open data Nepal”, a free source for Nepal ( http://opennepal.net). Earthquake data were obtained from the U.S. Geological Survey (http://earthquake.usgs.gov/earthquakes/map).

\subsection{Methodology}

\section{Spatial hydroclimatic analysis}

In order to apply the hydroclimatological-epidemic cholera hypothesis, monthly anomalies (departure from average condition) for air temperature and precipitation were calculated using Equation 1. For the anomaly estimation for a particular month, an average for 60 years of monthly data was taken and thereafter subtracted from the corresponding month for year 2015 , resulting in a positive or a negative value. A positive anomaly implied that the specific month received greater precipitation than the historical mean condition and vice versa. These were calculated for both datasets, i.e., monthly total precipitation, monthly mean air temperature for all pixels covering Nepal. 
Monthly anomaly $=$ Monthly value Precipitation/Temperature $_{-}$

Long term average value Precipitation/Temperature

... (Equation 1)

\section{Composite weighted raster overlay for risk mapping}

Weighted raster overlay algorithm (Andersson and Mitchell, 2006) was used to produce risk maps

for cholera. Weighted raster overlay is a technique for applying a common measurement scale of values to diverse and dissimilar inputs to create an integrated output with attributable outcomes (e.g., high risk to low risk). A population density layer along with two month's lagged monthly mean air temperature anomaly and one month lagged monthly total precipitation anomaly layers were used to produce the hydroclimatic risk map for likelihood of occurrence of cholera in Nepal. Thereafter, information on regional water resources (WASH infrastructure) was added to the risk assessment. For post-earthquake risk assessment, weights for the WASH layer were reduced using the earthquake intensity, with the assumption that significant damage to the infrastructure occurred in the region.

When applying the weighted raster overlay algorithm (Andersson and Mitchell, 2006), all input raster layers must have an assigned integer value, or it must be converted to an integer. Each input raster is assigned a new value based on an evaluation scale. The new values were deemed to be "reclassification" of the original input raster values. The evaluation scale was determined based on the range of all raster layers for the variable under consideration. For example, the air temperature anomaly evaluation scale was determined based on maximum and minimum values of the raster layers for all May, June, July anomalies. Every input raster was weighted according to importance (in terms of percent influence) and was converted to relative percentage; total being 
100 (see Table 4.1). Changing evaluation scales or percent influence can change the results in the final risk map. Different weights were computed (Table 4.1, 4.2) while determining risk of cholera under various scenarios e.g. hydroclimatic (Table 4.1) and WASH based risk (Table 4.2) assessment.

Table 4.1: Hydro-climatological risk weights used in cholera outbreak risk clustering

\begin{tabular}{lcccc}
\hline \multicolumn{5}{c}{ Hydro - climatological risks (\%) } \\
\hline Risk & Precipitation & Temperature & Population & Cholera \\
level & anomalies & anomalies & densities & outbreak Risk \\
\hline Very & 36 & 36 & 27 & 100 \\
High & 27 & 27 & 18 & 73 \\
\hline High & 18 & 18 & 9 & 45 \\
\hline Moderate & & & & 9 \\
High & 0 & 9 & 0 & 0 \\
\hline Low & & 0 & & \\
\hline Very Low & & & & \\
\hline
\end{tabular}

The relative weight of each variable was assigned a risk level, e.g. very high, high, moderate high, low, or very low. Hydroclimatological departure from normal conditions was assumed to the strongest contributor to risk of cholera. Using each pair of precipitation and temperature anomalies, along with population density, three composite maps of spatial cholera risk for July, August and September were generated. 
Table 4.2: Socio-hydro-climatological risk weights used in cholera outbreak risk clustering

$$
\text { Socio - Hydro - climatological risks (\%) }
$$

\begin{tabular}{lcccccc}
\hline Risk & $\begin{array}{c}\text { Precipitation } \\
\text { anomalies }\end{array}$ & $\begin{array}{c}\text { Temperature } \\
\text { anomalies }\end{array}$ & $\begin{array}{c}\text { Population } \\
\text { densities }\end{array}$ & $\begin{array}{c}\text { Safe water } \\
\text { accessibility }\end{array}$ & $\begin{array}{c}\text { Sanitation } \\
\text { services }\end{array}$ & $\begin{array}{c}\text { Cholera } \\
\text { outbreak Risk }\end{array}$ \\
\hline Very & 21 & 21 & 16 & 21 & 21 & 100 \\
High & 16 & 16 & 11 & 16 & 16 & 74 \\
\hline High & 11 & 11 & 5 & 11 & 11 & 47 \\
\hline Moderate & & & & & \\
High & 0 & 5 & 0 & 5 & 0 & 0 \\
\hline Low & 11 & & & & \\
\hline Very Low & & 0 & & & & \\
\hline
\end{tabular}

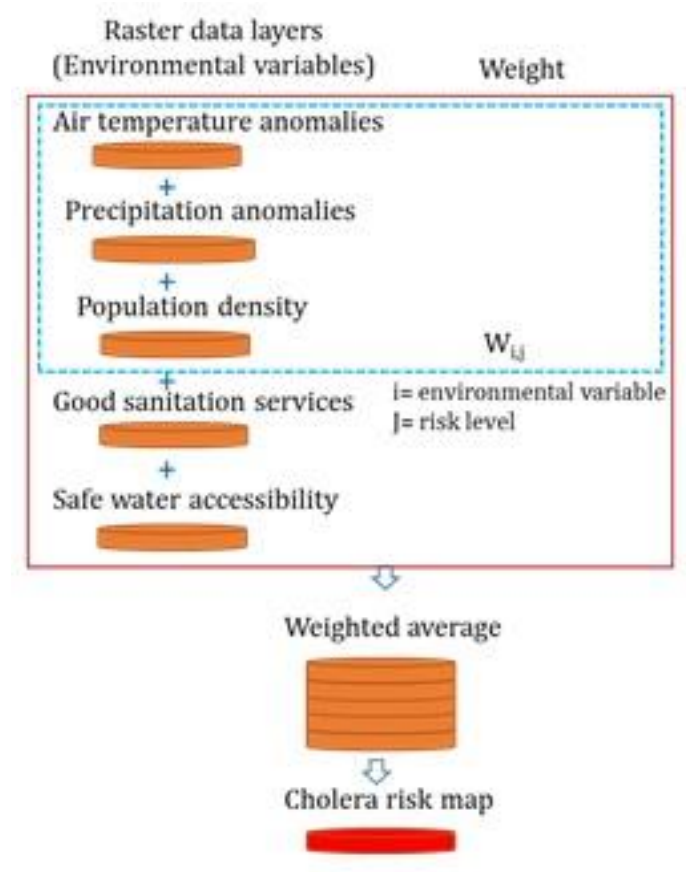

Figure 4.1: Weighted raster overlay, with blue dotted box representing layers used to generate the hydroclimatic risks map and solid red box incorporating water resources into the risk calculations. 
Figure 4.1 shows the flowchart for implementation of the weighted raster overlay algorithm. The blue dotted box at the left represents layers used to generate the hydro-climatic risk map and the solid red box incorporates the WASH infrastructure into the risk computation.

\subsection{Results}

\subsubsection{Hydroclimatological risk of cholera}

By incorporating the theoretical pathway proposed in a previous publication (Jutla et al., 2015) for hydroclimatic epidemic cholera hypothesis, it was possible to calculate favorability of conditions for survival of pathogenic vibrios. Using gridded precipitation and temperature data, monthly anomalies were calculated for a two month lead time for air temperature and one month lead time for precipitation for the entire country of Nepal (Figure 4.2). May to July, 2015 air temperature and June to August precipitation data were selected for computation of anomalies to examine hydroclimatic risk of cholera (from July to September, 2015).

Both precipitation and air temperature anomalies were calculated for selected months for each pixel and averaged over 75 districts to capture spatial and temporal variability across entire country. Red color represents warm temperature (positive anomalies- Figure $4.2 \mathrm{a}, \mathrm{b}$ and c). On the other hand, red color represents decreased precipitation (Figure 4.2d, e, and f: negative anomalies). Temperature anomalies (Figure 4.2a, b, c) suggest an overall warm condition across Nepal in 2015, compared with several previous years. Months of May (Figure 4.2a) and July (Figure 4.2c) showed very high positive departures notably in the central and western parts of Nepal. A maximum of $2^{0}$ increase in temperature was observed during May 2015, in western Nepal. Air temperature for selected months, in eastern Nepal was mostly recorded at the long term average in 2015 with limited spatial and temporal variability in the entire region. 


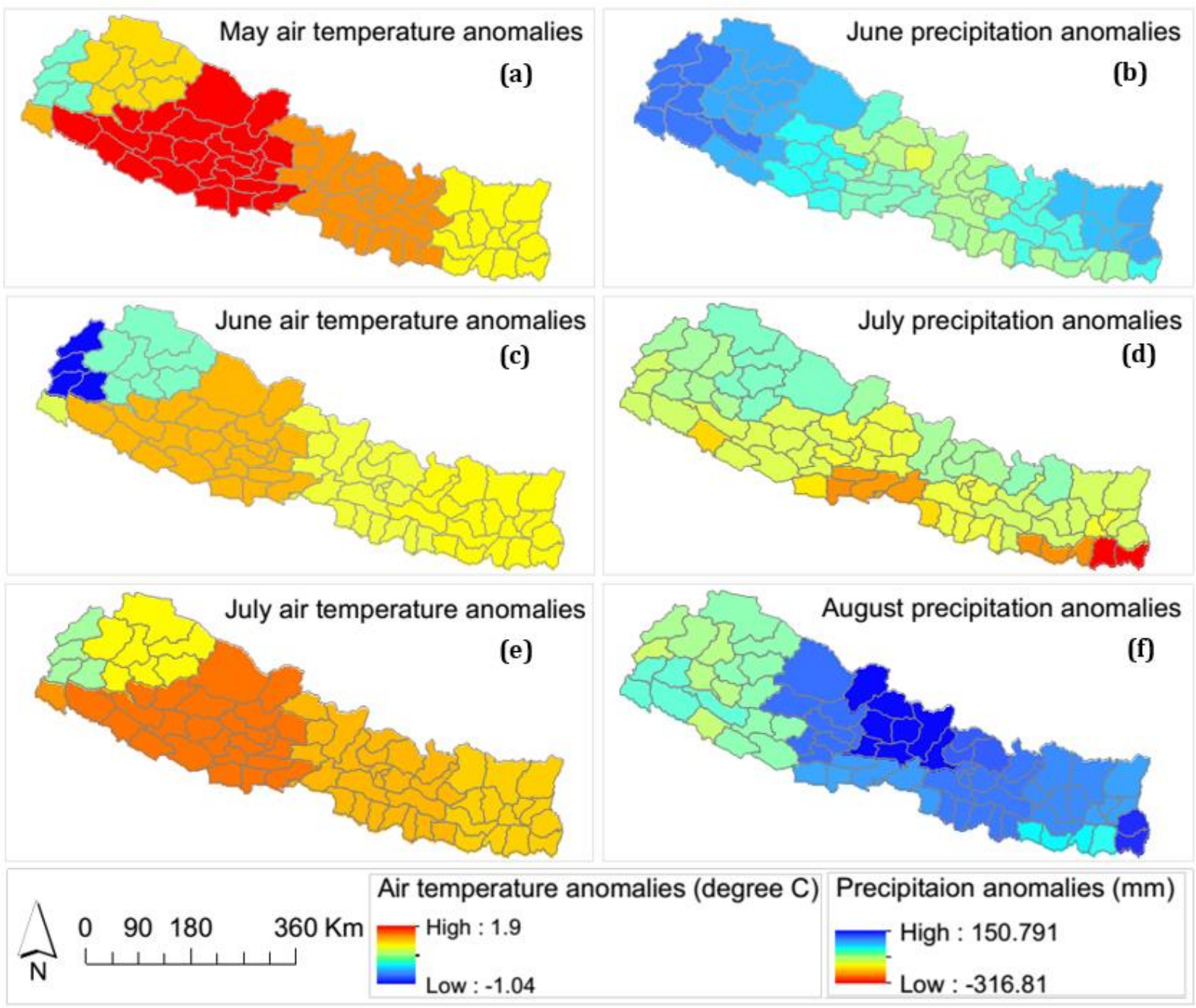

Figure 4.2: Hydroclimatological anomalies for May through August, 2015

Monsoon season precipitation (Figure $4.2 \mathrm{~d}$, e, f) anomalies in 2015 were as high as $150 \mathrm{~mm}$ in the north central region. The month of August was wet, compared to long term precipitation for the region. During June of 2015, western Nepal showed positive anomalies indicating wet conditions when compared with the long term average of precipitation. In contrast, July precipitation indicated a negative precipitation anomaly (dry conditions) across Nepal. Therefore, even with warm temperatures present during June, the hydroclimatological risks of cholera remained low. 
The weighted raster overlay algorithm was then used to superimpose air temperature, precipitation, and population layers to generate a single composite spatial map of the risk of occurrence of cholera. Coupled anomalies of May air temperature-June precipitation; June air temperature-July precipitation; July air temperature-August precipitation were used to compute the hydroclimatological risk of cholera for the months of July, August, and September respectively. The spatial cholera risk map (Figure 4.3) for July, August, and September indicate high cholera risk in western Nepal during July and in the central to eastern part of Nepal during September.

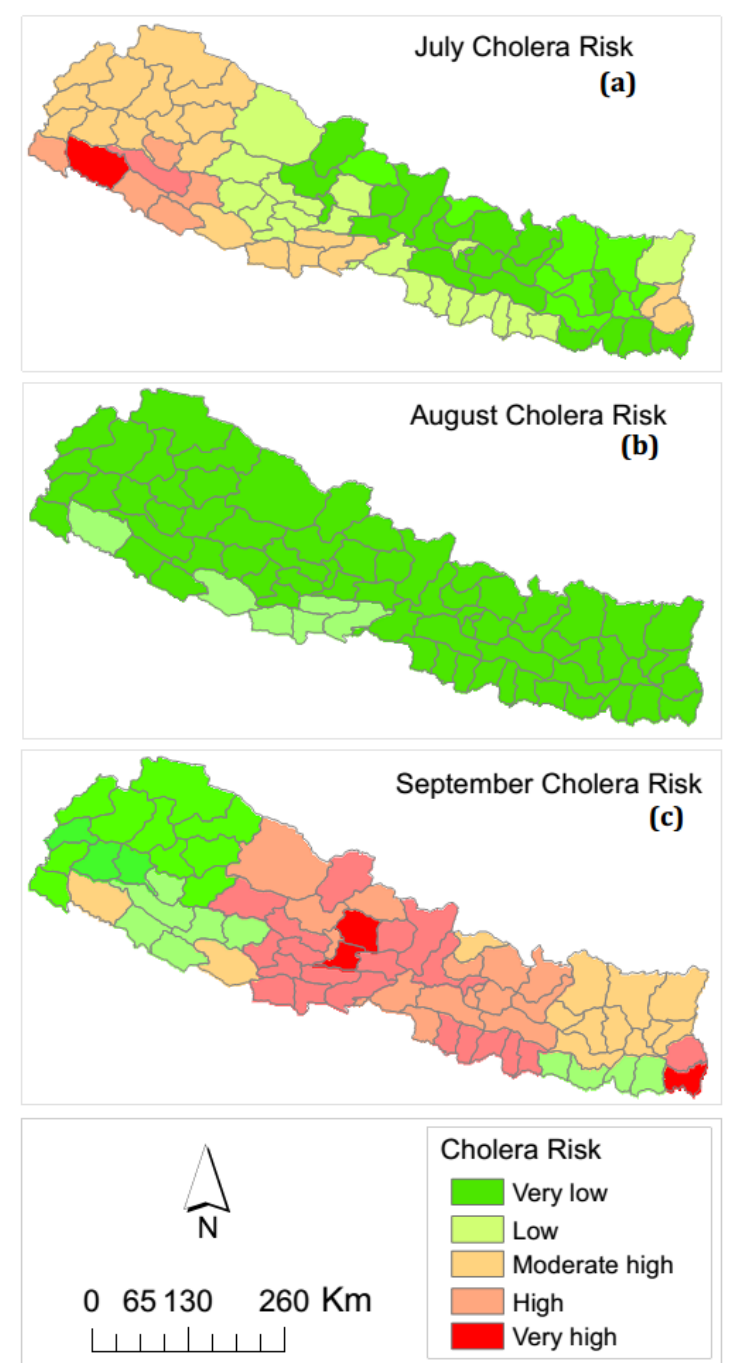

Figure 4.3. Cholera risk map based on hydroclimatology of the region 
The month of August indicates cholera risk free throughout Nepal, since hydroclimatological anomalies (temperature and precipitation) did not indicate sufficient deviation toward conditions conducive for an outbreak of cholera.

\subsubsection{Integrated WASH and hydroclimatological risk}

Cholera is transmitted by drinking water contaminated with infective doses of the pathogenic vibrios, especially when the water resources are compromised, leading to enhanced interaction of humans with the pathogen. Therefore, in addition to presence of a susceptible human population, the availability of a WASH infrastructure must be taken into account to calculate a realistic risk of cholera in a region. Access to safe water and sanitation facilities in Nepal are characterized by high spatial variability (Figure 4.4).
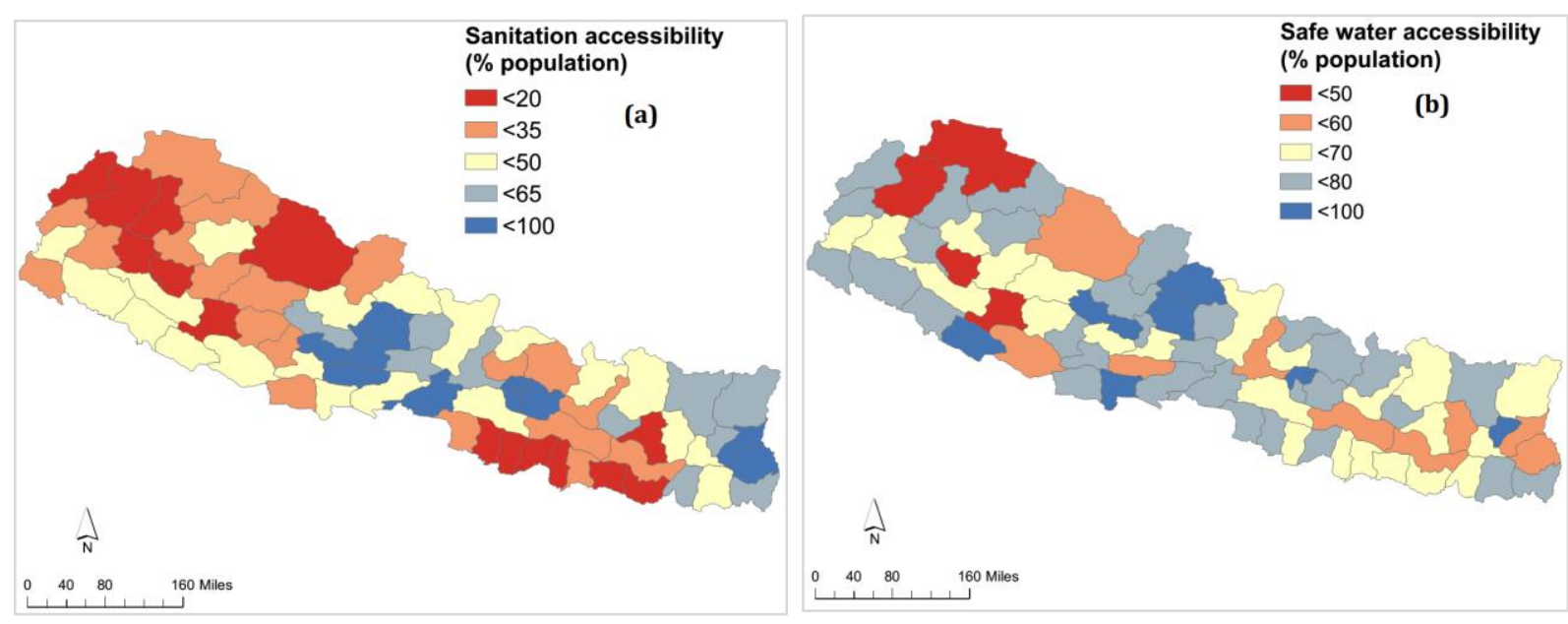

Figure 4.4. Status of water resources (a) sanitation accessibility and (b) safe water accessibility in Nepal

The capital, Kathmandu, and surrounding areas have a relatively better quality of WASH infrastructure than the rest of the country. Sanitation in western and south-eastern Nepal is generally considered to be lacking. 


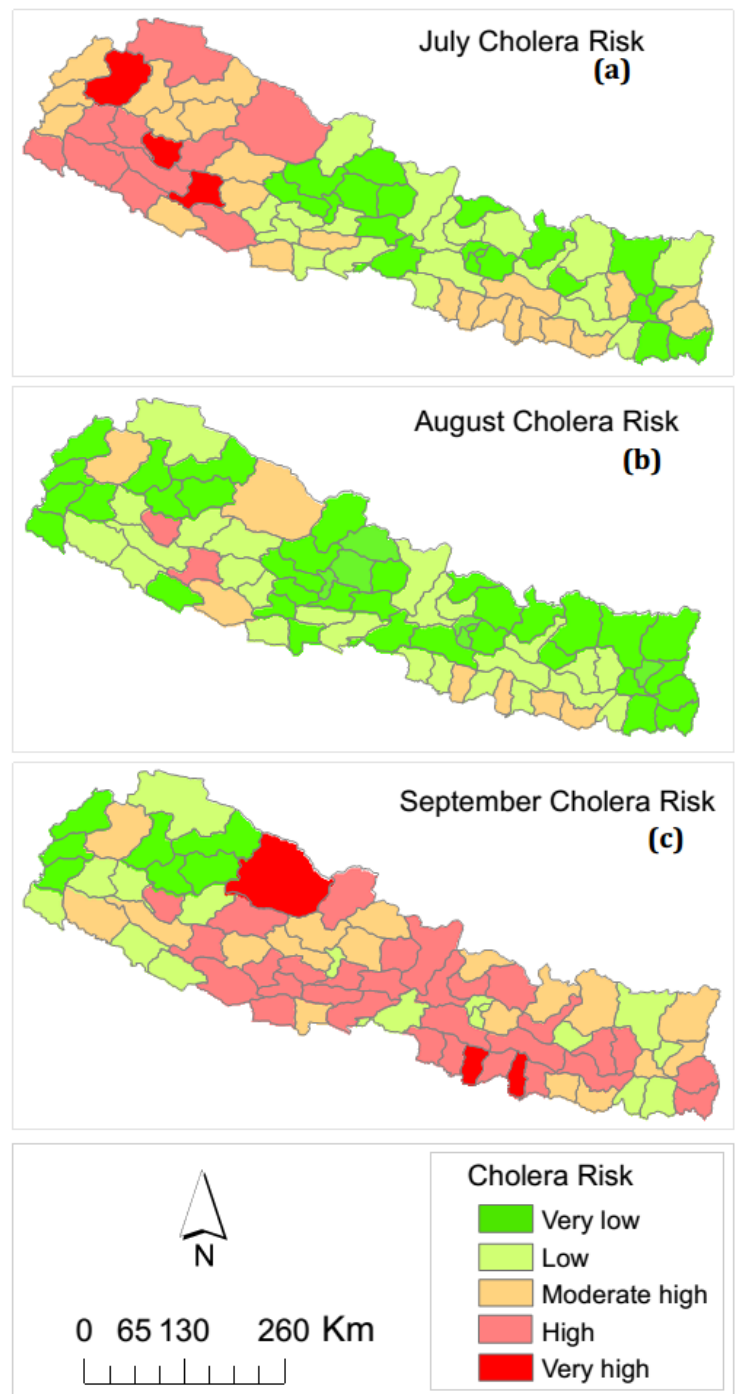

Figure 4.5. Cholera risk map post-earthquake with resilient (present or intact) water resources and hydroclimatology

Therefore, WASH was included in the analysis with the assumption that there was no impact of the earthquake on the infrastructure. After application of the composite overlay algorithm, cholera risk maps were generated (Figure 4.5) for July (Figure 4.5a), August (Figure 4.5b), and September (Figure 4.5c). Figure 4.5 shows those areas in and around the city of Kathmandu, most severely impacted by the earthquake, did not have a high risk of cholera assuming water and sanitation resources were relatively unaffected by the earthquake. Without an earthquake, the risk of cholera 
for southeastern Nepal was high, indicated by hydroclimatic conditions and available WASH infrastructure.

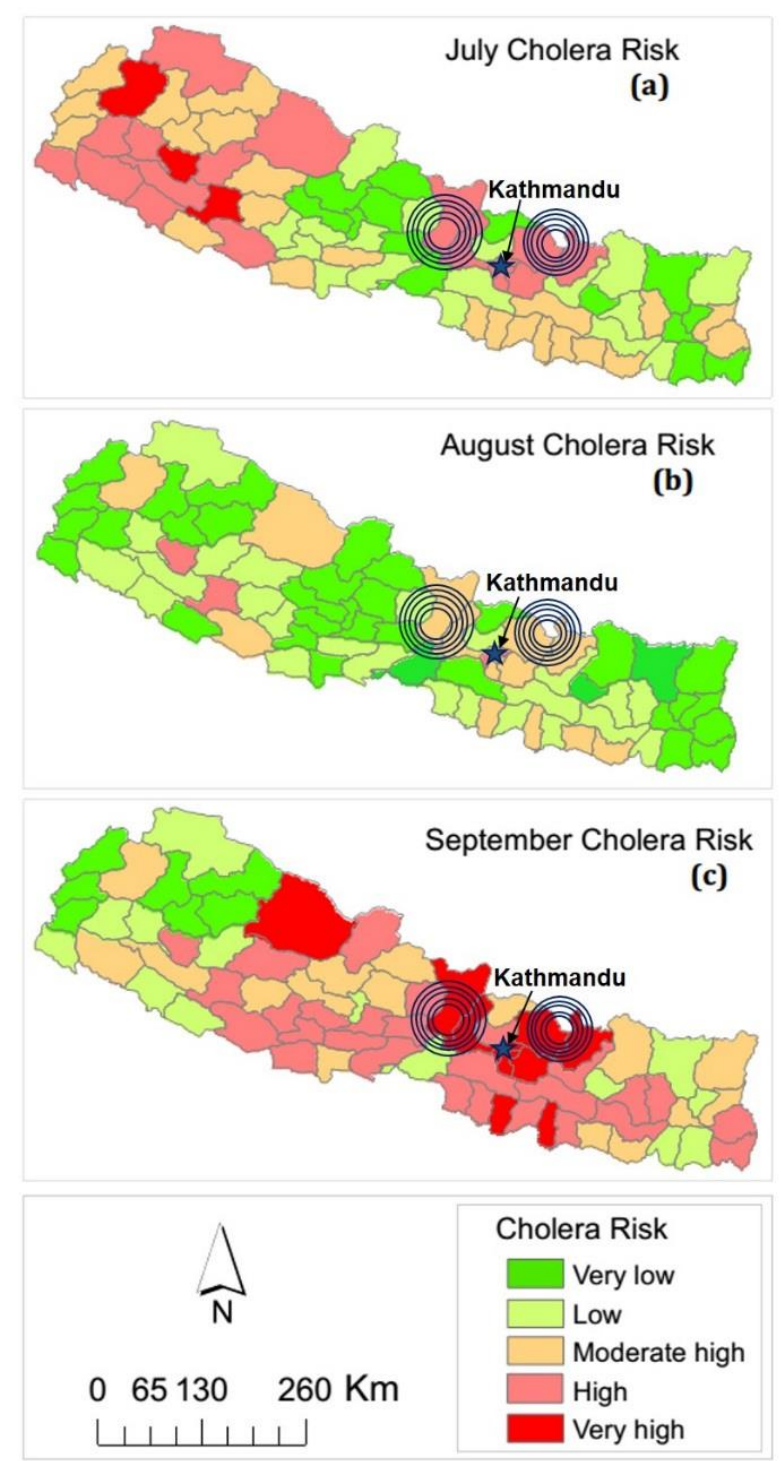

Figure 4.6. Cholera risk map post-earthquake including a devastated water supply after the earthquake (circles show extent of the earthquake).

A challenge after any natural disaster is to determine influence of devastated WASH infrastructure for the human population with respect to potential for an outbreak of a disease. Therefore, another scenario was generated that included a broken WASH infrastructure, with expectation of a deteriorated water resources and increased risk of cholera in those regions where 
hydroclimatic conditions were favorable for bacterial growth. Using an earthquake magnitude above 6.0 as a benchmark for the most severely impacted region and complete collapse of the water resources, at the epicenter (Kathmandu and nearby cities) and surrounding districts, a new cholera risk map was generated (Figure 4.6). The cholera risk prediction for July (Figure 4.6a) and August (Figure 4.6b) was low but for September (Figure 4.6c) it was very high for the earthquake affected zones.

\subsection{Discussion and Conclusion}

The objective of this study was to understand the relationship of water resources (WASH) and public health, with reference to the risk of cholera outbreak in the aftermath of an earthquake in Nepal in 2015. Using the weighted raster overlay method, and previously developed hypothesis for epidemic cholera (Jutla et al., 2013, 2015), we have developed a schema to integrate information on disaster with existing water resources as well as hydroclimatic processes to estimate the risk of cholera in the region. An important outcome of this study, apart from validating the epidemic hypothesis, was that an outbreak of cholera caused by inferior or damaged water resources would have led to an outbreak of cholera in the immediate aftermath of the earthquake, assuming total collapse of the water resources for the region and without external aid provided to human population. However, a widespread cholera outbreak was not reported in Nepal, ascribable to the fact that the need for a WASH infrastructure was immediately strengthened following the earthquake. Application of the weighted raster overlay method (Andersson and Mitchell 2006; Goyal et al., 2015; Nasrollahi et al., 2017) was able to compute the risk of cholera and provided an intuitive assessment of the effect of WASH infrastructure on an outbreak of cholera.

It is important to note that some cholera cases were reported in Kathmandu and adjoining areas after the earthquake (EDCD, 2015; The Himalayan Times, 2015). While the epidemic 
hypothesis proved valid in capturing risk of cholera, yet a widespread outbreak of infection did not occur in the region, despite hydroclimatic conditions favorable for growth of vibrios. Two mechanisms for cholera epidemic are: trigger and transmission. A trigger for cholera occurs with interaction of humans and contaminated water resources. However, for a widespread cholera epidemic to occur in human population, appropriate transmission mechanisms are required. If the trigger of cholera is controlled, transmission of cholera within the human population is averted. In Nepal after 2015 earthquake, several aid agencies including Nepal Government Epidemiology and Disease Control Division (EDCD, 2015) and WaterAid (WaterAid, 2015), mobilized resources quickly and efficiently, limiting interaction of the population with the damaged WASH infrastructure. An aggressive educational campaign was also launched in the Kathmandu region (EDCD, 2015; UNICEF, 2015) and safe drinking water and sanitation facilities were quickly provided. UNICEF provided sustained WASH facilities to about 1.3 million people up to nine months after the earthquake (UNICEF, 2015). Relief efforts in Nepal provided safe and sustained WASH access, successfully averting a cholera epidemic. These actions were key to mitigating the impact of the earthquake, compared to Haiti where a massive cholera epidemic occurred in 2010. During the aftermath of Haiti earthquake in 2010, efforts to provide WASH infrastructure were relatively limited and hence a massive outbreak was reported following favorable environmental conditions for supporting growth of vibrios in water (Eisenberg et al., 2013; Jutla et al., 2013). Data on WASH availability after natural disasters is not routinely collected, although efforts are made to provide access to safe drinking water. A systematic review (Handzel et al., 2013) of about eighteen experiments, in several regions of the world, on WASH intervention for controlling cholera concluded that a robust WASH infrastructure generally aids in averting spread of cholera infection in human population. Similarly, a controlled experiment conducted in Bangladesh (Khan 
and Shahidullah, 1982) suggested that elimination of cholera is not possible, but prevalence of disease is reduced if appropriate WASH infrastructure was provided. These findings corroborate with our observations that providing WASH infrastructure was perhaps one of the important activities that helped forestalling cholera outbreak in Nepal.

Post-earthquake conditions in Nepal have been compared with those following the Haiti earthquake in 2010 (Auerbach, 2015). The absence of cholera epidemic in Nepal provided an evidence for the importance of resilient and operable WASH infrastructure coupled with effective regional education concerning safe water use. An important lesson, from the perspective of water resource planning and management, is that, in addition to vaccination protocols, provision of WASH infrastructure in the immediate aftermath of a natural disaster is critical. Reliable operation of safe water and sanitation resources after a natural disaster are key factors to prevent water-borne diseases, notably cholera. Nepal earthquake provides an excellent example where, despite conducive environmental conditions for growth of vibrios, a cholera epidemic did not occur. Public educational campaigns on disaster preparedness [such as "drop, cover, and hold on," (Auerbach, 2015) must be accompanied with safe water and sanitation protocols (Showstack, 2015). From the standpoint of water resource managers, it is critical that public health outcomes must be integrated with the infrastructure planning for a sustainable solution(s) to avoid disease outbreak. The results of our research are promising. Application of our proposed framework will allow prediction of timing and location for cholera, and inform WASH related public health decision making for water resources planning and management for disaster relief efforts. . 


\title{
CHAPTER 05: EXPLORATORY ANALYSIS OF PATHOGENIC VIBRIOS IN COASTAL WATER
}

\begin{abstract}
Several coastal regions have documented presence of several vibrios with characteristic climatic signatures. But what is the extent and spatial variability of risks of vibrio growth along the coast in the world and how this spatial pattern shows variability to lead reemergence of vibrios at new locations, thus threatening more human populations? Also, the occurrence of Vibrio in marine environments are greatly impacted by environmental conditions and therefore, can pose a significant threat to various countries and cultures. In this study, a three year samples of water, oyster, and sediment were collected from two sample sites in the Chesapeake Bay (Chester River and Tangier Sound) and the total and pathogenic forms of $V$. vulnificus and $V$. parahaemolyticus were determined. In both the Chester River and Tangier Sound, the two Vibrio spp. were present in higher abundance during summer and fall months; with $V$. vulnificus peaking during July and October and V. parahaemolyticus in July and August. V. vulnificus was found to be present in significantly higher numbers in all sample types at both sites. Results showed that chlorophyll-a, dissolved oxygen, and water temperature were significantly associated with Vibrio spp. density and incidence of $V$. vulnificus was additionally associated with turbidity and salinity. Thereafter, a multi sensor ensemble niche modeling approach was adopted to model the emergence of $V$. cholerae using sea surface temperature, salinity, chlorophyll, and bathymetry data along coastal regions. The model was calibrated on a global scale first based on previously reported coastal occurrences and then simulated along the coast of entire globe.
\end{abstract}

\subsection{Introduction}


Marine and estuarine Vibrio spp., notably V. vulnificus and V. parahaemolyticus, have long been established as native to the aquatic environment and present in the water column, sediment, and in filter feeders, e.g., shellfish (Vezzulli et al., 2010; Pruzzo et al., 2008; Colwell, 1996). In the United States, Vibrio parahaemolyticus is responsible for approximately $1 \%$ of all food borne illnesses and is the leading cause of bacterial gastroenteritis associated with seafood consumption (Chen et al., 2017). Vibrio vulnificus can cause primary septicemia associated with raw oyster consumption and also severe wound infections when individuals are exposed to contaminated seafood or seawater. According to Center for Diseases Control (CDC), Vibrio vulnificus and Vibrio parahaemolyticus are responsible for approximately 8,000 food borne and non-food borne infections per year in the United States, with the majority occurring during the warmer months of the year when environmental conditions are favorable for their growth.

Vibrio parahaemolyticus produces three types of specific hemolysins, thermostable direct hemolysin $(t d h)$, tdh-related hemolysin $(t r h)$, and thermolabile hemolysin $(t h)$. Of these three hemolysins, both $t d h$ and $t r h$ have been shown to be associated with the pathogenic form of Vibrio parahaemolyticus, infection with which results in gastroenteritis in humans. Interestingly, th, is used as a marker to measure the total number of pathogenic $V$. parahaemolyticus in a sample (Kishishita, et al., 1992., Nishibuchi et al., 1995) and the hemolysin gene, $v v h$, employed to detect the total number of $V$. vulnificus in a sample (Johnson et al., 2010, Wright et al., 2010).

Vibrios spp. have been isolated from many different aquatic organisms ranging from birds, fish, shrimp, and seaweed to filter feeders, such as mussels and oysters. Most seafood require processing, such as cooking or freezing, before consumption. Oysters are unique in that they are commonly consumed raw and the oyster industry worldwide is large, with a very extensive market 
for oysters. Seafood safety and hygiene, especially for oysters, receive significant attention, notably with respect to pathogenic microorganisms. Because oysters are filter feeders, they are able to concentrate bacteria, especially Vibrio spp., to such an extent that the number of bacteria in oysters will be much higher and up to 100 times than in the surrounding water (Froelich, and Noble, 2016). V. vulnificus is believed to be commensal to oysters, that is, to exist alongside, or more specifically, inside oysters (Oliver, 1989; Tamplin and Caspers et al., 1992, Iwamoto et al., 2010). The oyster therefore often acts either as a reservoir for Vibrio bacteria, serving as a protective niche when environmental conditions become harsh, e.g., winter temperatures, or as "passive concentrators," artificially raising the concentration of Vibrio spp. by extracting them from the water column (Vezzulli et al., 2010, Wright et al., 1993). One of the major concerns with these bacteria is the increase in cases of Vibrio related diseases in the United States reported by health authorities, including CDC (Newton et al., 2012, Iwamoto et al., 2010). The diseases reported are often those associated with consumption of raw or uncooked seafood and frequent each source of infection is oysters (Slayton et al., 2014; Wallace et al., 1999; Wechsler et al., 1999). This association most likely is reflective of the fact that mollusks often act as reservoirs for the bacteria, with Vibrio spp. frequently detected in freshly harvested oyster meat and shellstock (Slayton et al., 2014, Cook et al., 2002, DePaola et al., 2003).

The human health effect risk that Vibrio spp. pose is the most serious impact of the relationship of Vibrio spp. with mollusks. Vibrio vulnificus, for instance, has a $50 \%$ mortality rate when infection with V. vulnificus develops into septicemia (CDC, 2016, Bross et al., 2007, Hilton T et al., 2006). The threat of a $V$. vulnificus illness is associated with the unsightly and dangerous tissue damage that occurs if an abrasion or open wound is exposed to contaminated water. 
Ingestion of oysters containing V. parahaemolyticus can lead to severe gastroenteritis (Bross et al., 2007; Chatzidaki-Livanis et al., 2006).

In addition to water and shellfish, sediment has also been identified as a reservoir for $V$. parahaemolyticus. It has been well documented that in sediment the overall population of Vibrio spp. can be concentrated. V. parahaemolyticus associated with sediment could survive near freezing temperatures in the Chesapeake Bay during the winter months (Kaneko and Colwell, 1973). Similarly, on the West Coast of the U.S. in Washington State, evidence was obtained showing detection numbers of $V$. parahaemolyticus in sediment (Baross et al., 1970). The concentration of $V$. parahaemolyticus are higher in sediment than that of $V$. vulnificus, whereas the opposite is the case for oysters and their surrounding water, indicating V. parahaemolyticus survives well in a sediment rich environment (Johnson et al., 2010). This is an important observation since large numbers of $V$. parahaemolyticus can be found in sediment, with the potential for reintroduction into the water column when sediment is disturbed by currents or mechanical action (Johnson et al., 2010).

Understanding the environmental influence on distribution and growth of Vibrio spp. is important, especially in regard to seasonality. Several studies have successfully linked the presence of Vibrio spp. and Vibrio epidemiology with predictable patterns related to changing seasons in the Chesapeake Bay and globally (Paranjpy et al., 2015).

The Chesapeake Bay is an estuarine environment where salinity and nutrients vary depending on the influx of freshwater (Banakar et al., 2011). Previous studies have shown that $V$. vulnificus can be detected over a very wide range of temperature, from $8^{\circ} \mathrm{C}$ to $26^{\circ} \mathrm{C}$ (Wright et al., 1996). During colder months of the year when the temperature range is both lower and narrow in range, $V$. vulnificus is either not detected or detected only by employing molecular probes rather 
than culture, indicating a seasonality of $V$. vulnificus in the Chesapeake Bay, as reported in very early work on V. parahaemolyticus (Wright et al., 1996; Kaneko and Colwell, 1973; Kaneko and Colwell, 1975). For Vibrio parahaemolyticus, the minimum growth temperature is near or above $17^{\circ} \mathrm{C}$ and salinity near or below 13 parts per thousan (ppt) (Kelly and Stroh 1988; Kelly 1982). $\mathrm{V}$. vulnificus does not grow at salinities above 25 ppt with an optimal temperature of growth near 37 ${ }^{\circ} \mathrm{C}$ (Kelly 1982; Motes et al., 1988; Kaspar and Tamplin, 1993; Drake et al., 2007). In addition to physical and chemical variables associated with growth of Vibrio spp. other factors such as pressure influence growth of these bacteria, as it has been shown that at greater depths in the water column, there is a statistically significant increase in the number of $V$. vulnificus (Wetz et al., 2014; Wise et al., 1996).

This reseach conducted a comprehensive study in Chesapeake Bay for both detection and exploratory analysis of vibrio spp. The primary objectives of these study are to 1) detect vibrio spp. genes across samples, 2) analyze the distribution, abundance of vibrio genes across samples, 3) outline the seasonality of Vibrio spp., and 4) explore the environmental linkages of Vibrio spp

A holistic approach to understand ecological pathways of trigger and transmission of cholera is needed in order to develop prediction of conditions related to when and where the next outbreak will occur. Ecological niche modeling (ENM) offers great potential for species delimitation and distributions like Vibrio cholerae. Vibrios' present geographic distribution can be projected via ecological niche models into geographic dimensions to predict where the species will and will not be able to maintain reemergence. It utilizes associations between environmental variables and known species' location to define physical conditions (Guisan and Thuiller, 2005). So, one of the goals of this study was to generate the vibrio species distribution based on Environmental Niche Modeling (ENMs). In this study, the occurrence points for V.cholerae were 
collected from literature. Implementations of several algorithms of ENM finally resulted in the best combination of vibrio emergence that covered the entire global coast.

\subsection{Material and methods}

\subsubsection{Data sampling}

Water, oyster, and sediment samples were collected over a three year period at two locations in the Chester River $\left(39^{\circ} 05.09^{\prime} \mathrm{N}, 76^{\circ} 09.50^{\prime} \mathrm{W}\right)$ and Tangier Sound $\left(38^{\circ} 10.97^{\prime} \mathrm{N}\right.$, $75^{\circ} 57.90^{\prime} \mathrm{W}$ ) in the Chesapeake Bay, Maryland from June, 2009, to August, 2012 (Figure 5.1). During the warmer months of June through August, sampling was done twice each month and once each month during September through May. Water parameters, including water temperature, conductivity, $\mathrm{pH}$, dissolved oxygen, salinity, TDS, chlorophyll a, and turbidity measured using a secchi disk, were determined for samples collected at the surface (1ft) and bottom (1ft from the bottom) at each site and the measurements were made using a digital handheld conductivity meter (model 30-25FT; Yellow Springs Instruments, Yellow Springs, OH). 


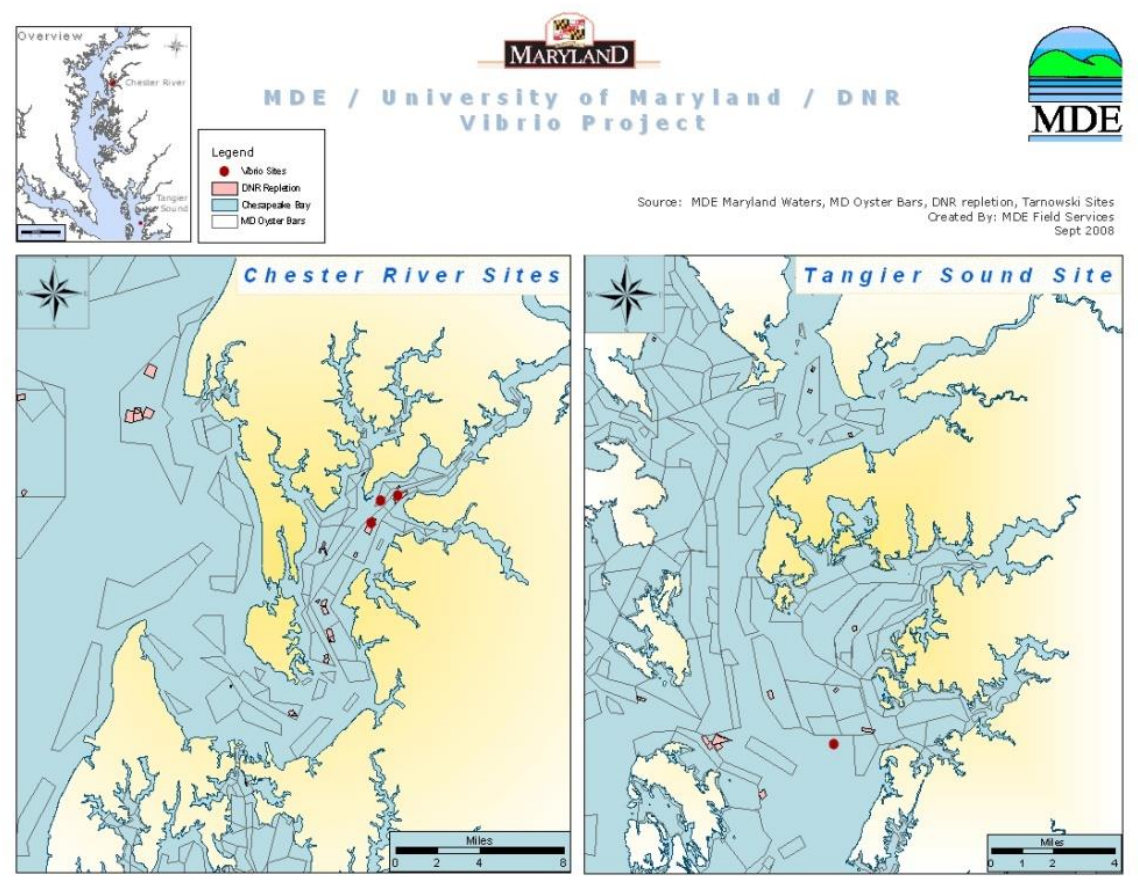

Figure 5.1. Map of Chesapeake Bay showing sampling sites in the Chester River and Tangier Sound.

In addition, air temperature, weather, wind direction, wind velocity, tide and prior rain events were also recorded. At each site, 12 liters of water, 20-25 oysters, and 80-100g of sediment (collected from oyster boxes or beds) were collected. Samples were kept on ice during transport to the laboratory at the University of Maryland, College Park and, upon arrival the samples were stored overnight at $15^{\circ} \mathrm{C}$ until processing the following morning.

\subsubsection{Water quality analysis}

Total suspended particulate matter (SPM), chlorophyll-a (chl-a), and dissolved organic carbon (DOC) samples were collected at each site. For SPM, predried GF/F filters were weighed before ca. $100 \mathrm{ml}-200 \mathrm{ml}$ of whole sample water was filtered. Filters were dried overnight at $65^{\circ} \mathrm{C}$ before being reweighed on a high precision scale. For chl-a, whole sample water volumes of between $100 \mathrm{ml}$ to $200 \mathrm{ml}$ sample water were filtered in triplicate, using $25-\mathrm{mm}$ diameter GF/F filters, and stored at $-20^{\circ} \mathrm{C}$ until analysis. Chlorophyll-a and other pigments were measured in 
methanol extracts using a Cary model 50 UV-visible-light spectrophotometer. For DOC, whole water samples were filtered using a pre-combusted GFF filter and the collected filtrate was used to sample rinse equipment. After rinsing, $100 \mathrm{ml}$ of whole water was filtered, and the filtrate was used to sample rinse three separate HDPE bottles three times. After rinsing, the HDPE bottles were filled with freshly filtered water and concentrated $\mathrm{HCl}$ was added to convert the inorganic carbon to $\mathrm{CO}_{2}$ with the bottles uncapped and left standing overnight. Samples were capped the next day and stored at $-20^{\circ} \mathrm{C}$ before measurements were taken using a Shimadzu TOC-V CSN carbon analyzer equipped with an ASI-V autosampler (Shimadzu Scientific Instruments, Columbia, MD).

\subsubsection{Vibrio presence data for proof concept}

V. cholerae at several locations in Chesapeake Bay in fall 1976 and spring 1977 was identified (Colwell et al., 1981). V. cholerae was detected in Veracruz, Mexico during April, May and June 1999 (Lizárraga-Partida, et al., 2009). Haiti documented the presence of V. cholerae and to characterize the contamination in Haitian freshwater in 2010 (Hill et al., 2011). The detection of the V. cholerae was significantly frequent at Doheny State Beach a Southern California recreational beach (Dickinson et al., 2013). V. cholerae in water, shellfish and sediment of three French Mediterranean coastal lagoons (Cantet et al., 2013). V. cholerae was in the estuarine and marine waters of the Rio de la Plata and the Argentine shelf of the Atlantic Ocean, respectively (Binsztein et al., 2004). Outcomes indicated that Vibrio cholerae, increased in dominance in North Sea, (Vezzulli et al., 2011), Germany (Frank et al., 2006), southeast Sweden (Andersson and Ekdahl, 2006).

A research on occurrence of Vibrio cholerae in Municipal and Natural Waters in Azerbaijan concluded that a distinct seasonality in cholera incidence exists in Azerbaijan in Caspian Sea and near Mingacevir reservoir (Gurbanov et al., 2012). In the Mediterranean Sea, 
vibrios have been found in three neighboring beaches along the Italian coast of the northern Adriatic Sea (Harriague et al., 2008). V. cholerae was detected at Caspian Sea in Azerbaijan (Rashid et al., 2013).

\subsubsection{Remote Sensing data used for development of framework for niche modeling}

Environmental data from sources have been used in this study but mostly satellite data have been preferred e.g. seas surface temperature data and Chlorophyll Concentration anomalies from VIIRS satellite, salinity data from Aquarius satellite have been used. International Hydrographic Organization (IHO) and Intergovernmental Oceanographic Commission (IOC) collected bathymetry data have also been used. A complete list of independent variables data are tabulated below. We have only considered the month of June to model vibrio species distribution as we found most coastal occurrence reported points in this time.

Table 5.1: Data used for coastal vibrio niche modeling

\begin{tabular}{lll}
\hline Variable & Data source & Spatial resolution \\
\hline Sea surface temperature & VIIRS satellite & $9 \mathrm{~km} \times 9 \mathrm{~km}$ \\
\hline Chlorophyll Concentration & VIIRS satellite & $9 \mathrm{~km} \times 9 \mathrm{~km}$ \\
\hline Mean salinity & Aquarius satellite & $1^{\circ} \times 1^{\circ}$ \\
\hline Bathymetry & $\begin{array}{l}\text { International Hydrographic Organization (IHO) \& } \\
\text { Intergovernmental Oceanographic Commission (IOC). }\end{array}$ & $0.25^{\circ} \times 0.25^{\circ}$ \\
\hline
\end{tabular}

\subsubsection{Statistical analysis methods}

Violin plots were used to explore the relative distribution of Vibrio genes selected for study (i.e. $t l h, t r h, t d h, v v h)$ in three sample types (water, oyster, and sediment). A violin plot combines a boxplot with kernel densities (Hintze,1998). Ternary density plots were used to capture abundance of Vibrio parahaemolyticus and Vibrio vulnificus across each sample type. Water, sediment and oyster samples were used to explore relative incidence of each of the four genes with 
respect to sample type. A ternary plot is a barycentric plot of three variables which sums to a constant and graphically depicts the ratios of the three variables as positions in an equilateral triangle.

Incidence of autochthonous Vibrio vulnificus containing $v v h$, and V. parahaemolyticus containing $t l h, t d h$, and trh at two sampling sites in the Chesapeake Bay, namely the Chester River and Tangier Sound, were depicted using individual circular seasonality plots (Barnett et al., 2012) with combined scatterplots to visualize and detect the seasonality pattern across genes. Descriptive seasonal circular plots were adopted to capture the seasonality patterns of Vibrio genes for each of the samples and sites in the Chesapeake Bay. The circular seasonal plot, commonly known as the "rose diagram" (Barnett et al., 2012), is a powerful visual tool to summarize seasonal behavior of pathogens, like $V$. vulnificus and $V$. parahaemolyticus and their annual seasonal cycles. In the circular rose diagram, the radius is proportional to the mean of the sample. Rose plots are more effective than traditional scatterplots in the context of seasonality because, for example, they place January and December values side by side, allowing for a clearer depiction of the cyclic nature of seasonal trends. The petals of the rose are representative of the mean density of vibrios in a month. Color gradient of the petals are deeper for low density improve visibility against the background.

The one way ANOVA method was used to determine if the abundance of Vibrios was different within three scenarios: a) densities differ by sample, e.g., water, oyster, and sediment; b) with respect to genes, e.g., $t l h, t d h, t r h$, and $v v h$; and c) the two sites. The method of analysis has been used widely and has been a staple of statistical modeling for many years (Stahle, 1989; Sokal, 1981). By comparing variances of the data, ANOVA examines the squares of differences, both between samples or groups, as well as between data sets within samples (Stahle, 1989). Differences 
in the mean condition the $95 \%$ confidence interval plot were employed to determine whether the densities varied with respect to the three scenarios.

Principal component analysis (Pearson,1901) was used to explore interlinkages of the two Vibrio spp. with ecohydrological variables. Principal component analysis, frequently used in biological studies (Landgrebe et al., 2002; Khan et al., 2001; Li et al., 2006; Alter et al., 2000; Holter et al., 2000) and one of its many strengths is the ability to analyze complex data efficiently without losing the original meaning of the data (Bauer and Rørvik, 2007; Dunteman, 2011; Abdi and Williams, 2010; Jolliffe, 2002). The method can be used to explain variability of data and remove factors not accounting for variation (Dunteman, 2011; Abdi and Williams, 2010; Jolliffe, 2002).

The two-dimensional principal component (PC1 and PC2) loadings plot was employed to determine correlation of the ecohydrological variables measured in this study with the incidence of genes $t l h, t d h, t r h$ and $v v h$. The magnitude of the covariation of two variables is determined by their cosine angle, $0^{\circ}-180^{\circ}$ suggests a positive correlation and $180^{\circ}-360^{\circ}$ is negative correlation.

Spatial risk modeling of pathogens is a proactive and quantitative approach (Peterson et al., 2001). The probability modeling for predictability of presence/absence (binary) of a pathogen is popularly known as species distribution model (SDM) or ecological niche model (ENM). The model utilizes associations between environmental covariates and pathogen occurrence locations to define potential risk areas for pathogenic emergence (Guisan and Thuiller., 2005). There are several algorithms under the umbrella of SDM/ENM, broadly classified as profile algorithms (e.g. bioclim, domain) and machine learning/regression algorithms (e.g. logistic regression, support vector machine, artificial neural network). The profiling algorithm computes the similarity of a location by comparing the profile of environmental variables to a percentile distribution of the 
values at known locations of occurrence. On the other hand, machine learning/ regression algorithm performs a supervised classification, where the data set is divided into two groups e.g. training and testing. The training data set is used to fit the model parameters, and the testing data set to assess the parameter estimations. Selection of a model largely depends on the type of the predictors, dependent variable, and expected outcome. We have taken a multi modeling approach and hence applied three algorithms e.g. Bioclim, Random Forest, and Support Vector Machine.

\subsection{Results and discussion}

\subsubsection{Distribution and abundance of selected Vibrio genes across samples}

A total of 54 samples were collected monthly for four years, and these yielded violin plots descriptive of the distribution of the selected Vibrio genes across samples, based on colony blot hybridization data (Figure 5.2). The Chester River (CR) and Tangier Sound (TS) sites reached Vibrio vulnificus at densities approximately two fold higher than Vibrio parahaemolyticus across all three sample types, with predominance $(\sim 71 \%)$ in water. The TS samples showed th genes were present in large numbers, as was $v v h$. The $t l h$ genes were present in high in number in the oyster ( 40\%) and sediment samples (45\%), and low in water. In contrast, $v v h$ was detected at high densities in water (66\%), compared to sediment and oysters. 

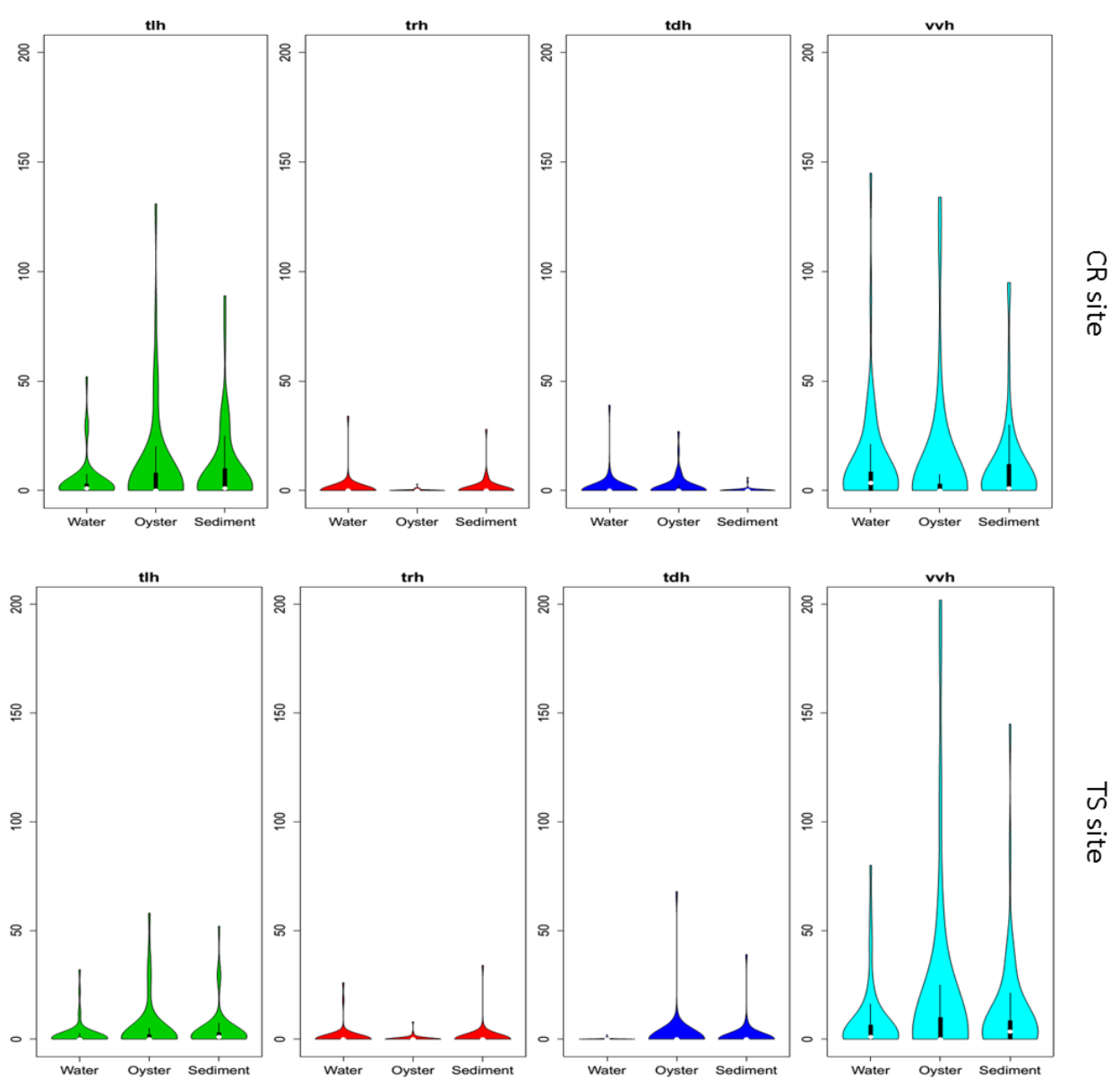

Figure 5.2. Violin plot of vibrio genes detected in water, oyster and sediment samples.

The $v v h$ gene in the CR was present at density (46\%) in oyster samples but relatively low in water (24\%) and sediment (30\%). The trh genes were detected least often (5\%) in all samples collected at both sites, notably in the both locations, especially in oyster samples. Tdh was least detected $(<2 \%)$ in water and sediment samples in both the CR and TS. Peak densities of $v v h$, trh, and $t l h$ were detected in oyster samples, i.e., $202 \mathrm{cfu} / \mathrm{gm}$ and $68 \mathrm{cfu} / \mathrm{gm}$ in $\mathrm{CR}$ samples and $131 \mathrm{cfu} / \mathrm{gm}$ in TS (Table 4). Results for the $t d h$ gene were the opposite, low count of $34 \mathrm{cfu} / \mathrm{ml}$ in TS water and sediment samples and $11 \mathrm{cfu} / \mathrm{mg}$ oyster sample in TS. 

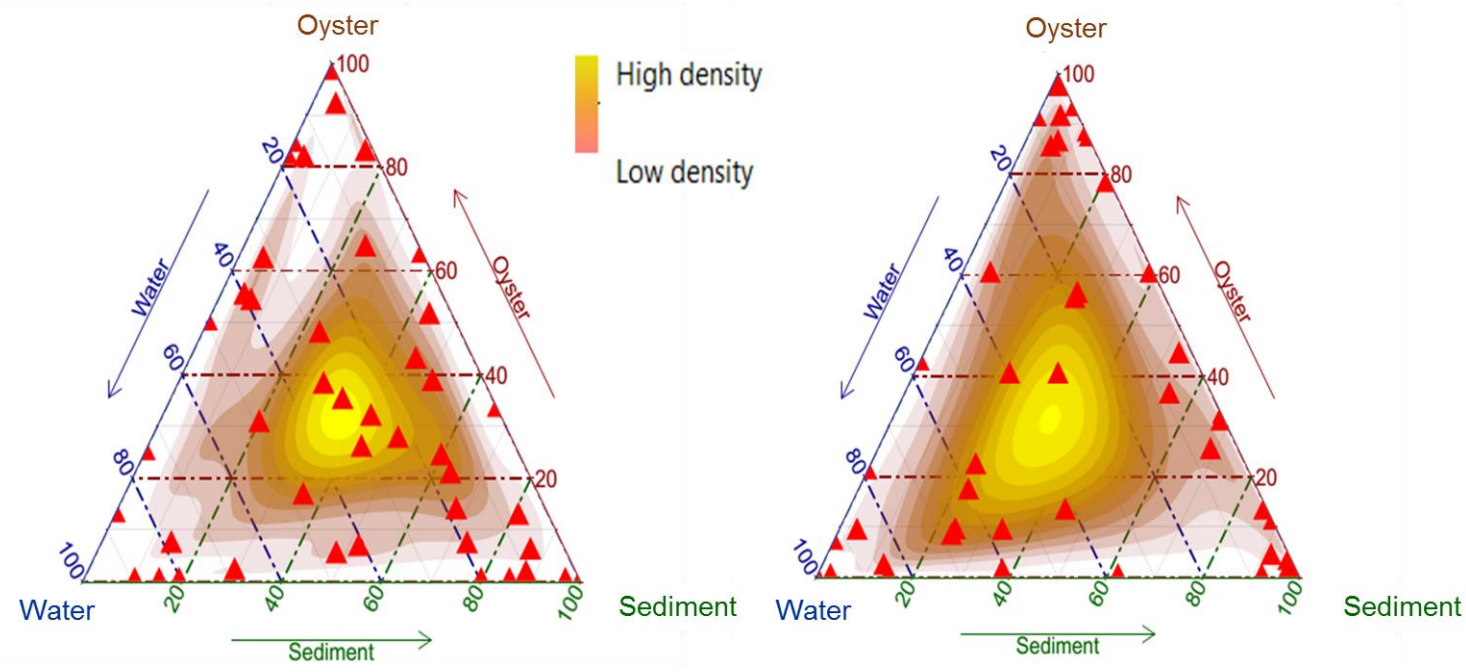

Figure 5.3. Ternary densities of Vibrio detected in water, oyster, and sediment.

The distribution of the two Vibrios spp. in samples was analyzed by plotting ternary densities, using combinations of percent Vibrio vulnificus and Vibrio parahaemolyticus in water, sediment, and oyster as the three variables (Figure 5.3). Samples from the CR yielded a triangular proportional display that indicates the CR. Oyster and sediment had high densities. This suggests the two Vibrio spp. are concentrated in oysters and sediment. In the TS, both water and sediment samples had moderate concentration of the two Vibrio spp. However, the results also indicate oysters are the main reservoir of the two Vibrio spp. in the CR and TS.

\subsubsection{Seasonality of Vibrio genes}

Articulating seasonality patterns of selected genes of the two pathogenic Vibrio spp. is important for prediction of the potential public health risk. This information provides both timing and intensity of $V$. parahaemolyticus and $V$. vulnificus and allows modeling the public health risk.

Vibrios in the $\mathrm{CR}$ are present in highest number in oyster samples during warm summer months (June to August) and in the fall of the year (September to November) (Figure 5.4). 

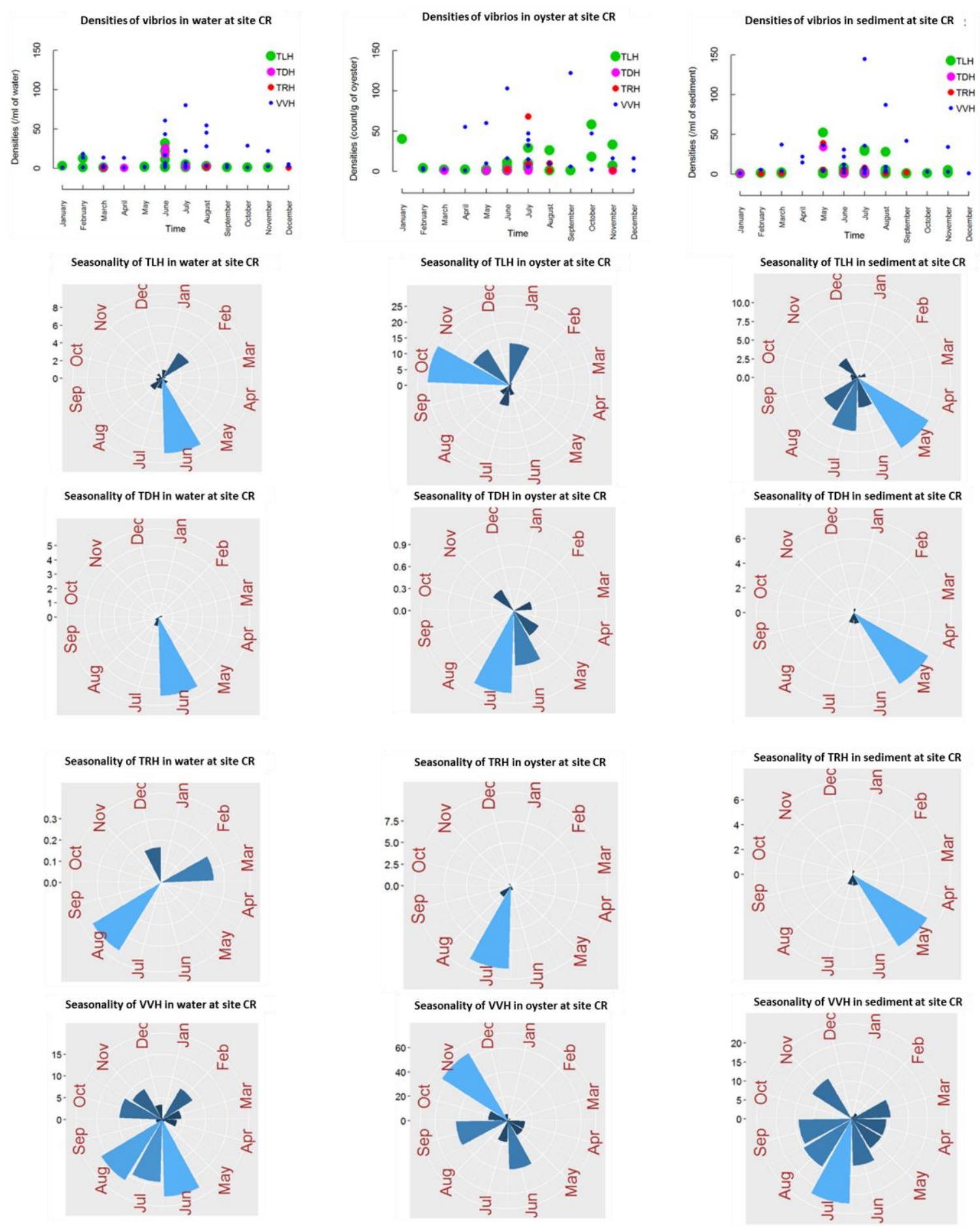

Figure 5.4. Seasonality of vibrio genes detected in water, oyster, and sediment samples. 
The $t d h$ and $t r h$ genes are detected in higher numbers during summer season, peaking in August and July respectively. In contrast, $v v h$ and $t h$ were detected in large numbers during the fall peaking in September and October, respectively.

Vibrio parahaemolyticus was detected in sediment samples in May, peaking at 34/gm, 52/gm, and 39/gm for $t d h$, th, and trh, respectively. V. vulnificus was detected at highest number in July, with $v v h$ highest at 145/gm sediment. Vibrios were detected in water samples only in the summer, with $t$ th and $t d h$ peaking in June at $32 / \mathrm{ml}$ and $26 / \mathrm{ml}$, respectively and $v v h$ present throughout summer months, with the peak density for $v v h$ in July at $80 / \mathrm{ml}$ water.

At site TS, Vibrios in oyster samples are higher in density from June to October. Most $V$. parahaemolyticus strains containing $t d h$, trh are common solely in summer, with th showing dominance in both summer and fall. The peak density for $t d h$ and $t r h$ were in July and $t d h$ in August. In contrast, $v v h$ only had significant quantities in October with a peak of 145/gm of oyster. Vibrio genes in sediment samples show variability in distribution during summer and fall. $t$ th in sediment shows abundance in these two seasons with the peak of $89 / \mathrm{mg}$ found in July. On the other hand, $t d h$ was high in June and $t r h$ in November respectively. $v v h$ are mostly abundant during early fall in September and October, with the peak density of $95 / \mathrm{mg}$ of sediment was observed in August. Unlike site CR, vibrios in water samples at site TS are only large in density during May by $V$. parahaemolyticus genes with $t d h$, th, trh peak densities of 34/ml, 52/ml and 39/ml respectively and July by $V$. vulnificus strain $(v v h)$ with a peak density of $145 / \mathrm{ml}$.

\subsubsection{Variability of Vibrio seasonality across samples, genes and locations}

To test if difference in abundance of either Vibrio vulnificus or Vibrio parahaemolyticus, in each of the three sample types, e.g., waster, oyster, and sediment by site of collection was significantly different, one way ANOVA was employed. The previous analysis 
indicated oysters had the highest number of both Vibrio spp. One way ANOVA, with Tukey's post hoc test, indicated only the Vibrio spp. in water and oysters were statistically significant ( $\mathrm{p}=0.06)$. ANOVA test results comparing CR and TS shard both sites were not significantly different.

\subsubsection{Ecological linkage of Vibrio spp. by sample type and emergence modeling}

The linkages of the Vibrio spp. and their genes with ecohydrological variables were investigated (Figure 5.5). The two-dimensional principal component (PC1 and PC2) loadings plot/ correlplot (Graffleman J. et al 2013) was employed to determine correlation of the ecohydrological variables measured in this study with the incidence of genes $t l h, t d h, t r h$, and $v v h$. The primary reasons to avoid traditional regression was the presence of collinearity within the variables. The correlation among the variables are approximated by the cosine of the angle between the two variables. The magnitude of the correlation between two variables is determined by their cosine angle, where $0^{\circ}$ $-180^{\circ}$ suggests a positive correlation and $180^{\circ}-360^{\circ}$ is negative correlation. An angle close to 0 or 360 refers to a correlation close to positive 1 versus any angle close to 90 or 270 refers a correlation close to 0 . In contrast, any angle close to 180 refers to negative 1 correlation. The additional advantage of exploring linkages using correlplot is the capacity of visualizing the variability within the variable. The length of the variable in the plot is an indication of the variability of the data where long refers to high variability and short refers to low variability. Here, all four genes show high variability in the data as the vectors are relatively long. It is important to retain correlated variables with high variability (long vector).

PC loading plots suggest water temperature, air temperature, dissolved oxygen, chlorophyll-a, salinity, and total dissolved solids are representative variables for the two Vibrio spp. with respect 
to sample type (water, sediment, and oyster) and site (CR, TS). The three V. parahaemolyticus genes were found to be closely associated with water temperature, air temperature, dissolved oxygen, and chlorophyll across samples. Specifically, $t d h$ showed the most consistent relationship with these variables across all three sample types. 

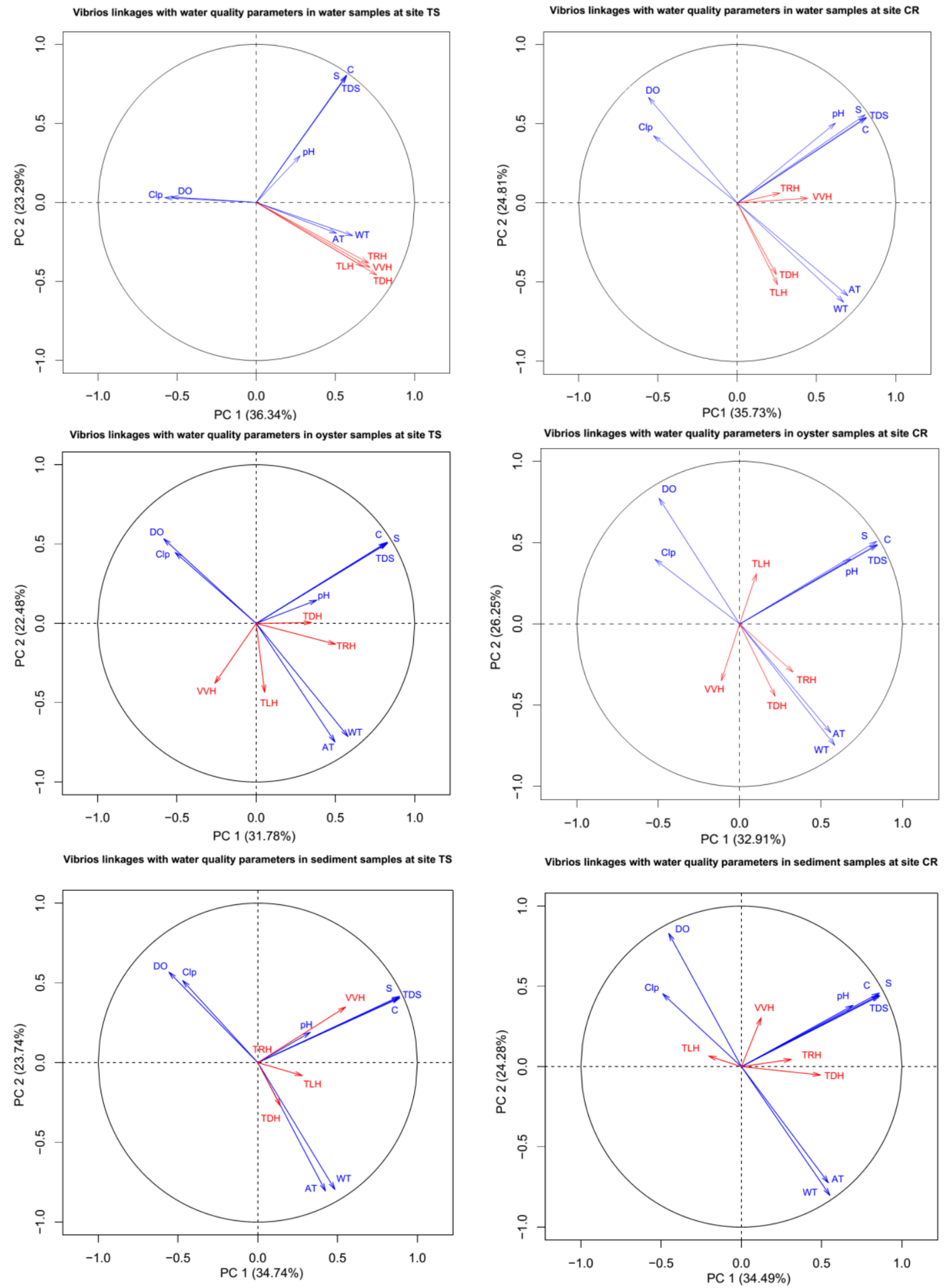

Figure 5.5. Ecological niches of vibrio genes detected in water, oyster, and sediment samples.

V. vulnificus carrying $v v h$ were associated with salinity, total dissolved solids, and $\mathrm{pH}$.

Interestingly, $V$. parahaemolyticus was positively associated with these variables, but in sediment 
samples in TS and water samples collected at the CR but negatively associated in oyster samples in the TS.

The research explores the ensemble probability distribution of vibrio growth risk in coastal water using three algorithms e.g. bioclim, random forest and support vector regression. The model was calibrated on a global scale first based on previously reported coastal occurrences and then simulated along the coast of entire globe. This study retained three algorithms (e.g. SVM, Random Forest and Bioclim) where the AUC was more than 0.5. AUC is a measure of rank-correlation. In unbiased data, a high AUC (Area Under the Curve) indicates that sites with high predicted suitability values tend to be areas of known presence and locations with lower model prediction values tend to be areas where the species is not known to be present(absent or a random point). An AUC score of 0.5 means that the model is as good as a random guess (Phillips et al., 2006). We then combined all these high performance algorithm results to generate the ensemble vibrio emergence risk on a global scale (Figure 5.6). It was also found that the Bioclim and Support Vector Machine algorithm performances were particularly useful here, as we mainly dealt with vibrio presence data from the literature and large environmental variables. 


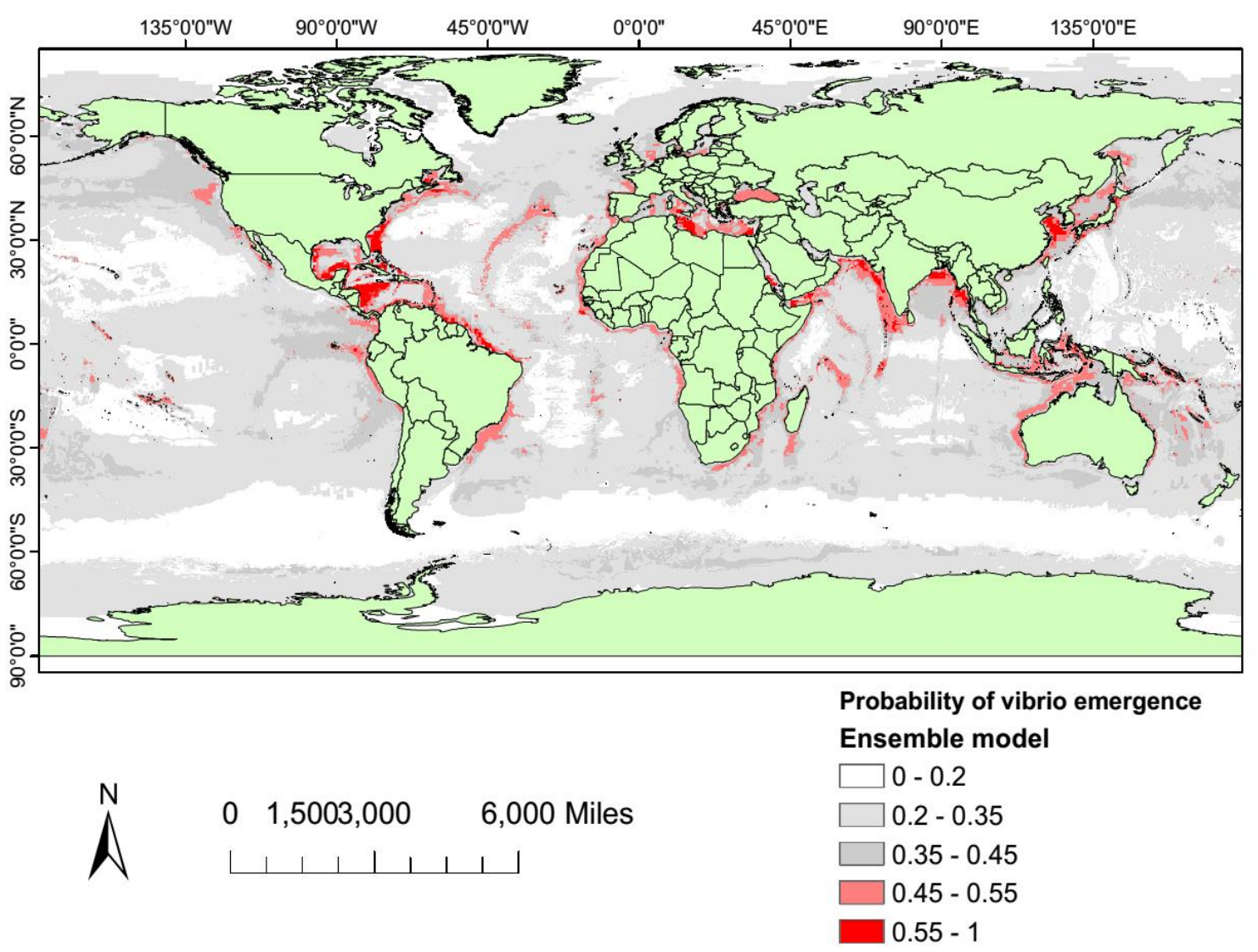

Figure 5.6: Emergence risk of V.cholerae based on ensemble model

\subsection{Conclusions}

Oysters serve as a reservoir for V. parahaemolyticus and V. vulnificus in the Chesapeake Bay. During warm months of the year, the bacterial load is high and oysters can acquire large numbers of the bacteria. An interesting discovery of this study is the seasonality observed for Vibrio vulnificus and Vibrio parahaemolyticus. In both the Chester River and Tangier Sound, the two Vibrio spp. were present in higher abundance during summer and fall months, further evidence for conclusions drawn in previous studies. Vibrio populations in oysters peaked during JuneAugust and again in September-November. $V$. vulnificus was present at peak concentrations during July and October and V. parahaemolyticus in July and August. Compared to V. parahaemolyticus, V. vulnificus was found to be present in significantly higher numbers in all sample types and at 
both sites in the Chesapeake Bay. Thus, V. vulnificus poses a more serious threat than $V$. parahaemolyticus in the Chesapeake Bay, with V. vulnificus detected in significantly higher numbers during those peak months. It is important to note that the colony blot method employed in this study is culture dependent. Therefore, the ability of all $V$. vulnificus present in the samples to grow should be taken into consideration. Complex interactions between bacteria, as well as other organisms, can impact the ability of specific species or strains to grow and proliferate. Results of this study showed that chlorophyll-a, dissolved oxygen, and water temperature were significantly associated with Vibrio spp. density and incidence of $V$. vulnificus was additionally associated with turbidity and salinity. The seasonality and abundance exploration will aid in public health risk understanding from vibrio spp. across time and samples. Exploration of the environmental linkages will guide the predictive model development for Vibrio spp. using the closely associated variables.

Primary challenge is to determine spatial and seasonal variability of risks of vibrio presence and hence abundance along the coast. Thereby, the emergence risk locations from this vibrio species modeling is supposed to decrease disease burden to offer a sustainability solution. This model results are only limited to month of June only, which should serve as a guideline for development of any future protocols of such modeling. 


\section{CHAPTER 6: CONCLUSION}

\subsection{Towards an operational near real time cholera forecasting system}

Cholera, the deadly waterborne disease is transmitted by drinking water contaminated with $V$. cholerae. It is well established that $V$. cholerae is autochthonous to aquatic habitats and proliferates in waterbodies under appropriate environmental conditions (Hasan et al., 2011) and hence cannot be eradicated from the environment. In developing countries, cholera is considered a major public health issue because of the unavailability of safe water and sanitation facilities. In the absence of a robust civil infrastructure, including safe water and sanitation, the water sources serve as point of interaction of $V$. cholerae with human populations. The disease can spread rapidly, resulting in explosive outbreaks, because of its short incubation period which ranges from few hours to 5 days after infection (WHO, 2014). Occurrence and growth of the bacteria is linked to modalities of climatic processes, and hence it is possible to develop mathematical models to determine risk of infection in human population. In this research, using satellite derived data on precipitation, temperature, population density and available water and sanitation infrastructure, it is evident that the model predicted high risk of cholera, at least four weeks in advance. The research argues that prediction of conditions conducive to cholera in human population can motivate targeted intervention efforts that must include provisions to strengthen accessibility of clean and safe water infrastructure. The hydroclimatic cholera risk monitoring system, developed in this research, is simple and yet powerful to be implemented across all cholera hotspots in the world. A proven reliable cholera prediction model will aid in public health people to reach vulnerable locations 
ahead of time with the prevention and mitigation material and equipment for cholera such as vaccines, water purification and sanitation equipment, antibiotics, oral rehydration solution (Akanda et al., 2012). With the limited efficacy of vaccines and other treatment methods, this alternated prediction device based on environmental conditions favourable for diarrheal diseases can be extremely valuable to lower the burden of global cholera.

\subsection{Development of cholera risk forecasting software (Khan Jutla cholera modeling software, V1.0) with GUI}

This study produces a R-JAVA based cholera forecasting software with user-friendly graphical user interface (GUI). The hydroclimatic risk assessment algorithm (Khan et al., 2017) is implemented and can be simulated using this software.

\section{Instructions for using the software:}

Directory:

Create three folders in directory and then download and store the following three data sets in corresponding folders

\section{Data download}

Tropical Rainfall Measuring Mission (TRMM) precipitation ( 0.25 x 0.25 degree 3B43 product resampled to 0.1 x 0.1 degree from 1998 to present) was used to estimate the historical average for precipitation. Average monthly precipitation was subtracted from the corresponding month's Global Precipitation Mission (GPM) precipitation to obtain high resolution precipitation anomalies at $0.1 \times 0.1$ degree. For air temperature anomalies, data from the National Center for Environmental Prediction (NCEP/NCAR) were used for the same time period. Population density data were obtained from Socioeconomic Data and Applications Center (SEDAC GPWv4). The location shapefiles were downloaded from DIVA-GIS server. 


\section{Current Precipitation}

https://disc.gsfc.nasa.gov/SSW/\#keywords=GPM_3IMERGDE

Historical Precipitation

https://disc.gsfc.nasa.gov/SSW/\#keywords=3B43

Temperature

https://www.esrl.noaa.gov/psd/repository/entry/show?entryid=synth\%3Ae570c8f9-ec09-4e89-

93b4-

babd5651e7a9\%3AL25jZXAucmVhbmFseXNpcy5kZXJpdmVkL3N1cmZhY2UvYWlyLm1vbi

5tZWFuLm5j

Population:

http://sedac.ciesin.columbia.edu/data/set/gpw-v4-population-density-rev10/data-download

Country of Interest

http://www.diva-gis.org/gdata

Operation of the software

$>$ Click on RStudio icon from menu and type KJCholera in the search box, located in the fourth quadrant of the interface under Packages

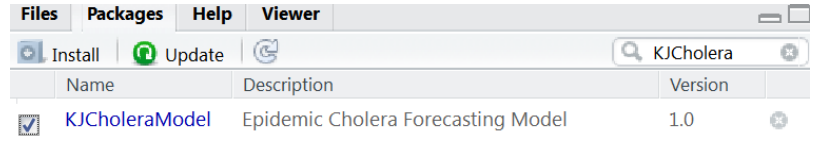

> Tick mark the KJCholeraModel and type "KJCholeraModel ()" on the third quadrant of the RStudio interface and hit enter.

$>$ It will launch the software. The software will look the picture below 


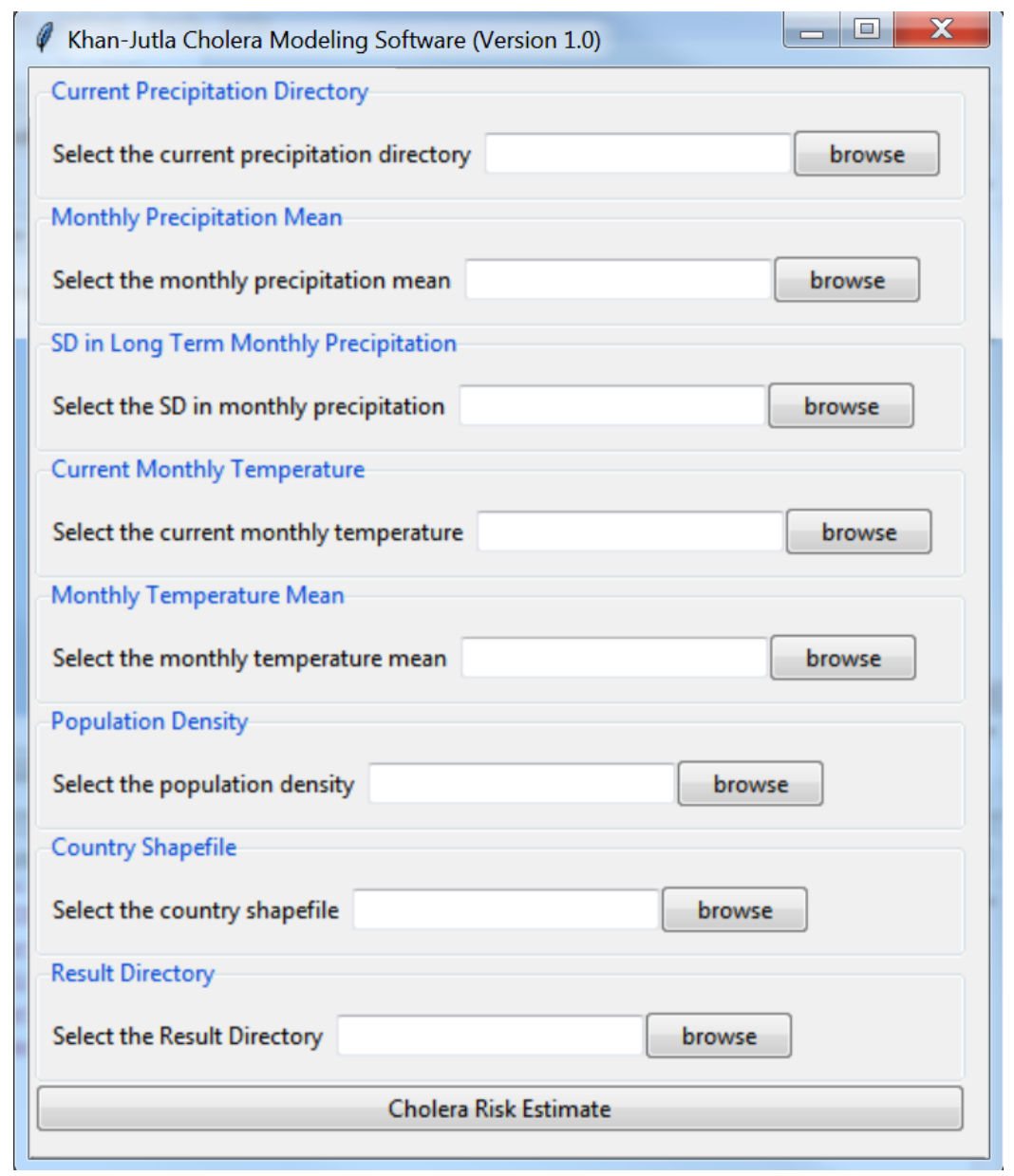

Figure 6.1 : Khan-Jutla Cholera Modeling Software (Version 1.0)

The software will ask for the following eight elements

1. Locate the folder where daily precipitations were stored (Current month)

2. Select the monthly mean precipitation for the particular month (current month)

3. Select the monthly standard deviation of precipitation for the particular month (Current month)

4. Select the current monthly temperature netcdf file (NCEP Reanalysis) (1 month lag)

5. Select the monthly mean temperature for the particular month (1 month lag)

6. Select the population density

7. Select the shapefile of the country of interest

8. Locate the folder where the results will be stored

Once done with browsing all eight elements, click on the "Cholera Risk Estimate" button. 
Check the result directory (as located in element 8) and there will be three outputs i.e. Temperature Anomaly, Precipitation Anomaly, Cholera Risk Forecast

\subsection{Operational near real time cholera forecasting in Yemen: An application}

Devastating civil unrest in Yemen has resulted in human deaths, injuries and displacement; triggering massive need for humanitarian aid. In one of the world's most water-scarce countries, the war and severe restrictions of movement of population in Yemen has made conditions worse for accessibility to clean drinking water and adequate sanitation facilities. The crisis of lack of access to safe water, sanitation and hygiene (WASH) infrastructure puts about 16 million people (UNICEF, 2017) at risk to interact with water-borne pathogens. The ongoing cholera epidemic in Yemen has already been described as the "world's worst" outbreak in history (UNICEF, 2017; WHO, 2017). As of December 2017, the human death toll stood at 2156, with a total of 815,000 suspected cases. In general, children are prime victims of this medically treatable infection (WHO, 2017). While these statistics are troublesome, the challenge remains to determine pathways to prevent infection outbreak in a human population. One of the immediate solutions is to preemptively predict environmental conditions that may lead to a cholera outbreak. Could economic and societal loss have been prevented, or at least minimized, if an early warning mechanism existed for Yemen? It is arguable that the ongoing cholera burden in Yemen could have been significantly reduced if prevention and mitigation measures had been deployed with an early warning system activated. 


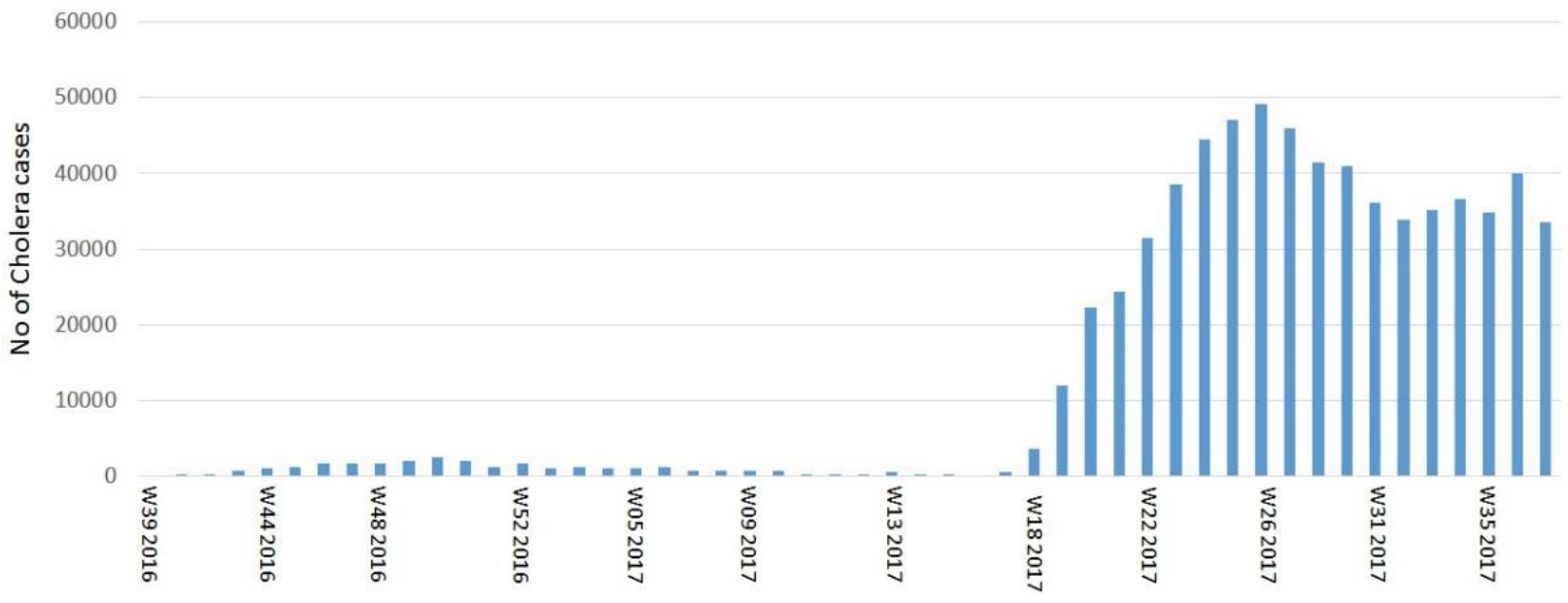

Figure 6.2: Cholera surveillance data of Yemen (WHO)

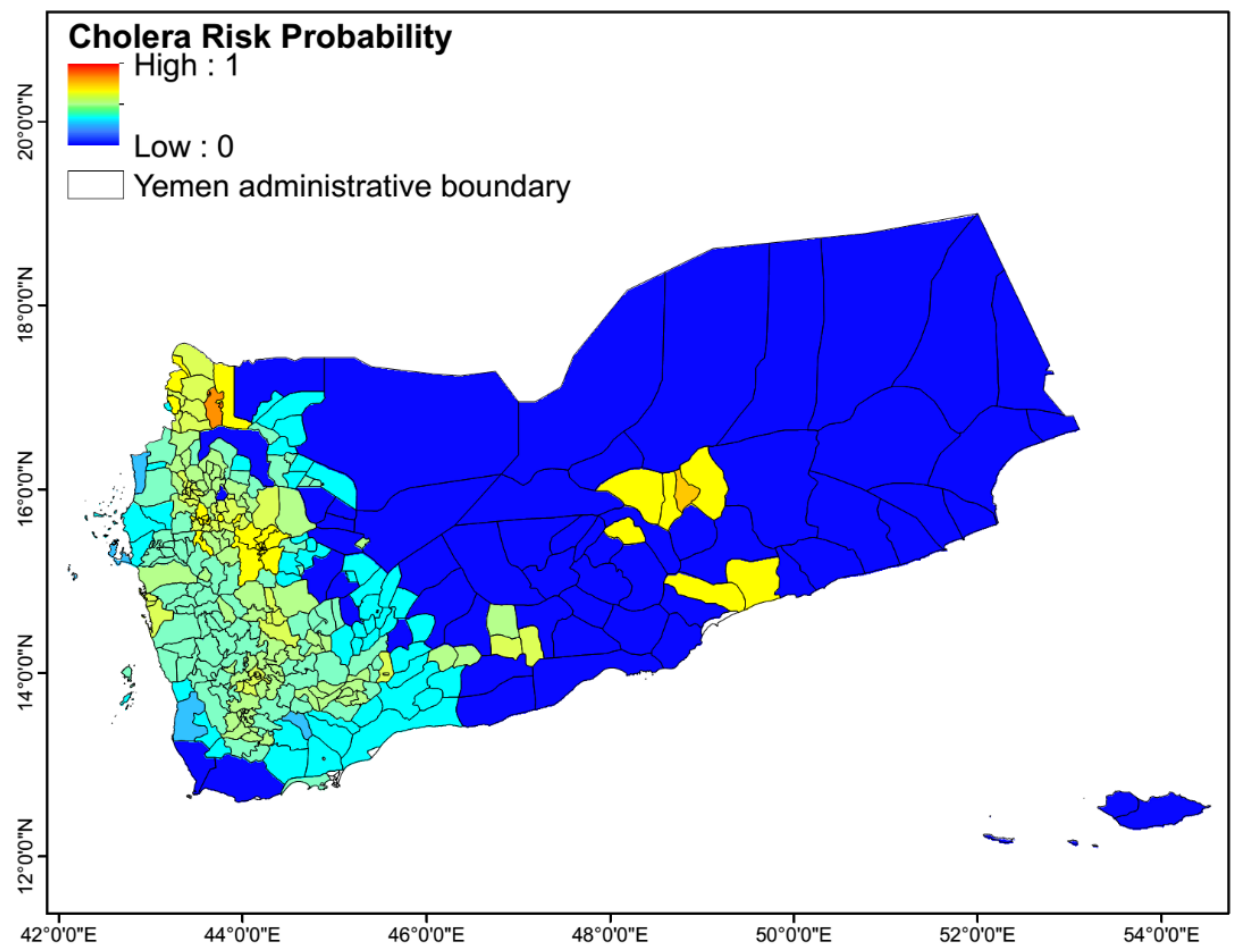

Figure 6.3: Cholera risk map for October 2016 
We prepared an October 2016 risk map using our algorithm. The model result shows there were moderate risk of a cholera outbreak in October that died out with unfavorable hydroclimatic condition starting from February 2017. The cholera risk resurged with favorable hydroclimatic condition carried by further devastated WASH facilities in April for an outbreak in May 2017. We started monitoring the hydroclimatic condition and predicting cholera risk in Yemen from March 2017 closely and prepared hydroclimatic cholera risk maps using our algorithm. By the end of April we the first hint of a cholera risk but soon after the end of May, the cholera risk prediction for June become severe in the entire eastern part of Yemen. The primary drivers behind this increased risk was a positive anomalous temperature in April was followed by a high positive anomalous precipitation in May. We had to wait until the end of June to validate this spatial cholera risk perdition with $\mathrm{WHO}$ published city level data on reported cholera patient count. The validation proved how important our cholera risk prediction map was with one month lead time.

It is highly likely that ongoing civil unrest in Yemen has placed severe stress on its already insufficient WASH infrastructure, primarily due to mass movement of the population seeking safe locations. Since the war begun in 2015, the first cholera cases showed up in October 2016 in the capital city of Sana'a and then started increasing in numbers till December but disappeared slowly. Cholera cases returned back in late April and soon started spreading with peak in June 2017. A major collapse in sewer system in early April started cross contaminating water supply and thus the drinking water. In this study, hydroclimatic conditions were monitored to determine the potential risk of cholera in Yemen beginning March 2017 (Figure 6.4a). 

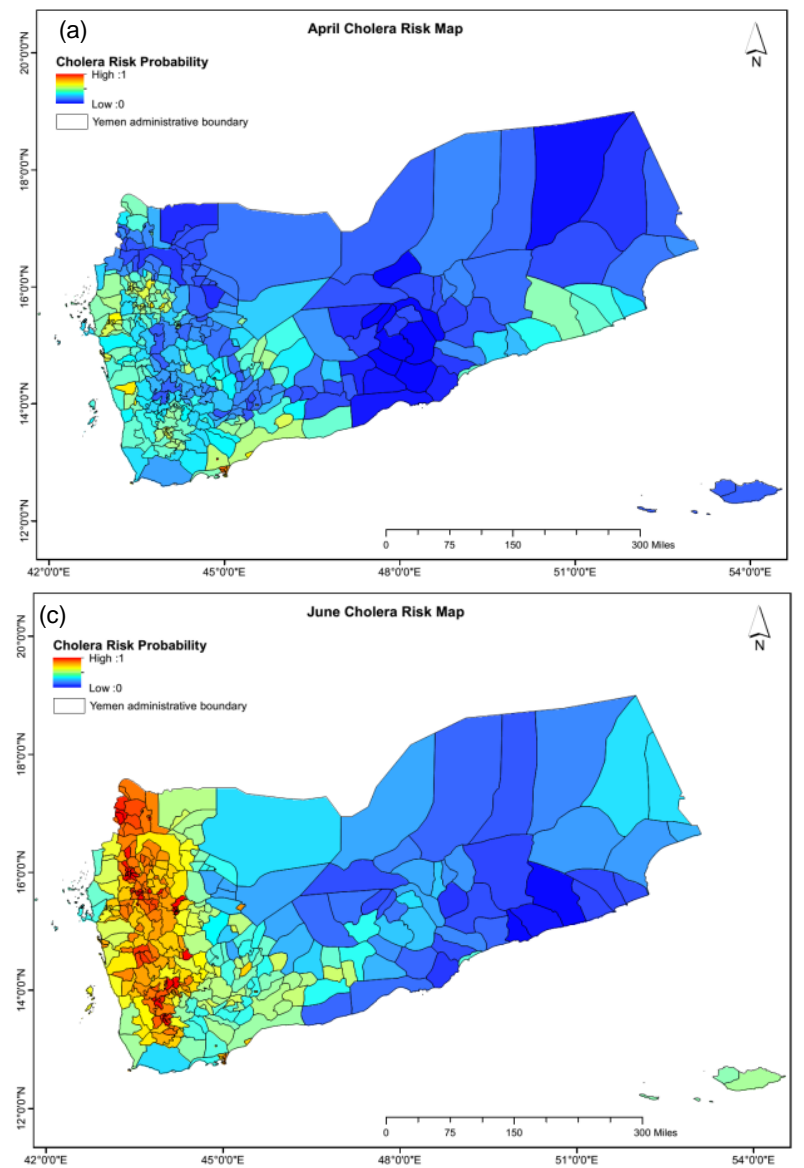

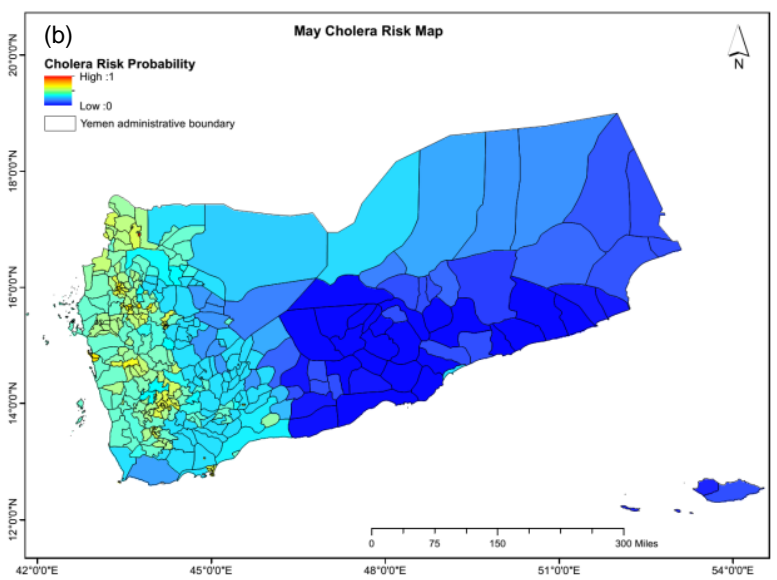

(d)

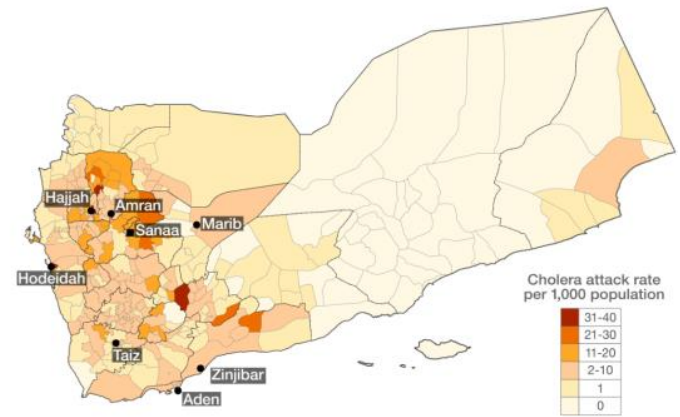

Figure 6.4: Cholera risk prediction in Yemen with one month lead time: a) April cholera risk prediction b) May cholera risk prediction c) June cholera risk prediction d) June cholera prediction validation (WHO, Aljazeera)

Correspondingly, the hydroclimatic conduciveness for cholera outbreak started anew from April and increased tremendously in May 2017. Thus, our generated cholera risk prediction maps started anticipating an outbreak beginning in April 2017. Hydroclimatic risk maps were prepared using hydroclimatic cholera risk algorithm developed in this research. At the end of May 2017, it became evident (Figure 6.4c) that the region was likely to suffer a major cholera outbreak by June 2017 , especially in western Yemen. Figure 6.4d, was obtained from WHO/ALJAZEERA, shows that an outbreak of cholera had occurred by the end of June 2017, which has a striking similarity with the 
risk map produced four weeks in advance (Figure 6.4c). The primary drivers behind the increased risk were a positive anomalous temperature in April and a positive anomalous precipitation in the following month of May. Validation of the risk map indicate robustness of our cholera prediction algorithm which has a prediction lead time of approximately one month in advance.

\subsection{Conclusion and key contributions}

Based on our cholera risk prediction across the world it is understood that prediction approach is vital for prevention and mitigation solution through proactive planning for a cholera outbreak in vulnerable regions. A continuous spatial scale cholera prediction by means of an early warning system ahead of a cholera outbreak could mean extremely vital. Spatial scale predictive modeling approach estimates the potential risk distribution probability of cholera and identifies the regions at high risk ahead of a looming outbreak and thus provide surplus time and a lessened area for establishing prevention mechanism and mitigation resource allocation to minimize the devastation the outbreak. The huge burden of the cholera outbreak globally could have been minimized, had these prediction maps were disseminated ahead of time among the end users and semi to full skilled employees involved in cholera mitigation and prevention in cholera vulnerable regions.

One of the main objectives of this research was to create a strong view on how natural disasters and cholera outbreak go hand in hand. This study aimed to contribute towards better predictability of water related infectious diseases in human population. From this context, this dissertation introduced several new components as follows

- Developed a new frameworks that assesses the short-term and long-term impact of natural disasters with response to outbreak of water sensitive infectious diseases. 
- Developed a new algorithm capable of predicting cholera outbreak risk following a water sensitive natural disaster. The algorithm was validated with realistic accuracy following two contrasting natural disasters e.g. hurricane Matthew in 2016 in Haiti, and two consecutive earthquakes in 2015 in Nepal.

- Performed a detail exploration of the distribution, seasonality and environmental linkages of vibrios in aquatic environment and modeled the global risk of coastal vibrio emergence.

- Developed a new software version (KJ Cholera Forecast, V 1.0) of the cholera algorithm with a user-friendly graphical user interface (GUI) and applied for a near real time cholera monitoring in Yemen. The software proved to be a vital tool in development of a global scale cholera warning system.

It is known that the burden of diseases like cholera can be minimized if reliable prediction can be made and relevant public health agencies alerted. Prediction systems when incorporated with vaccine protocols and long term strategies for development of civil infrastructure, can provide the capacity needed to eradicate the burden of cholera in a human population, if not the disease itself. One of the major limitations of this cholera forecast from this research is the resolution of the hydroclimatic data. The primary driver of the cholera forecast is precipitation and the resolution of a real time satellite precipitation data is $10 \mathrm{~km}$. Thereby, this cholera forecast product is more useful from the policy context at a district to state level than local village level. Interpretation of the results at a very local will be difficult unless more high resolution data were used. Moreover, the algorithm is transferable to other cholera vulnerable regions also as the precipitation risk weights are determined from the standard deviation (SD) of the data itself for the particular region of interest. Conducive conditions of temperature and other social variables (e.g. population, 
WASH) are similar across regions for triggering cholera outbreak. Thereby, the algorithm is capable of replicating cholera risk conditions across the world. Additionally, it is important to understand that $V$. cholerae grows well between $20^{\circ} \mathrm{C}$ and $37^{\circ} \mathrm{C}$ (Huq et al., 2005, Mishra et al., 2012, Constantin de Magny et al., 2011) and most cholera outbreaks were reported between $25^{0} \mathrm{C}$ and $35^{\circ} \mathrm{C}$ air temperature (Gurbanov S. et al., 2011, Jutla et al., 2011). Thereby, checking for a minimum air temperature cutoff of $20^{\circ} \mathrm{C}$ is recommended before further proceeding for a cholera risk map generation using the algorithm. Hence, above $20^{\circ} \mathrm{C}$ air temperature, extreme anomalous condition $\left.\left[>2^{0} \mathrm{C}\right)\right]$ will be considered as very high risk cholera. It is highly expected from this research that this prediction of cholera, using earth observations, especially for regions where hydroclimatic and disease surveillance data are not routinely collected, will be a critical tool to prioritize prevention and mitigation strategies, such as distribution of oral rehydration solutions, strengthening WASH infrastructure, and increasing the availability of antibiotics and vaccines. 


\section{BIBLIOGRAPHY}

Abdi H, Williams LJ., 2010. Principal component analysis. WIRE Comp Stat. 2: 433-459.

Akanda AS, Jutla AS, Gute DM, Sack RB, Alam M, Huq A, Colwell RR, Islam S., 2013. Population Vulnerability to Biannual Cholera Outbreaks and Associated Macro-Scale Drivers in the Bengal Delta. Am J Trop Med Hyg. 2013;89(5):950-959. doi:10.4269/ajtmh.12-0492.

Alam, M., Hasan, N.A., Sadique, A., Bhuiyan, N.A., Ahmed, K.U., Nusrin, S., Nair, G.B., Siddique, A.K., Sack, R.B., Sack, D.A., Huq, A., Colwell, R.R., 2006a. Seasonal Cholera Caused by Vibrio cholerae Serogroups O1 and O139 in the Coastal Aquatic Environment of Bangladesh. Appl. Environ. Microbiol. 72, 4096-4104. doi:10.1128/AEM.00066-06

Ali M, Emch M, Donnay JP, Yunus M, Sack RB. Identifying environmental risk factors for endemic cholera: a raster GIS approach. Health Place. 2002;8(3):201-210. doi:10.1016/S1353-8292(01)00043-0.

Alter O, Brown PO, Botstein D., 2000. Singular value decomposition for genome-wide expression data processing and modeling. Proc Natl Acad Sci USA. 97: 10101-10106.

Alexander, K.A., Carzolio, M., Goodin, D., Vance, E., 2013. Climate Change is Likely to Worsen the Public Health Threat of Diarrheal Disease in Botswana. Int. J. Environ. Res. Public. Health 10, 1202-1230. doi:10.3390/ijerph10041202

Andersson, N., and Mitchell, S., 2006. "Epidemiological geomatics in evaluation of mine risk education in Afghanistan: introducing population weighted raster maps.” International Journal of Health Geographics, 5, 1.

Auerbach, P. S., 2015. "Preparedness explains some differences between Haiti and Nepal's response to earthquake.” BMJ, 350(jun05 1), h3059-h3059.

Banakar, V, Constantin de Magny G, Jacobs J, Murtugudde R, Huq A, Wood RJ, Colwell RR., 2011. Temporal and spatial variability in the distribution of Vibrio vulnificus in the Chesapeake Bay: a hindcast study. Ecohealth 8, 456-467.

Barnett, AG, Baker P, Dobson AJ. 2012. Analysing Seasonal Data. The R Journal. 4/1: 5-10.

Bartlett, J.G., 2008. Infectious diseases associated with natural disasters, in: The Social Ecology of Infectious Diseases. Elsevier, pp. 351-377. 
Bartlett JG., 2016. Infectious diseases associated with natural disasters. In: The Social Ecology of Infectious Diseases. Elsevier; 2008:351-377. http://linkinghub.elsevier.com/retrieve/pii/B9780123704665500182

Baross J and Liston J. 1970. Occurrence of Vibrio parahaemolyticus and Related Hemolytic Vibrios in the Marine Environments of Washington State. Applied Microbiology. 20: 179-186.

Bartram J., 2008. Flowing away: water and health opportunities. Bull World Health Organ. 86(1):2.

Bauer, A and Rørvik, LM., 2007 A novel multiplex PCR for the identification of Vibrio parahaemolyticus, Vibrio cholerae and Vibrio vulnificus. Lett Appl Microbiol 45, 371375.

Bej, AK, Patterson DP, Brasher, CW, Jones, DD and Kaysner, CA. 1999. Detection of total and hemolysin-producing Vibrio parahaemolyticus in shellfish using multiplex PCR amplification of $t l$, tdh, and trh. Journal of Microbiological Methods 36, 2215-2225.

Beck, L. R., Lobitz, B. M., \& Wood, B. L., 2000. Remote sensing and human health: new sensors and new opportunities. Emerging infectious diseases, 6(3), 217.

Bingham, P., Verlander, N.Q., Cheal, M.J., 2004. John Snow, William Farr and the 1849 outbreak of cholera that affected London: a reworking of the data highlights the importance of the water supply. Public Health 118, 387-394. doi:10.1016/j.puhe.2004.05.007

Bompangue, D., Giraudoux, P., Handschumacher, P., Piarroux, M., Sudre, B., Ekwanzala, M., Kebela, I., Piarroux, R., 2008. Lakes as Source of Cholera Outbreaks, Democratic Republic of Congo. Emerg. Infect. Dis. 14, 798-800. doi:10.3201/eid1405.071260

Bross MH, Soch K, Morales R, Mitchell RB., 2007. Vibrio vulnificus infection: diagnosis and treatment. Am. Fam. Physician 76: 539-544.

Brown, L., Murray, V., 2013. Examining the relationship between infectious diseases and flooding in Europe: A systematic literature review and summary of possible public health interventions. Disaster Health 1, 117-127. doi:10.4161/dish.25216

Cairncross, S., Hunt, C., Boisson, S., Bostoen, K., Curtis, V., Fung, I.C., Schmidt, W.P., 2010. Water, sanitation and hygiene for the prevention of diarrhoea. Int. J. Epidemiol. 39, i193i205. doi:10.1093/ije/dyq035

Cash, R.A., Halder, S.R., Husain, M., Islam, M.S., Mallick, F.H., May, M.A., Rahman, M., Rahman, M.A., 2013. Reducing the health effect of natural hazards in Bangladesh. The Lancet 382, 2094-2103. doi:10.1016/S0140-6736(13)61948-0 
Cash BA, Rodó X, Emch M, Yunus M, Faruque ASG, Pascual M.,2014. Cholera and Shigellosis: Different Epidemiology but Similar Responses to Climate Variability. Chin W-C, ed. PLoS ONE. 9(9):e107223. doi:10.1371/journal.pone.0107223.

Centers for Disease Control and Prevention, 2016. "Vibrio Species Causing Vibriosis". Centers for Disease Control and Prevention, Web.

Chatzidaki-Livanis M, Hubbard MA, Gordon K, Harwood VJ, Wright AC., 2006. Genetic distinctions among clinical and environmental strains of Vibrio vulnificus. Appl. Environ. Microbiol. 72: 6136-6141.

Chiang, P., Musa, G., Sylk, T., Bavley, R., Keating, W., Lakew, B., Tsou, H., Hoven, C., Chiang, P., 2013. Use of GIS Mapping as a Public Health Tool\&amp;mdash;From Cholera to Cancer. Health Serv. Insights 111. doi:10.4137/HSI.S10471

Codeco, C.T., Lele, S., Pascual, M., Bouma, M., Ko, A.I., 2008. A stochastic model for ecological systems with strong nonlinear response to environmental drivers: application to two waterborne diseases. J. R. Soc. Interface 5, 247-252. doi:10.1098/rsif.2007.1135

Colombara, D.V., Cowgill, K.D., Faruque, A.S.G., 2013. Risk Factors for Severe Cholera among Children under Five in Rural and Urban Bangladesh, 2000-2008: A Hospital-Based Surveillance Study. PLoS ONE 8, e54395. doi:10.1371/journal.pone.0054395

Colwell, R.R., 1996a. Global Climate and Infectious Disease: The Cholera Paradigm. Science 274, 2025-2031. doi:10.1126/science.274.5295.2025

Colwell, R.R., 1996b. Global climate and infectious disease: the cholera paradigm. Science 274, 2025-2031.

Constantin de Magny, G., Murtugudde, R., Sapiano, M.R.P., Nizam, A., Brown, C.W., Busalacchi, A.J., Yunus, M., Nair, G.B., Gil, A.I., Lanata, C.F., Calkins, J., Manna, B., Rajendran, K., Bhattacharya, M.K., Huq, A., Sack, R.B., Colwell, R.R., 2008. Environmental signatures associated with cholera epidemics. Proc. Natl. Acad. Sci. 105, 17676-17681. doi:10.1073/pnas.0809654105

Cook DW, Bowers JC, DePaola A., 2002. Density of total and pathogenic (tdh+) Vibrio parahaemolyticus in Atlantic and Gulf coast molluscan shellfish at harvest. J. Food Prot. 65:1873-1880.

de Magny, G. C., Murtugudde, R., Sapiano, M. R., Nizam, A., Brown, C. W., Busalacchi, A. J., \& Calkins, J., 2008. Environmental signatures associated with cholera epidemics. Proceedings of the National Academy of Sciences, 105(46), 17676-17681. 
DePaola A, JL Nordstrom, JC Bowers, JG Wells, DW Cook., 2003. Seasonal abundance of total and pathogenic Vibrio parahaemolyticus in Alabama oysters. Appl. Environ. Microbiol. 69: $1521-1526$.

Drake, SL, DePaola A, Jaykus LA., 2007. An Overview of Vibrio vulnificus and Vibrio parahaemolyticus. Comprehensive Reviews in Food Science and Food Safety. 6: 120144.

Dunteman, GH., 2011. Introduction. In Principal Components Analysis. (pp. 8-15). Sage Publications, inc.

EM DAT, 2016. The OFDA/CRED International Disaster Database.

Emch, M., Feldacker, C., Yunus, M., Streatfield, P.K., DinhThiem, V., Canh, D.G., Ali, M., 2008. Local environmental predictors of cholera in Bangladesh and Vietnam. Am. J. Trop. Med. Hyg. 78, 823-832.

Enserink, M., 2010a. Haiti's Outbreak Is Latest in Cholera's New Global Assault. Science 330, 738-739. doi:10.1126/science.330.6005.738

Enserink, M., 2010b. Haiti's Outbreak Is Latest in Cholera's New Global Assault. Science 330, 738-739. doi:10.1126/science.330.6005.738

Epstein, P.R., 1993. Algal blooms in the spread and persistence of cholera. Biosystems 31, 209221.

Eppinger M, Pearson T, Koenig SSK, Pearson O, Hicks N, Agrawal S, Sanjar F, Galens K, Daugherty S, Crabtree J, Hendriksen RS, Price LB, Upadhyay BP, Shakya G, Fraser CM, Ravel J, Keim PS., 2014. Genomic Epidemiology of the Haitian Cholera Outbreak: a Single Introduction Followed by Rapid, Extensive, and Continued Spread Characterized the Onset of the Epidemic. mBio. 5(6):e01721-14. doi:10.1128/mBio.01721-14.

Faruque, S.M., Naser, I.B., Islam, M.J., Faruque, A.S.G., Ghosh, A.N., Nair, G.B., Sack, D.A., Mekalanos, J.J., 2005. Seasonal epidemics of cholera inversely correlate with the prevalence of environmental cholera phages. Proc. Natl. Acad. Sci. 102, 1702-1707. doi:10.1073/pnas.0408992102

Finger, F., Knox, A., Bertuzzo, E., Mari, L., Bompangue, D., Gatto, M., Rodriguez-Iturbe, I., Rinaldo, A., 2014. Cholera in the Lake Kivu region (DRC): Integrating remote sensing and spatially explicit epidemiological modeling. Water Resour. Res. 50, 5624-5637. doi:10.1002/2014WR015521

Fleming, G., Van der Merwe, M., \& McFerren, G., 2007. Fuzzy expert systems and GIS for 
cholera health risk prediction in southern Africa. Environmental Modelling \& Software, 22(4), 442-448.

Ford, T. E., Colwell, R. R., Rose, J. B., Morse, S. S., Rogers, D. J., \& Yates, T. L. 2009. Using satellite images of environmental changes to predict infectious disease outbreaks. Emerging infectious diseases, 15(9), 1341.

Frerichs RR, Keim PS, Barrais R, Piarroux R., 2012. Nepalese origin of cholera epidemic in Haiti. Clin Microbiol Infect. 18(6):E158-E163. doi:10.1111/j.1469-0691.2012.03841.x.

Fuller, J.A., Clasen, T., Heijnen, M., Eisenberg, J.N.S., 2014. Shared Sanitation and the Prevalence of Diarrhea in Young Children: Evidence from 51 Countries, 2001-2011. Am. J. Trop. Med. Hyg. 91, 173-180. doi:10.4269/ajtmh.13-0503

Gelting, R., Bliss, K., Patrick, M., Lockhart, G., Handzel, T., 2013. Water, Sanitation and Hygiene in Haiti: Past, Present, and Future. Am. J. Trop. Med. Hyg. 89, 665-670. doi:10.4269/ajtmh.13-0217

Goyal, M. K., Singh, V., and Meena, A. H., 2015. "Geospatial and hydrological modeling to assess hydropower potential zones and site location over rainfall dependent Inland catchment." Water Resources Management, 29(8), 2875-2894.

Graffelman, J., \& Graffelman, M. J., 2013. Package 'Correlplot'.

Griffith, D.C., Kelly-Hope, L.A., Miller, M.A., 2006. Review of reported cholera outbreaks worldwide, 1995-2005. Am. J. Trop. Med. Hyg. 75, 973-977.

Gurbanov, S., Akhmadov, R., Shamkhalova, G., Akhmadova, S., Haley, B. J., Colwell, R. R., \& Huq, A., 2011. Occurrence of Vibrio cholerae in municipal and natural waters and incidence of cholera in Azerbaijan. Ecohealth, 8(4), 468-477.

Handzel, T., Lockhart, G., Bliss, K., Gelting, R., and Patrick, M., 2013. "Water, Sanitation and Hygiene in Haiti: Past, Present, and Future." The American Journal of Tropical Medicine and Hygiene, 89(4), 665-670.

Hasan NA, Choi SY, Eppinger M, et al., 2012. Genomic diversity of 2010 Haitian cholera outbreak strains. Proc Natl Acad Sci.109(29):E2010-E2017. doi:10.1073/pnas.1207359109.

Harris, J.B., Podolsky, M.J., Bhuiyan, T.R., Chowdhury, F., Khan, A.I., LaRocque, R.C., Logvinenko, T., Kendall, J., Faruque, A.S.G., Nagler, C.R., Ryan, E.T., Qadri, F., Calderwood, S.B., 2009. Immunologic Responses to Vibrio cholerae in Patients CoInfected with Intestinal Parasites in Bangladesh. PLoS Negl. Trop. Dis. 3, e403. doi:10.1371/journal.pntd.0000403 
Hashizume, M., Armstrong, B., Hajat, S., Wagatsuma, Y., Faruque, A.S.G., Hayashi, T., Sack, D.A., 2008. The Effect of Rainfall on the Incidence of Cholera in Bangladesh: Epidemiology 19, 103-110. doi:10.1097/EDE.0b013e31815c09ea

Hashizume M, Wagatsuma Y, Hayashi T, Saha SK, Streatfield K, Yunus M. The effect of temperature on mortality in rural Bangladesh--a population-based time-series study. Int J Epidemiol. 2009;38(6):1689-1697. doi:10.1093/ije/dyn376.

Hilton T, Rosche T, Froelich B, Smith B, Oliver J., 2006. Capsular polysaccharide phase variation in Vibrio vulnificus. Appl. Environ. Microbiol. 72: 6986-6993.

Hintze, J. L., 1998. NCSS Statistical Software. Kaysville, UT: NCSS

Holter, N.S. et al., 2000. Fundamental patterns underlying gene expression profiles: simplicity from complexity. Proc. Natl. Acad. Sci. USA 97, 8409-8414.

Humanitarian bulletins, 2018. Yemen Humanitarian Bulletin Issue 30

Hunter, P. R., 2003. Climate change and waterborne and vector-borne disease. Journal of applied microbiology, 94, 37-46.

Huq A, Small EB, West PA, Huq MI, Rahman R, Colwell RR., 1983. Ecological relationships between Vibrio cholerae and planktonic crustacean copepods. Appl Environ Microbiol. 1983;45(1):275-283.

Huq, A., Sack, R.B., Nizam, A., Longini, I.M., Nair, G.B., Ali, A., Morris, J.G., Khan, M.N.H., Siddique, A.K., Yunus, M., Albert, M.J., Sack, D.A., Colwell, R.R., 2005. Critical factors influencing the occurrence of Vibrio cholerae in the environment of Bangladesh. Appl. Environ. Microbiol. 71, 4645-4654. doi:10.1128/AEM.71.8.4645-4654.2005

Iwamoto M, Ayers T, Mahon BE, Swerdlow DL., 2010. Epidemiology of seafood-associated infections in the United States.Clin. Microbiol. Rev. 23 399-411.

Johnson CN, Flowers AR, Noriea NF, III, Zimmerman AM, Bowers JC, DePaola A, Grimes DJ., 2010. Relationships between environmental factors and pathogenic vibrios in the Northern Gulf of Mexico. Appl. Environ. Microbiol.76:7076-7084.Johnson et al, 2012

Johnson, C.N., Bowers, J.C., Griffitt, K.J., Molina, V., Clostio, R.W., Pei, S., Laws, E., Paranjpye, R.N., Strom, M.S., Chen, A., Hasan, N.A., Huq, A., Noriea III, N.F., Grimes, D.J., and Colwell, R.R., 2012. Ecology of Vibrio parahaemolyticus and Vibrio vulnificus 
in the Coastal and Estuarine Waters of Louisiana, Maryland, Mississippi, and Washington (United States). Appl. Environ. Microbiol. 78(20):7249-7257.

Joh, R.I., Wang, H., Weiss, H., Weitz, J.S., 2009. Dynamics of Indirectly Transmitted Infectious Diseases with Immunological Threshold. Bull. Math. Biol. 71, 845-862. doi:10.1007/s11538-008-9384-4

Jolliffe IT., 2002. Principal Component Analysis. New York: Springer. Edition 2.

Jutla AS, Akanda, AS, Colwell R, Islam S., 2011. Warming oceans, phytoplankton, and river discharge: implications for cholera outbreaks. Am. J. Trop. Med. Hyg. 85, 303-308. doi:10.4269/ajtmh.2011.11-0181

Jutla, A., Aldaach, H., Akanda, A.S., Huq, A., Colwell, R.R., 2015. Satellite Based Assessment of Hydroclimatic Conditions Related to Cholera in Zimbabwe. PLOS-One. doi:10.1371/journal.pone.0137828

Jutla, A., Khan, R., and Colwell, R., 2017. "Natural Disasters and Cholera Outbreaks: Current Understanding and Future Outlook." Current Environmental Health Reports.

Jutla, A., Whitcombe, E., Hasan, N., Haley, B., Akanda, A., Huq, A., Alam, M., Sack, R.B., Colwell, R., 2013a. Environmental Factors Influencing Epidemic Cholera. Am. J. Trop. Med. Hyg. 89, 597-607. doi:10.4269/ajtmh.12-0721

Jutla, A.S., Akanda, A.S., Islam, S., 2013. A framework for predicting endemic cholera using satellite derived environmental determinants. Environ. Model. Softw. 47, 148-158. doi:10.1016/j.envsoft.2013.05.008

Jutla, A.S., Akanda, A.S., Islam, S., 2010. Tracking Cholera in Coastal Regions Using Satellite Observations1. JAWRA J. Am. Water Resour. Assoc. 46, 651-662. doi:10.1111/j.17521688.2010.00448.x

Kaneko, T., and R. R. Colwell., 1973. Ecology of Vibrio parahaemolyticus in the Chesapeake Bay. J. Bacteriol. 113:24-32.

Kaneko, T., and R. R. Colwell., 1975. Incidence of Vibrio parahaemolyticus in Chesapeake Bay. Appl. Microbiol. 30:251-257.

Kaneko,T., and R.R.Colwell., 1975. Adsorption of Vibrio parahaemolyticus onto chitin and copepods. Appl. Environ. Microbiol.29:269-274. 
Kaspar,C.W. and M.L. Tamplin., 1993. Effects of temperature and salinity on the survival of Vibrio vulnificus in seawater and shellfish. Appl. Environ. Microbiol. 59:2425-2429.

Kelly, MT, Stroh EM., 1988. Occurrence of Vibrionaceae in natural and cultivated oyster populations in the Pacific Northwest. Diagn. Microbiol. Infect. Dis. 9: 1-5.

Kelly, MT., 1982. Effect of temperature and salinity on Vibrio (Beneckea) vulnificus occurrence in a Gulf Coast environment. Appl. Environ. Microbiol. 44: 820-824.

Khan, J. et al. 2001. Classification and diagnostic prediction of cancers using gene expression profiling and artificial neural networks. Nat. Med. 7: 673-679.

Khan, M. U., and Shahidullah, M.,1982. "Role of water and sanitation in the incidence of cholera in refugee camps." Transactions of the Royal Society of Tropical Medicine and Hygiene, 76(3), 373-377.

Khan, R., Anwar, R., Akanda, S., McDonald, M. D., Huq, A., Jutla, A., \& Colwell, R., 2017. Assessment of Risk of Cholera in Haiti following Hurricane Matthew. The American journal of tropical medicine and hygiene, 97(3), 896-903.

Kishishita, M., N. Matsuoka, K. Kumagai, S. Yamasaki, Y. Takeda, and M. Nishibuchi., 1992. Sequence variation in the thermostable direct hemolysin related hemolysin (trh) gene of Vibrio parahaemolyticus. Appl. Environ. Microbiol. 58:2449-2457.

Koelle, K., Rodó, X., Pascual, M., Yunus, M., Mostafa, G., 2005. Refractory periods and climate forcing in cholera dynamics. Nature 436, 696-700. doi:10.1038/nature03820

Kondo, H., Seo, N., Yasuda, T., Hasizume, M., Koido, Y., Ninomiya, N., Yamamoto, Y., 2002. Post-flood--infectious diseases in Mozambique. Prehospital Disaster Med. 17, 126-133.

Kouadio, I. K., Aljunid, S., Kamigaki, T., Hammad, K., \& Oshitani, H., 2012. Infectious diseases following natural disasters: prevention and control measures. Expert review of anti-infective therapy, 10(1), 95-104.

Landgrebe J, Wurst W, Welzl G., 2002. Permutation-validated principal components analysis of microarray data. Genome Biol. 3.

Leaning, J., Guha-Sapir, D., 2013. Natural Disasters, Armed Conflict, and Public Health. N. Engl. J. Med. 369, 1836-1842. doi:10.1056/NEJMra1109877

Ligon, B. L., 2006. Infectious diseases that pose specific challenges after natural 
disasters: a review. In Seminars in pediatric infectious diseases (Vol. 17, No. 1, pp. 3645).

Li CM, Klevecz RR., 2006. A rapid genome-scale response of the transcriptional oscillator to perturbation reveals a period-doubling path to phenotypic change. Proc. Natl. Acad. Sci. USA 103: 16254-16259.

Lobitz, B., Beck, L., Huq, A., Wood, B., Fuchs, G., Faruque, A.S.G., Colwell, R., 2000. Climate and infectious disease: Use of remote sensing for detection of Vibrio cholerae by indirect measurement. Proc. Natl. Acad. Sci. 97, 1438-1443. doi:10.1073/pnas.97.4.1438

Mari, L., Bertuzzo, E., Righetto, L., Casagrandi, R., Gatto, M., Rodriguez-Iturbe, I., Rinaldo, A., 2012. On the role of human mobility in the spread of cholera epidemics: towards an epidemiological movement ecology: HUMAN MOBILITY AND CHOLERA EPIDEMICS. Ecohydrology 5, 531-540. doi:10.1002/eco.262

Masuda, N., Holme, P., 2013. Predicting and controlling infectious disease epidemics using temporal networks. F1000Prime Rep. 5. doi:10.12703/P5-6

McMichael, A., 2009. Human population health: sentinel criterion of environmental sustainability. Curr. Opin. Environ. Sustain. 1, 101-106. doi:10.1016/j.cosust.2009.07.001

Mishra, A., Taneja, N., \& Sharma, M., 2012. Environmental and epidemiological surveillance of Vibrio cholerae in a cholera-endemic region in India with freshwater environs. Journal of applied microbiology, 112(1), 225-237.

Motes, M. L., A. DePaola, D. W. Cook, J. E. Veazey, J. C. Hunsucker, W. E. Garthright, R. J. Blodgett, and S. J. Chirtel., 1998. Influence of water temperature and salinity on Vibrio vulnificus in Northern Gulf and Atlantic Coast oysters (Crassostrea virginica). Appl. Environ. Microbiol. 64:1459-1465.Newton et al, 2012.

Mukandavire, Z., Smith, D.L., Morris Jr, J.G., 2013. Cholera in Haiti: Reproductive numbers and vaccination coverage estimates. Sci. Rep. 3. doi:10.1038/srep00997

Nasrollahi, N., Kazemi, H., and Kamkar, B., 2017. "Feasibility of ley-farming system performance in a semi-arid region using spatial analysis." Ecological Indicators, 72, 239248.

Nelson, E.J., Andrews, J.R., Maples, S., Barry, M., Clemens, J.D., 2015. Is a Cholera Outbreak Preventable in Post-earthquake Nepal? PLoS Negl. Trop. Dis. 9, e0003961. doi:10.1371/journal.pntd.0003961 
Newton A, Kendall M, Vugia DJ, Henao OL, Mahon BE., 2012. Increasing rates of vibriosis in the United States, 1996-.2010: review of surveillance data from 2 systems. Clin. Infect. Dis. 54 S391-S395

Nguyen, V. D., Sreenivasan, N., Lam, et al (2014). Cholera epidemic associated with consumption of unsafe drinking water and street-vended water--Eastern Freetown, Sierra Leone, 2012. The American journal of tropical medicine and hygiene, 90(3), 518-23.

Nishibuchi M, Kaper JB. 1995. Thermostable Direct Hemolysin Gene of Vibrio parahaemolyticus: a Virulence Gene Acquired by a Marine Bacterium. Infection and Immunity. 63: 2093-2099.

Noji, E. K. (2005). Public health issues in disasters. Critical care medicine, 33(1), S29-S33.

Noji, E.K. (Ed.), 1997. The public health consequences of disasters. Oxford University Press, New York.

Nordstrom, JL, Vickery MCL, Blackstone GM, Murray SL, DePaola A, 2007. Development of a multiplex real-time PCR assay with an internal amplification control for the detection of total and pathogenic Vibrio parahaemolyticusbacteria in oysters. Appl. Environ. Microbiol. 73: 5840-5847.

Oliver, J. D. 1989. Vibrio vulnificus, p. 569-600. In M. P. Doyle (ed.), Foodborne bacterial pathogens. Marcel Dekker, Inc., New York.

Osei, F.B., Duker, A.A., Stein, A., 2012. Bayesian structured additive regression modeling of epidemic data: application to cholera. BMC Med. Res. Methodol. 12, 118. doi:10.1186/1471-2288-12-118

Pruzzo, C., Vezzulli, L., and Colwell, R.R., 2008. Global impact of Vibrio cholerae interactions with chitin. Environ Microbiol 10: 1400-1410.

Pan-American Health Organization (Ed.), 2000. Natural disasters: protecting the public`s health, Scientific Publication. Pan American Health Organization, Washington, DC.

Paranjpye, RN, Nilsson WB, Liermann M, Hilborn ED, George BJ, Li Q, Bill BD, Trainer VL, Strom MS, Sandifer PA. 2015. Environmental influences on the seasonal distribution of Vibrio parahaemolyticus in the Pacific Northwest of the USA. FEMS Microbiology Ecology. 91: 1-11.

Pascual, M., 2000. Cholera Dynamics and El Nino-Southern Oscillation. Science 289, 1766-1769. doi:10.1126/science.289.5485.1766 
Pascual, M., Bouma, M.J., Dobson, A.P., 2002. Cholera and climate: revisiting the quantitative evidence. Microbes Infect. 4, 237-245. doi:10.1016/S1286-4579(01)01533-7

Pearson, Karl., 1901. On lines and planes of closest fit to systems of points in space, Philosophical Magazine, Series 6, vol. 2, no. 11, pp. 559-572.

Penna, M.L.F., 2004. Rede neural artificial para detecção de sobremortalidade atribuível à cólera no Ceará. Rev. Saúde Pública 38, 351-357. doi:10.1590/S0034-89102004000300003

Rebaudet, S., Sudre, B., Faucher, B., Piarroux, R., 2013. Environmental determinants of cholera outbreaks in inland Africa: a systematic review of main transmission foci and propagation routes. J. Infect. Dis. 208 Suppl 1, S46-54. doi:10.1093/infdis/jit195

Reidl, J., Klose, K.E., 2002. Vibrio cholerae and cholera: out of the water and into the host. FEMS Microbiol. Rev. 26, 125-139.

Reyburn, R., Kim, D.R., Emch, M., Khatib, A., von Seidlein, L., Ali, M., 2011. Climate variability and the outbreaks of cholera in Zanzibar, East Africa: a time series analysis. Am. J. Trop. Med. Hyg. 84, 862-869. doi:10.4269/ajtmh.2011.10-0277

Rinaldo A, Rigon R, Banavar JR, Maritan A, Rodriguez-Iturbe I. Evolution and selection of river networks: Statics, dynamics, and complexity. Proc Natl Acad Sci. 2014;111(7):2417-2424. doi:10.1073/pnas.1322700111.

Rinaldo, A., Bertuzzo, E., Mari, L., Righetto, L., Blokesch, M., Gatto, M., Casagrandi, R., Murray, M., Vesenbeckh, S.M., Rodriguez-Iturbe, I., 2012. Reassessment of the 2010-2011 Haiti cholera outbreak and rainfall-driven multiseason projections. Proc. Natl. Acad. Sci. 109, 6602-6607. doi:10.1073/pnas.1203333109

Schwartz, B.S., Harris, J.B., Khan, A.I., Larocque, R.C., Sack, D.A., Malek, M.A., Faruque, A.S.G., Qadri, F., Calderwood, S.B., Luby, S.P., Ryan, E.T., 2006. Diarrheal epidemics in Dhaka, Bangladesh, during three consecutive floods: 1988, 1998, and 2004. Am. J. Trop. Med. Hyg. 74, 1067-1073.

Showstack, R. (2015). "What Can We Learn About Disaster Preparedness from Nepal's Quake?" Eos, 96.

Singleton, F.L., Attwell, R.W., Jangi, M.S., Colwell, R.R., 1982. Influence of salinity and organic nutrient concentration on survival and growth of Vibrio cholerae in aquatic microcosms. Appl. Environ. Microbiol. 43, 1080-1085.

Slayton RB, Newton AE, DePaola A, Jones JL, Mahon BE., 2014. Clam-associated vibriosis, USA, 1988-2010. Epidemiol. Infect. 142: 1083-1088.Snedecor and Cochran, 1967 
Sokal RR, Rohlf FJ. Biometry. Freeman, New York. 1981.

Stahle L, Wold S. Analysis of Variance (ANOVA)., 1989. Chemometrics and Intelligent Laboratory Systems. 6: 259-272.

Sur, D., Dutta, P., Nair, G.B., Bhattacharya, S.K., 2000. Severe cholera outbreak following floods in a northern district of West Bengal. Indian J. Med. Res. 112, 178-182.

Tamplin, M. L., and G. M. Caspers., 1992. Persistence of Vibrio vulnificus in tissues of Gulf Coast oysters, Crassostrea virginica, exposed to seawater disinfected with UV light. Appl. Environ. Microbiol. 58:1506-1510.

The Himalayan Times., 2015. "Cholera outbreak under control now'." UNICEF. (2015). UNICEF Annual Report.

UNICEF, November 2017. Yemen Humanitarian Situation Report. https://reliefweb.int/sites/reliefweb.int/files/resources/UNICEF\%20Yemen\%20Humanita rian\%20Sitrep\%20November\%202017.pdf

Vezzulli L, Pruzzo C, Huq A, Colwell RR., 2010. Environmental reservoirs of Vibrio cholerae and their role in cholera. Environmental Microbiology Reports. 2: 27-33.

Wallace BJ, Guzewich JJ, Cambridge M, Altekruse S, Morse DL., 1999. Seafood-associated disease outbreaks in New York, 1980-1994. Am. J. Prev. Med. 17: 48-54.

Waring, S. C., \& Brown, B. J. 2005. The threat of communicable diseases following natural disasters: a public health response. Disaster Management \& Response, 3(2), 41-47.

Waring, S.C., Reynolds, K.M., D’Souza, G., Arafat, R.R., 2002. Rapid assessment of household needs in the Houston area after Tropical Storm Allison. Disaster Manag. Response DMR Off. Publ. Emerg. Nurses Assoc. 3-9.

WaterAid., 2015. "WaterAid Nepal steps up efforts to prevent cholera crisis after earthquake." WaterAid.

Watson, J.T., Gayer, M., Connolly, M.A., 2007. Epidemics after Natural Disasters. Emerg. Infect. Dis. 13, 1-5. doi:10.3201/eid1301.060779

Wechsler E, D’Aleo C, Hill VA, Hopper J, Myers-Wiley D, O’Keeffe E, Jacobs J, Guido F, Huang A, Dodt SN, Rowan B, Sherman M, Greenberg A, Schneider D, Noone B, Fanella L, Williamson BR, Dinda E, Mayer M, Backer M, Agasan A, Kornstein L, Stavinsky F, Neal B, Edwards D, Haroon M, Hureley D, Cobert L, Miller J, Mojica B, Carloni E, 
Devine B, Cambridge M, Root T, Schoonmaker D, Shayegani M, Hastback E, Wallace B, Kondracki S, Smith P, Matiuck S, Pilot K, Acharya M, Wolf G, Manley W, Genese C, Brooks J, Hadler J. 1999. Outbreak of Vibrio parahaemolyticus infection associated with eating raw oysters and clams harvested from Long Island Sound - Connecticut, New Jersey, and New York., 1998. MMWR Morb. Mortal. Wkly. Rep. 48:48-51.

Wetz, JJ, Blackwood AD, Fries JS, Williams ZF, Noble RT.. 2014. Quantification of Vibrio vulnificus in an Estuarine Environment: a Multi-Year Analysis Using QPCR. Estuaries and Coasts. 37: 421-435.

WHO $\mid$ Cholera in Zimbabwe - update [WWW Document], n.d. URL http://www.who.int/csr/don/2008_12_26/en/ (accessed 10.6.14).

WHO, December 2017. Yemen Cholera Response, Weekly Epidemiological Bulletin http://www.emro.who.int/images/stories/yemen/Yemen_Cholera_Response_Weekly_Ep idemiological_Bulletin__W50_2017_Dec_11-Dec_17.pdf

Wise, BM, Gallagher NB. 1996. The process chemometrics approach to process monitoring and fault detection. Journal of Process Control. 6: 329-348.

World Health Organization. (2006). Communicable diseases following natural disasters. 1211 Geneva 27 Switzerland.

Worden, A.Z., Seidel, M., Smriga, S., Wick, A., Malfatti, F., Bartlett, D., Azam, F., 2006. Trophic regulation of Vibrio cholerae in coastal marine waters. Environ. Microbiol. 8, 21-29. doi:10.1111/j.1462-2920.2005.00863.x

World Health Organization, 2006. Communicable diseases following natural disasters (No. WHO/CDS/NTD/DCE/2006.4). 1211 Geneva 27 Switzerland.

World Meteorological Organization, 2012. Atlas of health and climate. World Meteorological Organization, Geneva.

Wright AC, et al. 1993. Rapid identification of Vibrio vulnificus on nonselective media with an alkaline phosphatase-labeled oligonucleotide probe. Appl. Environ. Microbiol. 59: 541546.

Wright AC, Hill RT, Johnson JA, Roghman MC, Colwell RR, Morris JG Jr. 1996. Distribution of Vibrio vulnificus in the Chesapeake Bay. Appl. Environ. Microbiol. 62: 717-724.

Wutich, A., Ragsdale, K., 2008. Water insecurity and emotional distress: coping with supply, 
access, and seasonal variability of water in a Bolivian squatter settlement. Soc. Sci. Med. 1982 67, 2116-2125. doi:10.1016/j.socscimed.2008.09.042

Zuckerman, J.N., Rombo, L., Fisch, A., 2007. The true burden and risk of cholera: implications for prevention and control. Lancet Infect. Dis. 7, 521-530. doi:10.1016/S14733099(07)70138-X

Dear Dr. Studholm,

Thanks for your email. I am really glad to learn about Princeton Climate Analytics and application of hydrologic data to efforts eradicating Malaria will be a very exciting area to work.

I am eagerly waiting to join the team at Princeton but the arrival date is dependent on my work authorization (OPT) approval. I am hoping to get the OPT approval by late November.

Regards

Rakib 